\title{
ENTRE CONEJOS, DIABLOS Y VENDEDORES DE CACA: RASGOS GRAMATICALES DEL OLUTECO EN TRES DE SUS CUENTOS ${ }^{1}$
}

ROBERTO ZAVALA

\section{Introducción}

El oluteco, también conocido en la literatura como popoluca de Oluta, es una lengua de la familia mixezoque. Actualmente se hablan doce de las trece lenguas conocidas de esta familia (Wichmani 1995). El oluteco forma parte de la rama mixeana junto con cuatro lenguas del subgrupo mixeano, además del sayulteco (popoluca de Sayula), y del tapachulteco (extinto). El oluteco se habla en Oluta, municipio de Oluta, Veracruz. Los olutecos se refieren a su lengua con el nombre de ya:k?auni que deriva de ya:k 'nativo' y ?aw 'boca'. Existen aproximadamente dos decenas de personas que tienen al oluteco como primera lengua y que la hablan con fluidez, todos ellos mayores de sesenta años. Existe un igual número de "semihablantes", es decir, personas que recuerdan parte del léxico, algunas expresiones que les permite tener breves interacciones, además de que son capaces de entender parcialmente la lengua hablada por los hablantes más competentes. También existen un gran número de "recordadores", es decir, personas que conocen un reducido paradigma deexpresiones y que tienen un léxico muy limitado pero que no entienden el idioma producido por los hablantes competentes. ${ }^{2}$

\footnotetext{
${ }^{1}$ El presente trabajo es el resultado parcial de un amplio proyecto que tiene como fin la documentación del oluteco. Las diversas etapas de esta investigación que inicié en el verano de 1994 han sido financiadas por la Universidad de Guadalajara, la Universidad Nacional Autónoma de México, Conacyt (1994-1995), National Geographic Society (\#5319-94), National Science Foundation (SBR-9411247), y el Max Planck Institute for Psycholinguistics. Estoy profundamente agradecido con los siguientes hablantes del oluteco quienes han colaborado y demostrado inmensa generosidad durante los diversos períodos que he pasado en el campo: Antonio Asistente, †Lorenzo Molina, Rafaela Santander, †Jesús de los Santos, Inez Díaz, Nicolasa de los Santos, Josefa de los Santos, Otilio de Dios, †Victor González, Alfredina Asistente, Ruperta Pérez, †Mario Melchor, Bonifacio Canuto, Tomás de los Santos, †Bartolo Flor, †Agripino Molina, $†$ Claudio Pavón y †Criserio Molina. Muchas gracias también a Heriberto Avelino, Colette Grinevald, Barbara Villanova y Verónica Vázquez por sus comentarios a este trabajo.

${ }^{2}$ Sobre la clasificación entre las distintas categorías de hablantes en el caso de lenguas minoritarias o en situaciones de muerte de lenguas véase L. Campbell, y M. Muntzel 1989, y C. Grinevald, 1997.
} 
Aparte de la lista de palabras publicadas por el médico Calderón (1908), reproducidas también por Lehmann (1920), el único estudio lingüistico del oluteco es el diccionario bilingüe de Clark (1981) que contiene un vocabulario de aproximadamente dos mil entradas además de un apéndice con paradigmas verbales, y un esbozo de la fonología y de la morfología. Hasta el presente no se ha publicado ningún texto ni tampoco existe ninguna gramática de esta lengua en vías de extinción.

Los dos especialistas que se han ocupado recientemente del estudio comparativo de las lenguas mixezoques consideran al oluteco como la lengua más conservadora de esta familia debido a que mantiene rasgos fonológicos y morfológicos ausentes en otros miembros de la familia y que han sido reconstruidos como rasgos del ancestro común de las lenguas mixezoqueanas (Wichman 1995, Kaufman 1995).

Las tres narraciones que analizo a continuación forman parte de una amplia colección de textos y conversaciones de diversos géneros (recopiladas en más de cien horas de grabación con formato en video y/o magnetofónico) que he recogido, analizado, y transcrito con varios hablantes de oluteco. Las cuentos de "El conejo que quería crecer", "El diablo al que emborracharon" y "Los vendedores de caca", muy típicos del área mesoamericana, fueron narrados por el señor Antonio Asistente Maldonado de aproximadamente setenta años. El Sr. Asistente Maldonado es un excelente hablante debido a que usó el idioma materno cotidianamente hasta 1994, año en que murió su padre a una edad de ciento tres años. El Sr. Asistente ha sido mi principal colaborador, en el estudio del léxico, de la gramática y del discurso oluteco.

\section{Convenciones de transcripción y glosado}

Los tres textos analizados están transcritos siguiendo una ortografía práctica utilizada por el Proyecto para la Documentación de Lenguas de Mesoamérica (PDLMA) dirigido por T. Kaufman, J. Justeson y R. Zavala. Ia única desviación con respecto a la ortografia utilizada por el PDLMA es la forma en que se transcribe el cierre glotal. Mientras que el PDLMA usa el símbolo 7 para representar el cierre glotal, en este trabajo yo utilizo el símbolo fonético sugerido por IPA $/ 2 /$ Los otros símbolos en esta ortografia práctica son: $\ddot{u}=/ \partial /, x=/ \mathrm{s} / \mathrm{tz}=/ \mathrm{ts} /, \mathrm{ch}=/ \mathrm{tg} /, \mathrm{j}=/ \mathrm{h} /$, $\pi=/ \tilde{r} /$ y la cantidad vocálica que se representa con la vocal seguida por los dos puntos $N: /$.

La primera línea de los textos es la representación fonológica. La segunda línea es la representación morfofonológica donde el guión "-" separa morfemas, el signo de igual " =" separa clíticos y el signo de adición " + " une morfemas que forman unidades semánticas no composicionales, ya sea porque una de las partes es un elemento 
sincrónicamente no productivo o porque el significado de las partes no predice de forma composicional el significado del compuesto morfológico. Los guiones bajos " _" en la tercera línea se usan para unir al conjunto de palabras que en la glosa del español tienen correspondencia con un sólo morfema oluteco.

Al igual que muchas otras lenguas mesoamericanas, el oluteco no tiene las mismas categorías semánticas ni gramaticales del español. El uso de infinitivos para las glosas verbales, al igual que las marcas de género y número gramatical asociadas a los sustantivos de las glosas del español no se deben de interpretar como correspondencias uno a uno con los morfemas del oluteco. El oluteco no cuenta ni con infinitivos, ni con género, ni con número gramatical obligatorio.

\section{Un esbozo gramatical}

En este apartado el lector encontrará una descripción tanto de los rasgos gramaticales más frecuentes en los textos como de los rasgos estructurales del idioma que son más interesantes desde el punto de vista tipológico. La descripción no pretende ser exhaustiva debido a las limitaciones de espacio. Este esbozo es tan sólo una guía para que el lector interesado en el estudio de la morfosintaxis de las lenguas mixezoqueanas y de las lenguas mesoaméricanas en general pueda entender los diferentes tipos de estructuras morfológicas y sintácticas que se encuentran en los textos.

\section{GENERALIDADES}

El oluteco es una lengua que marca los argumentos centrales en el núcleo predicativo (Nichols 1986). Es una lengua ergativa e inversa. El núcleo verbal, siendo una palabra polimorfémica, contiene información sobre los argumentos centrales de la oración, aspecto, modo, negación, direccionales, movimiento asociado, reflexivo, incrementadores de la valencia (aplicativos y causativos) y reductores de valencia (pasivos), así como nombres y adverbios incorporados. La lengua distingue dos tipos de oraciones: las independientes y las dependientes. Las oraciones dependientes son estructuras que siguen a verbos matrices, auxiliares y adverbios. Los dos tipos de oraciones pueden identificarse debido a que presentan distintos patrones de marcación de persona, aspecto e inverso. 
MARCADORES DE ASPECTO

La lengua cuenta con dos paradigmas de aspecto, uno que aparece en oraciones independientes y otro en oraciones dependientes. En cada paradigma se pueden reconocer tres aspectos: incompletivo, completivo e irreal (Cuadro 1).

CUADRO 1: DOS PARADIGIAAS DEMARCAS DEASPECTO

INDEPENDIENTE

DEPENDIENTE

INCOMPLETIVO

$-p a$ (intransitivo)

$-i /-e^{3}$

-pe (transitivo)

COMPLETIVO

$-u$

IRREAL

-am (directo)

$-i$

an...pa (inverso) -a?n-e (inverso)

$-a ? n$ (directo)

\section{MARCADORES DE PERSONA}

Los argumentos centrales de la oración (agente, paciente, benefactivo y los otros argumentos que se suman a la valencia verbal por medio de aplicativos) no necesitan ser expresados por nominales o pronombres externos al predicado. Los argumentos centrales se expresan de manera explícita en el verbo por medio de marcadores de persona, marcas de plural, o se pueden inferir por el tipo de marcación inversa. Existen tres diferentes juegos de clíticos de persona a los que me refiero como: Juego A, Juego B y Juego C. ${ }^{4}$ En la lengua se distinguen tres personas en el singular y cuatro

${ }^{3}$ Las bases verbales cuya última sílaba tiene alguna de las vocales /a, e, o/ seleccionan al sufijo -e en las conjugaciones incompletivas dependientes. En estas mismas conjugaciones las bases verbales cuya última sillaba tiene alguna de las vocales $h, \ddot{u}$, u/ seleccionan al sufijo $-i$. Esto es evidencia de que el sufijo homófono - $i$ de las conjugaciones completivas dependientes es un morfema distinto.

${ }^{4}$ Las siguientes son las abreviaturas utilizadas para glosar las categorias gramaticales de los textos: 1 = primera persona; 2 = segunda persona; $3=$ tercera persona; $A=$ marcador de persona del Juego A (ergativo de independientes, absolutivo de dependientes y posesivo); $\mathrm{ABS}=$ absolutivo; $\mathrm{ADVZR}=$ adverbializador; $\mathrm{AN}=$ clítico para animados; $\mathrm{APL1}=$ aplicativo dativo de interés; $\mathrm{APL} 2=$ aplicativo benefactivo; $\mathrm{APL} 3=$ aplicativo asociativo y comitativo; APL4 = aplicativo instrumental; $\mathrm{AUMENT}=$ aumentativo; $\mathrm{B}=$ marcador de persona del Juego $\mathrm{B}$ (absolutivo de independientes); $\mathrm{C}=$ marcador de persona del Juego $\mathrm{C}$ (ergativo de dependientes y marcador local); CAUS = causativo; $\mathrm{CFCT}=$ contrafactual; $\mathrm{CNCT}=$ conector; $\mathrm{COMD}=$ completivo de dependientes; $\mathrm{COMI}=$ completivo de independientes; $\mathrm{COND}=$ condicional; $\mathrm{DEF}=$ definido; $\mathrm{DESID}=$ desiderativo; $\mathrm{DIM}=$ diminutivo; $\mathrm{DIR}=$ direccional $\mathrm{DIST}=$ distal, $\mathrm{DRC}=$ directo; $\mathrm{ERG}=$ ergativo; $\mathrm{EV}=$ evidencial; $\mathrm{EXCL}=$ exclusivo; $\mathrm{EXCLAM}=$ morfema exclamativo; IMPR = imperativo; INCD = incompletivo de dependientes; INCI.I = incompletivo de independientes intransitivo; INCIT = incompletivo de independientes transitivo; INTENS = intensificador, $I N V=$ inverso; INVD.C = inverso de dependientes en completivo; INVD.I = inverso de depedientes en 
en el plural. El paradigma de cuatro personas en el plural se forma con los mismos proclíticos del singular y con sufijos plurales. La vocal del sufijo plural para primera y segunda persona es una copia de la vocal del morfema que aparece antes del sufijo -Vt. El sufijo-Pütz marca primera persona de plural exclusiva [III-183]. ${ }^{5}$ El sufijo -küxx aparece de manera optativa en los verbos donde un argumento central es tercera persona [cf. I-21 vs I-18]. Los tres juegos de persona se presentan a continuación en el Cuadro 2:

\begin{tabular}{|c|c|c|c|c|c|c|}
\hline \multirow[b]{2}{*}{$\mathrm{P}$} & \multicolumn{6}{|c|}{ CUADRO 2. PARADIGMASDE MARCASDE PERSONA 6} \\
\hline & & Juego A & Juego $B$ & Juego C & Plural & EXCL \\
\hline E & 1 & $\tan =$ & $t a=$ & $\operatorname{tax}=$ & $-V t$ & -?ütz \\
\hline $\mathrm{R}$ & & & & & & \\
\hline$S$ & 2 & $\min =$ & $m i=$ & $\operatorname{mix}=$ & $-V t$ & \\
\hline O & & & & & & \\
\hline $\mathrm{N}$ & 3 & $2 i=$ & $\phi=7$ & $t a=$ & $-k u ̈ u x$ & \\
\hline
\end{tabular}

En oraciones independientes los proclíticos de los Juegos A o B preceden al predicado. El Juego A tiene una distribución ergativa: agente de transitivo [I-1]. El Juego $B$ tiene una distribución absolutiva: el único participante de predicados monovalentes $[I-4, I-6]$, paciente de bivalentes agentivos $[I-8]$, segundo participante

incompletivo; IRRD = irrealis de dependientes; IRRI = irrealis de independientes; LOC = locativo; MIRAT $=$ mirativo; NEG = negación; NMZR $=$ nominalizador; NMZR_INSTR $=$ nominalizador instrumental; $\mathrm{PAH}=$ participante del acto de habla; $\mathrm{PASV}=$ pasivo; $\mathrm{PCP}=$ participio; $\mathrm{PERF}=$ perfectivo; PL = plural de sustantivos; PL.PAH = plural para participantes del acto de habla; PL3 = plural para tercera persona; $\mathrm{POSD}=$ poseedor; $\mathrm{PROX}=$ proximal; $\mathrm{PUNTL}=$ puntual; $\mathrm{RECIP}=$ recíproco; REPET = repetitivo; RFLX = reflexivo; RLTVZR = relativizador; $S U P E R F=$ parte del cuerpo que refiere a una superficie; TEMP = posposición temporal; TERM = terminativo.

${ }^{5}$ Los ejemplos se citan de la siguiente manera: Los números romanos I, II, III identifican a una de las tres narraciones, mientras que los números cardinales identifican las oraciones específicas dentro de una de las tres narraciones donde se encuentra ejemplicado el rasgo que se discute en el artículo. Por ejemplo, cuando me refiero a la oración número dos del primer texto "El conejo que quería crecer" únicamente lo cito como [1-2]. Los ejemplos que no provienen de ninguno de los tres textos se citan con números cardinales entre corchetes.

${ }^{6}$ Algunos hablantes (entre ellos los que consultó Clark) usan la vocal /ï/ en el paradigma de los marcadores de persona A1, B1, C1 y C3 que aparecen en el cuadro con la vocal baja /a/. Hay evidencia comparativa que sugiere que las formas con $/ \mathrm{a} /$ son innovaciones mientras que las formas con /ii/ son similares a las protoformas reconstruidas para el protomixeano (Wichmann 1995:95100).

${ }^{7}$ La marca de absolutivo $\varnothing=$ para tercera persona no se representa en las glosas de los textos. 
(locativo o experimentante) de predicados bivalentes no agentivos [1-89], y tercer participante (benefactivo, meta) de verbos trivalentes [III-216]. El Juego A marca también al poseedor de los sustantivos [I-171].

El segundo tipo de marcación para aspecto y persona ocurre en oraciones dependientes (después de adverbios, auxiliares y verbos matrices). Las oraciones dependientes seleccionan las marcas de aspecto a partir de la columna de la derecha del Cuadro 1. En este tipo de oraciones, el Juego $C$ tiene una distribución ergativa: agente de transitivo [1-6]. El Juego A tiene una distribución absolutiva: el único participante de predicados monovalentes [I-160], paciente de bivalentes agentivos [I20], segundo participante (locativo o experimentante) de predicados bivalentes no agentivos [I-178], y tercer participante (benefactivo o meta) de verbos trivalentes [III$185]$.

A partir de la distribución de los marcadores de persona es claro que el oluteco sigue un patrón ergativo-absolutivo. El Cuadro 3 resume el tipo de oraciones en las cuales los tres juegos de persona ocurren y las funciones de cada juego. Este tipo de distribución de marcadores de persona, donde el ergativo de oraciones independientes funciona como absolutivo de oraciones dependientes, además de el hecho de que hay un segundo juego ergativo para oraciones dependientes, no se ha documentado en la literatura de lenguas ergativas (Dixon 1994).

$\begin{array}{lll} & \text { CUADRO 3. JUEGOS DE PERSONA } & \\ & \text { TIPO DE ORACIÓN: } & \\ & \text { Independiente } & \text { Dependiente } \\ \text { FINCIÓN: } & & \\ \text { Ergativo } & \mathrm{A} & \mathrm{C} \\ \text { Absolutivo } & \mathrm{B} & \mathrm{A}\end{array}$

PATRONES DE MARCACIÓN DIRECTO E INVERSO

Las oraciones con predicados bivalentes o trivalentes del oluteco presentan dos tipos de marcación conocidos como los patrones directos e inversos en la literatura de lenguas algonquinas (Klaiman 1992. Hockett 1966), lenguas tanoanas, (Klaiman 1989, 1992, 1993) maasai (Payne, et. al. 1994), tupi-guaraní (Payne 1994), huasteco (Zavala 1994), chinanteco de Sochiapan (Foris 1993) entre otras. ${ }^{8}$ En el patrón directo

\footnotetext{
${ }^{8}$ La alternacia entre las oraciones directas vs inversas también se encuentra en las lenguas de la rama mixeana (Wichmann 1995:90-101). El sistema inverso del mixe de Tlahuiltoltepec to discuten W. Foley y R. D. Van Valin (1985:288-291) a partir de los datos de Lyon 1967. Ninguno de los tres últimos autores hace explícito que se trata de un sistema inverso.
} 
la marca de persona en el verbo es ergativa y refiere al agente. En el caso de los verbos transitivos y bitransitivos el patrón directo ocurre cuando el primer argumento seleccionado por el verbo (agente) es más alto en la jerarquía de prominencia que el segundo argumento (paciente) [II-23] o tercer argumento (benefactivo) [III-175] seleccionado por el verbo. Es decir, una oración es directa cuando el agente es primera o segunda persona, y el paciente o benefactivo es tercera persona. La oración también es directa cuando el agente es una tercera persona más alta en animacidad o prominencia discursiva que el paciente $[\mathrm{I}-60]$ o benefactivo [I-132] que es también tercera persona (3:3). En el patrón inverso la marca de persona en el verbo es absolutiva y refiere al paciente $o$ al benefactivo. La construcción inversa ocurre cuando las condiciones se revierten, es decir, cuando el argumento paciente [I-18] o benefactivo [III-68] es más alto en la jerarquía de prominencia que el agente (3:3). El verbo que sigue el patrón directo en aspecto incompletivo toma el sufijo -pe, propio de los verbos transitivos [1-72]. Por otra parte, el verbo que sigue el patrón inverso en aspecto incompletivo toma el sufijo - $p a$, propio de los verbos intransitivos [1-8]. El patrón inverso requiere del sufijo inverso - $\ddot{u}$ en independientes [I-8], o del sufijo $-j$ [I20] ("inverso incompletivo") o $-y$ ("inverso completivo") [I-33] en dependientes. $\mathrm{El}$ patrón inverso en oluteco no es un proceso de detransitivización ya que todos los participantes seleccionados por el verbo tienen características de argumentos centrales, es decir, no es el caso de que el agente pase a ser un argumento "demovido" como en el caso de la pasiva, donde el agente no puede expresarse en la oración ni siquiera como un argumento oblicuo [1-64]. Las construcciones reflexivas [1-73] y reciprocas [I-191] se expresan siguiendo el patrón inverso. Las construcciones donde tanto el agente como el paciente son participantes del acto de habla (PAH), es decir, primera o segunda persona, siguen otro patrón que en la literatura de lenguas algonquinas se le conoce como local (Hockett 1966). En oluteco 1:2 (primera persona actuando sobre segunda persona) es una construcción local directa [1-30], mientras que 2:1 (segunda persona actuando sobre primera persona) es una construcción local inversa [I-15]. El marcador de persona para todas las construcciones locales tanto independientes como dependientes es el proclítico tax $=$ del Juego $C$. El sufijo -(V)k es la marca de inverso para la construcción local 2:1 [1-30]. El verbo que sigue el patrón local en aspecto incompletivo toma el sufijo $-p a$, propio de los verbos intransitivos [I-106, I-161]. El Cuadro 4 resume la distribución de los patrones directo e inverso en el paradigma de verbos transitivos. 
CUADRO 4. PATRONES DIRECTO E INERSO CONVERBOS TRANSITIVOS.

\begin{tabular}{ccccl}
\multicolumn{5}{c}{ NO-AGENTE (Paciente/Benefactivo) } \\
A & \multicolumn{1}{c}{1} & 2 & \\
G & 1 & INV(RFLX) & LOCAL.DRC & DRC \\
E & & & & \\
N & 2 & LOCAL.INV & INV(RFLX). & DRC \\
T & & & & \\
E & 3 & INV & INV & $\operatorname{INV(RFLX)~}$ \\
& & & & $\operatorname{DRC}\left(3: 3^{\prime}\right) / \operatorname{INV}\left(3^{\prime}: 3\right)$
\end{tabular}

Las oraciones que tienen a un agente de tercera persona y a un paciente 0 benefactivo de primera o segunda persona del plural pueden seguir el patrón inverso esbozado arriba, o pueden seguir un patrón alternante que es exclusivo a este tipo de combinación de argumentos. En el segundo patrón, que formalmente no es inverso, el agente se marca con absolutivo mientras que el paciente o benefactivo no se marca directamente con marcas de persona en el verbo. El morfema - Pit "PAH" se sufija a los verbos que siguen este patrón alternante [III-126].

La oposición directo/inverso no sólo aparece con verbos transitivos canónicos sino también con predicados bivalentes no agentivos como Pit 'existir', jo:y 'carecer', Put 'gustar', jotan 'estar enojado' y muchos más. Con este tipo de predicados la construcción directa se manifiesta cuando la locación o el experimentante es más bajo en la jerarquía de prominencia que el tema, por ejemplo, cuando el tema es primera persona y el locativo o experimentante es tercera persona [III-10]. El patrón inverso ocurre cuando las condiciones se revierten, es decir, cuando el locativo es más prominente que el tema [III-23]. En el patrón directo el locativo se expresa como un argumento oblicuo, con adposición, mientras que en la construcción inversa los dos participantes se codifican como argumentos centrales. Algunos predicados bivalentes no agentivos presentan únicamente el patrón inverso. Dentro de este grupo se encuentran verbos que no pueden expresar el tema como argumento sintáctico externo al predicado. El tema se expresa como un argumento incorporado (kayan-?o:m 'comida-estar_de_antojo') o está lexicalizado dentro de la semántica verbal [II-75, III-16]. Los verbos que en su forma no derivada tienen una estructura argumental diferente a la de los verbos bivalentes no agentivos pueden derivarse con un aplicativo o un pasivo y adquirir la estructura argumental semejante a la de los verbos bivalentes no agentivos. Existen dos tipos de verbos derivados con una estructura argumental semejante a la de los verbos bivalentes no agentivos: los 
intransitivos con el aplicativo küj- que introduce un benefactivo, ${ }^{9} \mathrm{y}$ los pasivos de los verbos ditransitivos [1-220, II-43, III-214]. Es claro que estos dos tipos de verbos derivados no son agentivos y siempre aparecen siguiendo el patrón inverso debido a que el participante más prominente en topicalidad o agentividad no coincide con el primer argumento seleccionado por el verbo, en este caso el tema.

\section{AFIJOS Y CLITICOS EN EL VERBO}

Las raíces verbales en el oluteco coocurren con clíticos, afijos y otras raíces nominales, verbales y adverbiales. Hay algunos clíticos (posiciones 16, 17, 18) que aparecen tanto con verbos como con sustantivos. He podido identificar siete posiciones que preceden a la raíz verbal y diecinueve posiciones que le siguen. Las posiciones se identifican con números cardinales que son seguidos o precedidos por guiones (afijos) o signos de igual (clíticos). Los números menores representan las posiciones más cercanas a la raíz verbal, mientras que los números mayores representan las posiciones más separadas de la ráz verbal:

$7=\quad$ Persona (Juegos $\mathrm{A}, \mathrm{B}, \mathrm{C}$ )

$6=$ Negación $(k a:=)[\mathrm{I}-72, \mathrm{I}-87, \mathrm{I}-109, \mathrm{III}-9, \mathrm{III}-146]$

$5=\quad$ Mirativo $(j a:=)[1-87, \mathrm{I}-111, \mathrm{III}-175]^{10}$

4- Pasivo (vak-) [I-33, I-172, III-191] y Causativo (yak) $[\mathrm{I}-72, \mathrm{I}-221, \mathrm{II}-29, \mathrm{III}-105]$

3- Aplicativos (küj; toj- [I-195, III-14, III-17, III-88], mü:- [I-18, I-29, I-50, I-205, III192])

2- Reflexivo (ni-) [1-73] y Recíproco (ni-) [II-29, II-45]

1- Sustantivos incorporados [II-105, III-9, III-17, III-105], morfemas de partes del cuerpo [III-108, III-226] y adverbios incorporados [I-40]

RAÍZ VERBAL (simple [I-1], compleja [I-39, III-164, III-226] o nominalizada [I-19, II-109])

$-1 \quad$ Direccionales [I-42, I-51, I-198, III-14, III-78, III-142]

-2 Desiderativo (-Po:k) [I-19, I-39] y Terminativo (-ka?) [II-109]

-3 . Puntual (-ti:y?) [I-40, I-41, I-139, I-140, II-40, II-65]

4 Plural para tercera persona $(-k \ddot{u} x)$ [I-21, I-154, II-3, II-60]

-5 Aplicativo benefactivo (-ay, a2x, -a?) [I-6, I-132, I-190, III-43]

-6 Repetitivo (-pow) [III-169, III-201]

-7 "ya" (-nii) [I-36, I-134, II-15, II-61; II-75, III-242]

${ }^{9}$ Este tipo de verbos derivados no ocurre en ninguno de los tres textos analizados pero el ejempo (6) más abajo ilustra este tipo de construcción.

${ }^{10}$ La categoría morfológica de mirativo, también conocida como admirativo, ocurre en los verbos para indicar que la información es sorpresiva o inesperada (DeLancey 1997). 
8 Inverso de independientes (-ü) [I-8, I-21, II-45, II-75, III-43], inverso en irreal (-an-ï) $[1-50$, III-214] marcador de participante del acto de habla $(-P i t)$ [III126]

$-9 \quad$ Aspecto de independientes ( $\mathrm{pa}[\mathrm{I}-3],-\mathrm{pe}[\mathrm{I}-13],-u ?-\mathrm{w}[\mathrm{I}-46, \mathrm{II}-44]$, - am [I1]), modal imperativo (-a [1II-207], $-\ddot{u}[$ [I-58], - ta [III-177]), aspecto de dependientes $(-i 2=-e[\mathrm{I}-6, \mathrm{I}-20, \mathrm{I}-23, \mathrm{I}-180],-i[\mathrm{I}-11, \mathrm{I}-23],-a$ in $[\mathrm{I}-15, \mathrm{I}-34, \mathrm{I}-$ 47]), participio $(-V k)[$ II- 63, II-112, II-240]

$-10 \quad$ Inverso dependientes $(-j[\mathrm{I}-20],-y[1-33, \mathrm{I}-67])$

-11 Peffectivo (-2a?) [II-44, III-43, III-226]

-12 Inverso local $(-(V) k)[-15,1-22,1-58]$

-13 Plual para participantes de acto de habla (-(V):t) $[$ [I-151, II-27, III-177, III-182]

-14 Exclusivo (-Pütz) [III-182]

$=15$ "también" (= Pampok) [III-35]

$=16$ "todavía" (=na) [I-58, III-152]

$=17$ Evidencial reportativo $(=x i i)[1-3,1-37, \mathrm{II}-46]$

$=18$ "nomás" $(=k 0 j)[1-37, \mathrm{I}-135, \mathrm{II}-44]$

$=19$ Tercem persona animado $(=(a) k,=a ?)[\mathrm{I}-3, \mathrm{I}-6, \mathrm{I}-18, \mathrm{I}-19]$

El verbo de las oraciones imperativas afirmativas se sufija con la marca de imperativo $(-\ddot{u} /-a)$ y no recibe los proclíticos de persona [1-58, III-132]. En la negación del modo imperativo, el verbo se sufija con la marca de incompletivo para dependientes $(-i /-e)$ en lugar del sufijo imperativo. En este tipo de oración el proclítico de negación precede al proclítico de persona [III-83], lo cual contrasta con el orden de estos proclíticos en las oraciones no imperativas donde el proclítico de persona precede al de negación [III-198].

\section{AFIIOS Y CĹTICOS EN EL SUSTANTIVO}

La morfología nominal es muy simple en comparación con la morfología verbal. Los enclíticos en quinta, sexta y séptima posición aparecen tanto en la morfología verbal como en la nominal. El templete que muestra el orden de afijos y clíticos en el sustantivo es el siguiente:

$2=\quad$ Posesivo (Juego A) $[1-87, \mathrm{I}-171$, III-203]

1. $\quad$ "otro" (ja:-) [II-30, III-129], "ajeno" (jay-) [II-85]

RAÍZ NOMINAL

-1 Diminutivo (-(?u)nak) [I-1, III-23], (-(2a)wok) [III-149], aumentativo

$(-n a ? a w)[1-131]$

$2 \quad$ Plual (-tük) [II-21, III-59] 


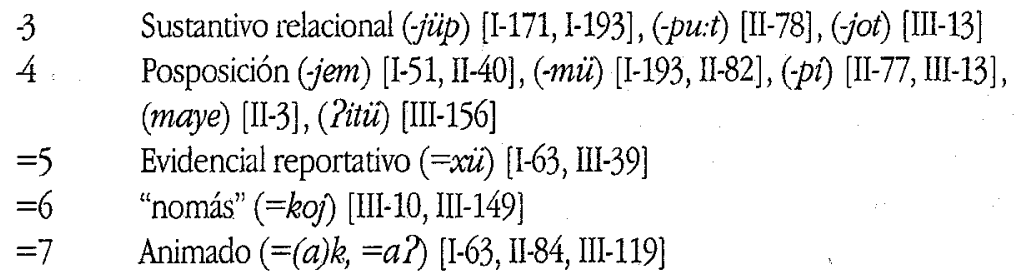

\section{COMPUESTOS VERBALES Y PROCESOS DE GRAMATICALIZACIÓN}

El oluteco permite que dos o más de dos raíces verbales aparezcan dentro de una base verbal modificada por un mismo juego de clíticos y afijos. El agrupamiento de raíces verbales para formar palabras nuevas es un proceso medianamente productivo. El significado de estos compuestos en algunos casos puede ser predecible a nivel composicional [II-9, III-226] pero en otros casos, alguno de los elementos del compuesto ya no es productivo sincrónicamente [II-12] y solamente se puede inferir que fue una raíz verbal cuando se compara con etimologías que se han reconstruido a partir del estudio de otras lenguas de la familia mixe-zoque (Wichmann 1995). Algunos de los verbos de los compuestos verbales se han gramaticalizado y han pasado a ser afijos derivativos. En los textos analizados se encuentran varios de estos afijos gramaticalizados a partir de raíces verbales:

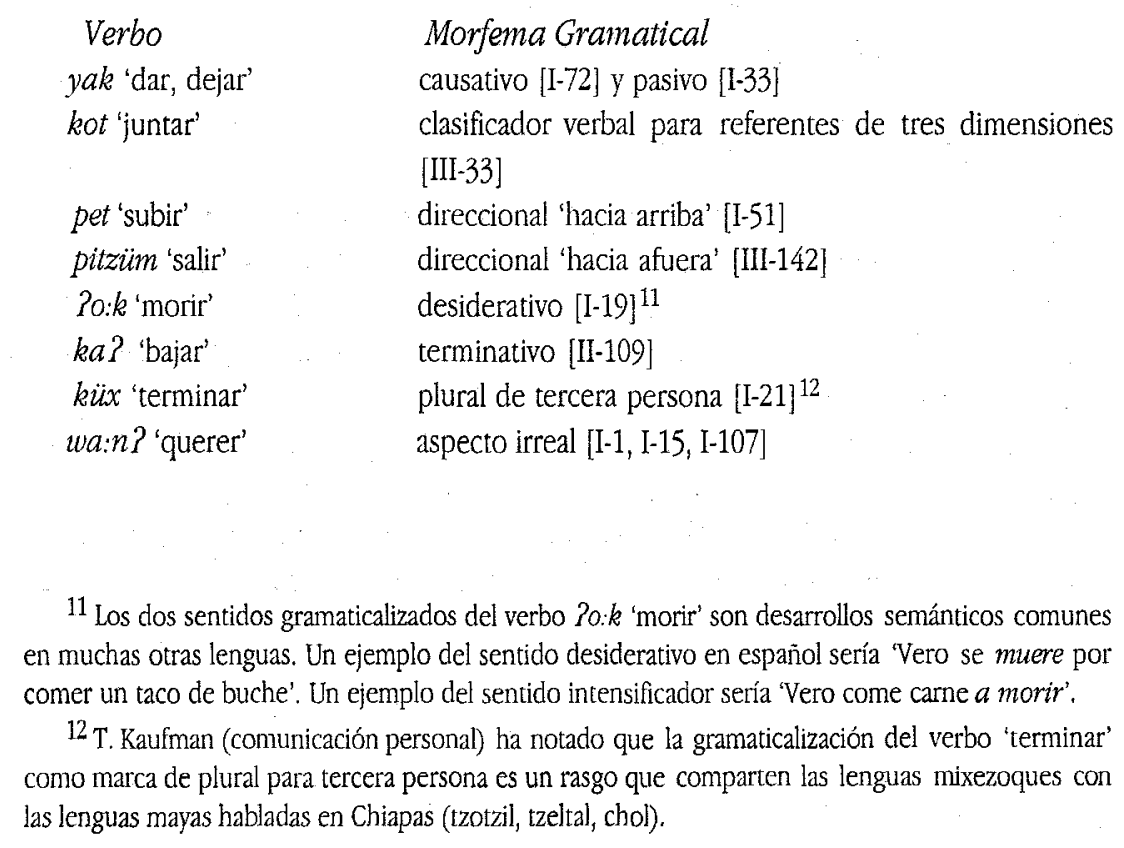

\footnotetext{
${ }^{11}$ Los dos sentidos gramaticalizados del verbo $20: k$ 'morir' son desarrollos semánticos comunes en muchas otras lenguas. Un ejemplo del sentido desiderativo en español sería 'Vero se muere por comer un taco de buche'. Un ejemplo del sentido intensificador serfa 'Vero come carne a morir'.

${ }^{12} \mathrm{~T}$. Kaufman (conunicación personal) ha notado que la gramaticalización del verbo 'terminar' como marca de plural para tercera persona es un rasgo que comparten las lenguas mixezoques con las lenguas mayas habladas en Chiapas (tzotzil, tzeltal, chol).
} 
Otras raíces verbales gramaticalizadas ausentes en los textos analizados son las siguientes: wakx 'clasificador verbal para referentes de dos dimensiones' (derivado del verbo 'extender') (1), Po:k 'intensificador' (derivado del verbo 'morir') (2) y un grupo amplio de verbos de movimiento (nükx 'ir', mi:n? 'venir', Poy 'ir y regresar', jamat 'llegar a otro lugar', ya?t 'llegar aquí', wit 'andar' wimpit 'regresar', tük Pi:y? 'entrar', nax 'pasar', tuk 'cruzar', tij 'quedar') que han pasado a ser direccionales (en la misma posición que pet [1-51] y pitzüm [III-142]) y marcadores de movimiento asociado ' $\mathrm{X}$ realiza una acción simultánea a la acción de moverse en cierta dirección' (Wilkins 1991) cuando van precedidos por el conector tak- (3) y (4). ${ }^{13}$

(1) je? ?u:ra tan=ni-piw-wakx-i-j-i:t-?ütz

esa hora Al(ABS)=RECIP-recoger-extender-INCD-INVD.I-PL.PAH-EXCL

A esa bora nos dispersamos.

(2) ta=wit-?o:k-nü-w

$\mathrm{B} 1(\mathrm{ABS})=$ andar-INTENS-ya-COMI

Ya caminé mucbo.

(3) mix=to:k-tak-wit-a?n yowa-mo:k

$\mathrm{C} 2(\mathrm{ERG})=$ vender-CNCT-andar-IRRD joven-maíz

Vas a andar vendiendo elotes.

(4) porke jamaj=ak ?i=kay-tak-nükx-küx-pe kuytüm

porque aquel=AN $\mathrm{A} 3(\mathrm{ERG})=$ comer-CNCT-ir-3PL-INCI.T aguacate

Porque ellos van (en su camino) comiendo aguacate.

\section{AUMENTO Y REDUCCIÓN DE LA VALENCIA VERBAL}

El oluteco cuenta con siete afijos que cambian la valencia verbal. En los textos analizados se encuentran oraciones con el morfema yak- marcador de pasivo [I-33], y causativo [I-72]. El pasivo yak-reduce la valencia verbal de los verbos transitivos. Los pasivos olutecos [I-172, III-191] son pasivos impersonales ya que en estas estructuras el agente no puede expresarse ni siquiera como un argumento oblicuo.

En los textos también aparecen los aplicativos toj- 'instrumental' [1-195], mü:'asociativo, comitativo' [1-18], y -ay, a?x, -a? que son los tres alomorfos del 'benefactivo' $[1-6]$. Los otros tres prefijos que aumentan la valencia verbal y no aparecen en los textos son: el causativo ta: $k$ - que se combina con verbos agentivos

13 La caregoría gramatical de "movimiento asociado" no se había identificado en lenguas mesoamericanas. 
(5), el dativo de interés küj- que se combina con verbos intransitivos (6), y el asociativo de sintagmas nominales tomo $(7) .14$

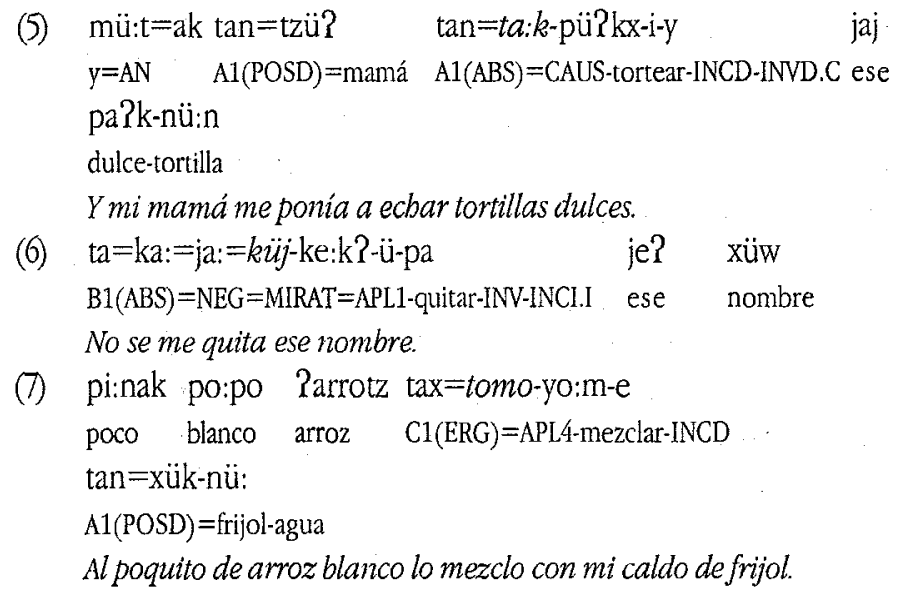

Los aplicativos aumentan la valencia verbal, de tal manera que los verbos monovalentes pasan a ser bivalentes y los verbos bivalentes pasan a ser trivalentes. Los aplicativos toj-, mü̈-, tomo- hacen posible que argumentos periféricos ocupen la posición de argumentos centrales. Los morfemas aplicativos - $a y, a P x$, $a$ ? permiten que el benefactivo se codifique como objeto primario de la oración (Dryer 1986). Mientras que el aplicativo kijj- "promueve" al benefactivo de oraciones intransitivas a la posición de un segundo argumento central que tiene las mismas propiedades de los segundos argumentos de los predicados bivalentes no agentivos que se discutieron arriba.

\section{INCORPORACIÓN DE SUSTANTNOS Y ADVERBIOS}

El oluteco es una lengua que incorpora adverbios [I-40] y sustantivos [II-105, III-9, III17, III-105]. Los morfemas incorporados preceden a la raíz verbal y le siguen varios proclíticos. Los adverbios que más frecuentemente se incorporan son: we $2 x$ 'con suerte', jane 'de a mentiras', jutztüki 'otra vez' y seme 'bastante'. Los sustantivos que generalmente se incorporan refieren a entidades inanimadas [II-105] y a partes del cuerpo [III-17], pero también existen algunos sustantivos con referentes animados que se incorporan (p. ej. ?unak-Pe:p [niño-ver] 'cuidar niños'). El oluteco

\footnotetext{
14 El aplicativo tomo- deriva de la combinación de los aplicativos toj- 'instrumental' y mü:'asociativo'.
} 
es de especial interés desde el punto de vista tipológico porque presentan los cuatro tipos mayores de incorporación propuestos por Mithun (1984) en su tipología de incorporación nominal. Los casos [III-9, III-190] ejemplifican el Tipo I 'formación de terminos nuevos' o 'antipasivo' donde se incorpora un sustantivo genérico y el verbo se intransitiviza. Los casos [II-105, III-17] ejemplifican el Tipo II 'manipulación de caso' o 'ascensión de poseedor' donde se incorpora un sustantivo que deja vacante una posición sintactica que es ocupada por otro argumento. En (8) se ejemplifica el Tipo III 'incorporación para la manipulación de sustantivos en el discurso' donde el

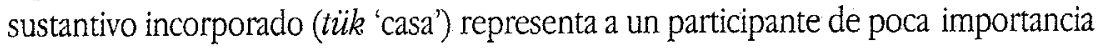
discursiva. Y finalmente en (9) se ejemplifica el Tipo IV 'incorporación con fines clasificatorios' en el cual el sustantivo incorporado (nü: 'agua') refiere a un término genérico que clasifica a una entidad específica que se manifiestan de forma no incorporada ( $k o$ ?ke 'pescado'). Los verbos con incorporación nominal de los tipos II, III, IV no se detransitivizan.

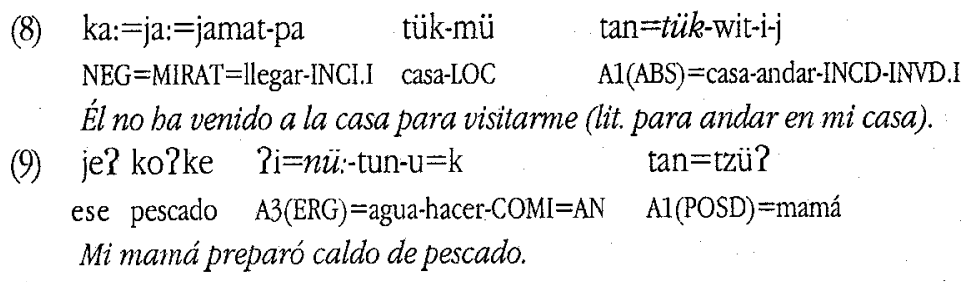

De particular importancia desde el punto de vista tipológico es el hecho de que la lengua incorpora agente (10a-b) además de paciente [II-105], locación o meta (11), y tema de intransitivos (12). La incorporación de agente no se había reportado en lenguas mesoamericanas y los trabajos teóricos sobre incorporación (Mithun 1984, Baker 1988, 1996) ignoran la existencia de este tipo de construcciones que parecen ser bastante raras a nivel tipológico.

(10) a. ta=pu?-jü:n-ü-pa

$\mathrm{B} 1(\mathrm{ABS})=$ arena-tragar-INV-INCI.I

Me está tragando la arena.

b. $\quad$ ta $=$ ?apit-kup-ü-w

$\mathrm{B} 1(\mathrm{ABS})=$ espina-punzar-INV-COMI

Me punzó la espina.

(11) $\tan =$ tewa-mo:y2-am=ak wop-e le:ncho

$\mathrm{A} 3(\mathrm{ERG})=$ nalga $-\mathrm{dar}-\mathrm{IRRI}=\mathrm{AN} \quad$ golpear-NMZR Lencho

Le di de nalgadas a Lencho (lit. Le di de golpes en sus nalgas de Lencbo). 
(12) : ta $=p u ? p u-y e: k-u$

$\mathrm{A1}(\mathrm{ABS})=$ panza-crecer-COMI

Me creció mi panza.

Algunos verbos como tu:t? 'poner' y jik 'untar' que seleccionan agente, tema y locación pueden incorporar tanto el tema como la locación. En (13) los dos participantes incorporados son el tema tzoy 'medicina' y el elemento poseído de la locación $p u ? p u$ 'panza'.

\section{(13) tan=pu?pu-tzoy-tu:t?-u=k le:ncho \\ $\mathrm{A} 1(\mathrm{ERG})=$ panza-medicina-poner-COMI=AN Lencho \\ Le puse medicina a Lencbo en su panza.}

El verbo oluteco incorpora morfemas de partes del cuerpo que especifican la localización de la parte con respecto al todo de manera semejante a otras lenguas mesoamericanas como el totonaco y el tarasco (Levy 1994). Sincrónicamente algunos de estos morfemas están relacionados con sustantivos no incorporados que refieren a partes del cuerpo. Por ejemplo, los prefijos ko?-, win-, yo?k y Paw están relacionados con los sustantivos ko?pak 'cabeza', wintoj 'cara', yo?ktu 'cuello' y Paw 'boca' que pueden aparecer en su forma no incorporada. Dentro de un segundo subgrupo se encuentran morfemas (p. ej. ni: 'cuerpo, superficie' [III-108], ?ü̈x 'espalda', natz 'parte trasera' [III-226]) que no tienen como contraparte un sustantivo homófono que ocurra en su forma no ligada. La semántica de los compuestos morfema_de parte+verbo refleja un grado de lexicalización mayor que la de los otros casos de incorporación nombre+verbo cuyo significado puede aun determinarse a partir del significado de los morfemas que forman el compuesto. Los casos en (14) son ejemplos de bases verbales que incorporan partes:
a. ?aw-pa:t [boca-alcanzar] 'besar'
b. ko?-ju:x [cabeza-cubrir] 'ser madrina de alguien'
c. win-ka?tz [ojo-cortar] 'limpiar la milpa'
d. Püx-poj [espalda-patear] 'patear por detrás'
e. natz-?e:p [atrás-ver] 'espiar'
f. ni:-ta:tz? [cuerpo-orinar] 'orinar a alguien'

\section{ORDEN DE ARGUMENTOS}

El oluteco es una lengua con orden de argumentos flexible. El único argumento central de construcciones monovalentes $(S=$ agente o paciente de intransitivos) 
puede ocurrir antes (SV) [I-53] o después del predicado (VS) [I-3]. En construcciones con verbos bivalentes agentivos (transitivos), bivalentes no agentivos y trivalentes (ditransitivos), los argumentos centrales pueden preceder o seguir al verbo en todos los órdenes lógicamente posibles. En las oraciones con verbos bivalentes no agentivos las distintas posiciones que el tema (T), y la locación o experimentante (L) ocupan con respecto al verbo son las siguientes: TV [1-90], VT [1-87], LV (15), VL (16), VTL (17), VLT (16), TLV (18), LTV (19).

(15) de rrepente tuk 2i=küj-mi:n?-i-j

de repente uno $\mathrm{Al}(\mathrm{ABS})=\mathrm{APL1}$-venir-INCD-INVD.I

... de repente se le viene a uno (una idea).

(16) ta=?it-nü-ü-pa Pü:tz tan=tük de tza:j

$\mathrm{A} 1(\mathrm{ABS})=$ existir-ya-INV-INCl.I yo $\quad \mathrm{A} 1(\mathrm{POSD})=$ casa de piedra

Yo tengo mi casa de piedra.

(17) ?it-ü-w=ak pure:ro-tük tan=?apu

existir-INV-COMI=AN purero-PL $\quad \mathrm{Al}(\mathrm{POSD})=$ abuelo

Mi abuelo two fabricantes de puros.

(18) pu:ro jün-tza:j ya?aj 2it-ü-pa

puro fuego-piedra este existir-INV-INCI.I

Éste tiene puro carbón.

(19) mi:tz jupa? xiwit $\min =$ ?it-i-j

đú cuánto año $\mathrm{A} 2(\mathrm{ABS})=$ existir-COMD-INVD.I

¿Tú cuántos años tienes?

Las oraciones con verbos bivalentes agentivos (transitivos) con dos argumentos presentan los seis órdenes lógicamente posibles. Las distintas posiciones que el agente (A), y el paciente/tema $(\mathrm{O})$ ocupan con respecto al verbo son las siguientes: AOV (20), OAV (21), AVO [I-60], VOA [1-71], VAO [II-59], OVA [II-154], OV [I-131], VO $[\mathrm{I}-1], \mathrm{AV}[\mathrm{I}-21], \mathrm{VA}[\mathrm{I}-50]$.

(20) ja? yaj-tük jaj=ak xokot nükx-pa ta=ka?tz-küx-i

DEF este-PL ese=AN zacate ir-INCI.I $\quad$ C3(ERG) $=$ cortar-3PL-INCD

Los demás van a cortar ese zacate.

(21) tzoy ja?aj ?i-wa:n?-pe

medicina ése A3(ERG)-querer-INCI.T

Ése quiere medicina.

En las oraciones con verbos trivalentes (ditransitivos) algunas de las posiciones alternantes del agente (A), tema/paciente (O2), meta/benefactivo (O1) son las 
siguientes: AO2V (22), AVO2 [III-185], AO1V (23), AV01O2 (24), VO102 [II-105], VO2V1 (25), VO2 [I-166], VO1 [I-6], O2V [II-60], O1VO2 (26), AV [II-68], VA (27).

(22) $\mathrm{mi}: \mathrm{tz}=\mathrm{ak} \quad \min =\mathrm{tzoy} \quad \min =\mathrm{mo}: \mathrm{y} 2-\mathrm{am}$ tú $=\mathrm{AN} \quad \mathrm{A} 2(\mathrm{POSD})=$ medicina $\quad \mathrm{A} 2(\mathrm{ERG})=\mathrm{dar}-\mathrm{IRR}$ Túle vas a dar tu remedio.

(23) ja? mi:tz=ak mi=yak-ke:k?-a?x-ü-pa

éste tú $=$ AN $\quad B 2(A B S)=C A U S-q u i t a r-A P L$-2-INV-INCI.I Éste te lo está quitando a tí.

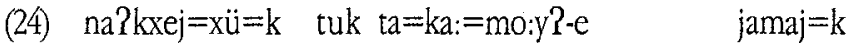
cuando $=\mathrm{EV}=\mathrm{AN} \quad$ uno $\mathrm{C} 3(\mathrm{ERG})=\mathrm{NEG}=\mathrm{dar}=\mathrm{INCD} \quad$ aquel $=\mathrm{AN}$ mu:xi-nak kay-e pájaro-DIM comer-NMZR Cuando uno no le da comida a aquel pajarito.

(25) mo:y?-a ?an+pa-nü: ya?aj=ak na?aw-?unak dar-IMPR ardiente-agua este $=\mathrm{AN} \quad$ viejo-DIM iDále aguardiente a este ancianito!

(26) ya?aj=ak na?aw-?unak $\min =m o: y ?-a m=a k \quad$ ?an+pa-nü: este $=\mathrm{AN} \quad$ viejo-DIM $\quad \mathrm{A} 2(\mathrm{ERG})=\mathrm{dar}-\mathrm{IRR}=\mathrm{AN} \quad$ ardiente-agua A este viejito le vas a dar aguardiente.

(27) ja?mej $\tan =$ nüm-a2x-i-y tan=tükaw asi $\quad \mathrm{A} 1(\mathrm{ABS})=\mathrm{decir}-\mathrm{APL} 2-\mathrm{INCD}-\mathrm{INVD} . \mathrm{C} \quad \mathrm{A} 1(\mathrm{POSD})=$ papá Así me lo dijo mi papá.

El Cuadro 5, que resulta del análisis de los tres textos que presento abajo, resume las ocurrencias de predicados por tipos (monovalentes, bivalentes agentivos y no agentivos, trivalentes) y se dan los totales del número de oraciones con y sin argumentos externos al predicado. El gran número de oraciones que expresan los argumentos únicamente en el verbo muestran el carácter de una lengua con marcación en el núcleo (Nichols 1986, DuBois 1985). Del total de 591 oraciones analizadas, 253 (43\%) no expresan ninguno de los argumentos verbales por medio de sustantivos, pronombres u oraciones de complemento. Dentro del mismo corpus 130 oraciones (22\%) [ 7 bivalentes no agentivas, 96 bivalentes agentivas, 27 trivalentes] no expresan uno o dos de los argumentos seleccionados por el verbo por medio de sustantivos, pronombres u oraciones de complemento. Finalmente, 208 (35\%) del total de las oraciones [185 intransitivas, 1 bivalente no agentiva, 22 bivalentes agentivas] expresan de manera externa todos los argumentos seleccionados por el verbo. 


\begin{tabular}{|c|c|c|c|c|c|c|}
\hline & Monovalente & $\begin{array}{l}\text { Bivalente } \\
\text { no-agentivo }\end{array}$ & $\begin{array}{l}\text { Bivalente } \\
\text { agentivo }\end{array}$ & Trivalente & Total & \\
\hline 0 arg. & 185 & 2 & 61 & 5 & 253 & $43 \%$ \\
\hline 1 arg. & 185 & $\begin{array}{l}7(\mathrm{~T}: 7) \\
(\mathrm{L}: 0)\end{array}$ & $\begin{array}{r}96 \text { (A: 23) } \\
(\mathrm{O}: 73)\end{array}$ & $\begin{array}{r}24(\mathrm{~A}: 4) \\
(\mathrm{O} 2: 16) \\
(01: 4)\end{array}$ & 312 & $53 \%$ \\
\hline 2 arg. & - & 1 & 22 & $\begin{array}{r}3(\mathrm{~A}: 1) \\
(\mathrm{O} 2: 3) \\
(\mathrm{O} 3: 2)\end{array}$ & 26 & $4 \%$ \\
\hline 3 arg. & - & - & - & 0 & 0 & $0 \%$ \\
\hline Total & 370 & 10 & 179 & 32 & 591 & $100 \%$ \\
\hline
\end{tabular}

El Cuadro 6, basado en el mismo corpus que el Cuadro 5, resume la distribución de los argumentos que se manifiestan por medio de pronombres, sustantivos u oraciones de complemento.

\begin{tabular}{lllll}
\multicolumn{5}{c}{ CUADRO 6. ORDEN DE ARGUMENTOS S/A/O/T/L/OI/O2. } \\
& Monovalente & $\begin{array}{l}\text { Bivalente } \\
\text { no-agentivo }\end{array}$ & $\begin{array}{l}\text { Bivalente } \\
\text { agentivo }\end{array}$ & Trivalente \\
& & - & 21 & 3 \\
$(\mathrm{~S} / \mathrm{A}) \mathrm{V}$ & 32 & - & 24 & 2 \\
$\mathrm{~V}(\mathrm{~S} / \mathrm{A})$ & 153 & $3(\mathrm{~T}: 3)$ & 19 & $3(02: 2)$ \\
$(\mathrm{O} / \mathrm{T} / \mathrm{L} / \mathrm{O} / \mathrm{O} 2) \mathrm{V}$ & - & $(\mathrm{L}: 0)$ & & $(01: 1)$ \\
& & $6(\mathrm{~T}: 5)$ & 76 & $22(02: 17)$ \\
$\mathrm{V}(\mathrm{O} / \mathrm{T} / \mathrm{L} / \mathrm{O} 1 / \mathrm{O} 2)$ & - & $(\mathrm{L}: 1)$ & & $(01: 5)$
\end{tabular}

Dentro de los tres textos analizados si se considera únicamente el grupo de oraciones transitivas (que incluyen verbos bivalentes agentivos y trivalentes), como lo sugiere la tipología tradicional que presupone seis órdenes básicos, se puede observar que sólo un $11 \%$ de estas oraciones (23 del total de 211 oraciones transitivas) expresan los argumentos $\mathrm{A}$ y $\mathrm{O}(\mathrm{O} 1 \mathrm{y} / \mathrm{o} \mathrm{O} 2$ en ditransitivos) externos al predicado. Dentro de este subgrupo hay una clara preferencia por el patrón AVO (59\%). Sin embargo, cuando se agrupan por un lado (como lo sugiere Dryer 1997) los argumentos $S$ de verbos intransitivos con los argumentos $A$ de predicados bivalentes y trivalentes, mientras que por otra parte se agrupan los argumentos $O$ de verbos transitivos con los argumentos $01, \mathrm{O} 2$ de trivalentes y los argumentos T y $\mathrm{L}$ de predicados bivalentes no agentivos, se puede observar que la lengua tiene una clara 
preferencia por los patrones VS y VO (Cuadro 7). Esto quiere decir que el oluteco es una lengua con orden de argumentos flexible con fuerte tendencia a colocar al verbo en posición inicial. Habría que apuntar, sin embargo, que el patrón VS/A (76\%) es más común que el patrón S/AV (24 \%) en el Cuadro 7 debido a la tendencia ascendrada de los argumentos $S$ de intransitivos $(153=82 \%$ del total de $S$ intransitivos) a ocupar la posición posverbal. Las frecuencias muestran que entre los argumentos $\mathrm{A}$ de las oraciones transitivas no hay ninguna clara preferencia hacia la posición preverbal $(24=46 \%)$ o posverbal $(26=54 \%)$.

CUADRO 7. FRECUENCAASDE ÓRDENES DEARGUMENTOSS/A/O.

$\begin{array}{rrrrrrr}\text { AVO } & 13 & (59 \%) & \text { S/AV } & 56(24 \%) & \text { OV } & 24(19 \%) \\ \text { VOA } & 5 & (23 \%) & \text { VS/A } & 178(76 \%) & \text { VO } & 103(81 \%) \\ \text { VAO } & 2 & (10 \%) & & & & \\ \text { AOV } & 1 & (5.5 \%) & & & & \\ \text { OVA } & 1 & (5.5 \%) & & & & \end{array}$

El Cuadro 7 muestra claramente que el orden VO es estadísticamente cuatro veces más frecuente que el orden OV. El patrón VO, que es el preferido sincrónicamente, está en conflicto con varios de los rasgos estructurales de la lengua (Cf. Comrie 1981:80-97, Campbell, L et al. 1986: 547.548, Dryer 1997). Los rasgos estructurales del oluteco que son prototípicos de lenguas del tipo OV son los siguientes: a) posposiciones [I-51, I-193, II-77, II-3, III-156], b) el poseedor precede al sustantivo poseído en sintagmas [II-107] y en compuestos nominales [III-60], c) el poseedor precede a los sustantivos relacionales [I-171, II-78, III-14], d) el adjetivo precede al sustantivo [II-103, III-145], e) el modificador calificativo precede al sustantivo en compuestos nominales [II-31], f) el verbo principal precede a los auxiliares flexionados [I-19, Il-109], g) el sustantivo incorporado precede a la raíz verbal [II-105, III-9, III-17, III-105], y h) los adverbios de manera preceden al verbo [II82, III-192, III-240]. Aparte de la fuerte tendencia a colocar el argumento $O$ en posición posverbal ( $81 \%$ del total), el oluteco tiene una serie de rasgos estructurales típicos de las lenguas del tipo VO: a) nuevas preposiciones nativas y prestadas del español [II85, III-1, III-89, III-117], b) orden innovador en sintagmas de posesión donde el sustantivo poseído precede al sustantivo poseedor [III-61, III-78, III-108], c) el núcleo del sintagma nominal precede a la oración de relativo [I-184, III-28, III-231], d) los auxiliares léxicos preceden al verbo principal [I-46, I-113, III-35, III-135], e) el adverbio de manera le sigue al verbo [III-87, III-109, III-191], f) las oraciones de propósito le siguen al verbo matriz de movimiento [III-33, III-34], y g) las oraciones de complemento le siguen al verbo matriz [II-47, III-57/58, III68/69, III-71/72]. Todas 
estas características estructurales son evidencia de que el oluteco o quizá su predecesor, el proto-mixe-zoque, fue una lengua (S/A)OV que adquirió los rasgos VO debido al contacto con lenguas del área mesoamericana que son prototípicamente lenguas con verbo inicial (Campbell et al 1986).

\section{DISCONTINUIDAD DE CONSTTTUYENTES}

Una última característica de la estructura gramatical del oluteco que merece ser mencionada, debido a que no se ha discutido en otras lenguas mesoamericanas, es el hecho de que el conjunto de palabras que forman unidades semánticas y que en la mayor parte de las lenguas se manifiestan como constituyentes sintácticos (i.e. sintagmas nominales, verbales, etc.) pueden aparecer en oraciones de manera discontinua: Los siguientes son casos donde un elemento de la expresión nominal precede al predicado mientras que el otro elemento, con el que semánticamente forma una unidad, le sigue: determinante y sustantivo [I-28, III-24, (28)], sustantivo coordinado con otro sustantivo [I-180], mensurativo y sustantivo [II-62], cuantificador y sustantivo (29)-(31), numeral y sustantivo (32)-(34), sustantivo poseedor y sustantivo poseído (35), sustantivo relacional y sustantivo poseedor (36)(38), adposición y sustantivo (39)-(40). Las palabras en letras cursivas en los ejemplos (28) a (40) representan los elementos de las expresiones nominales discontinuas.

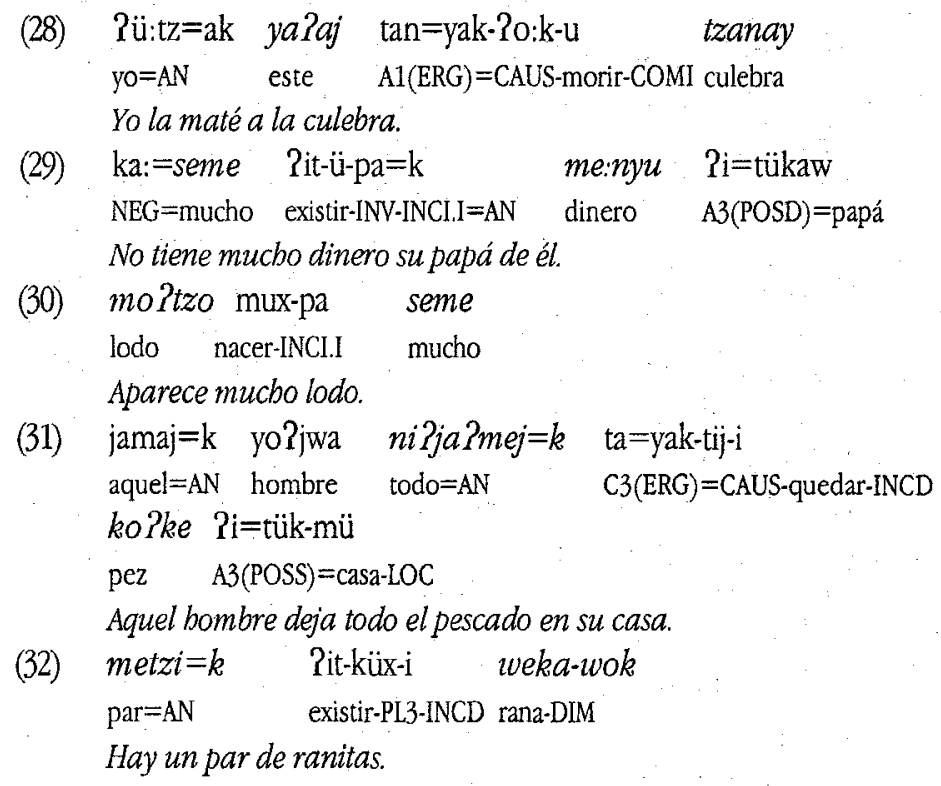


ENTRE CONEJOS, DLABLOS Y VENDEDORES DE CACA

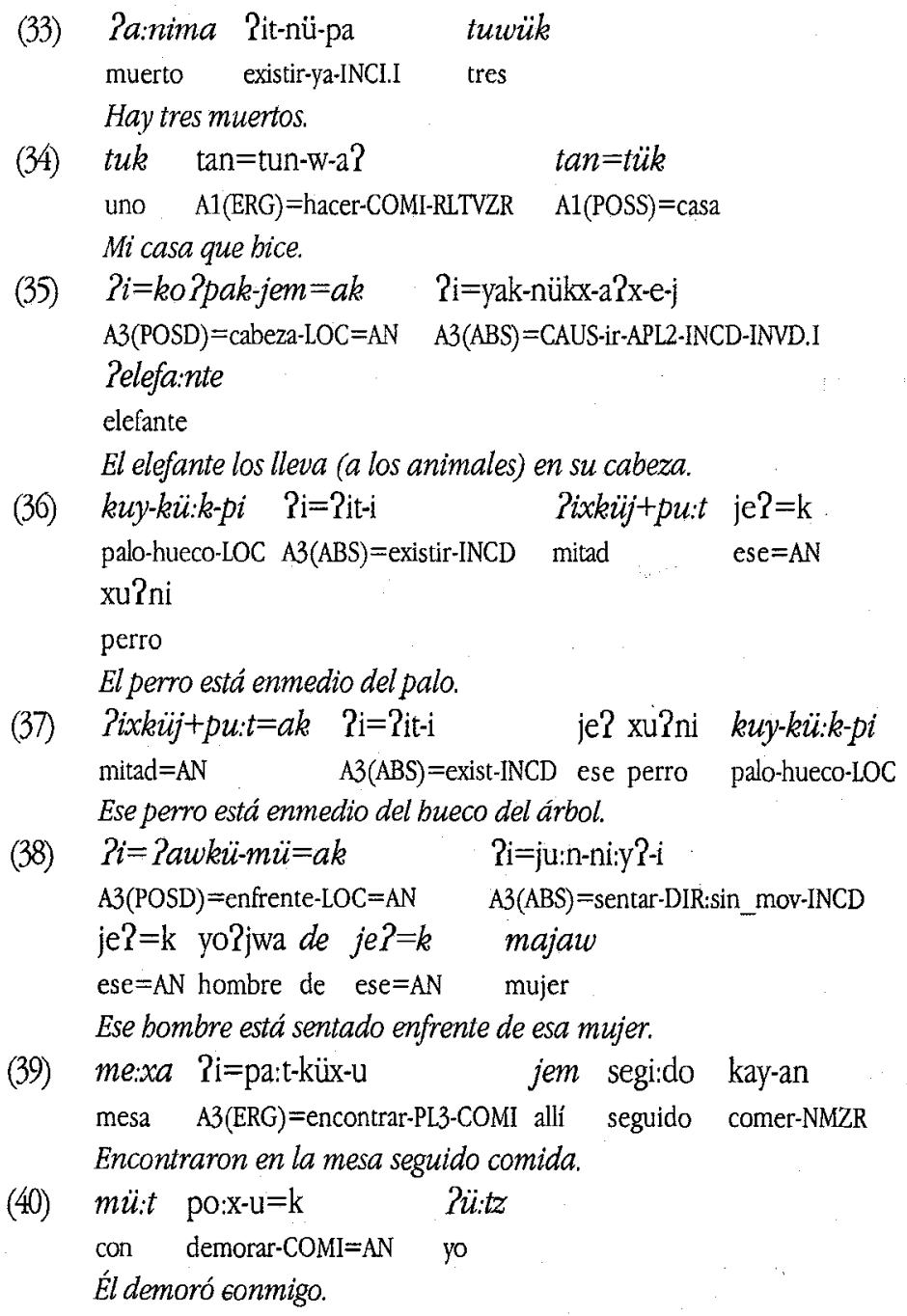

Estos ejemplos que provienen de conversaciones y narraciones hacen patente dos regularidades con respecto a las expresiones discontinuas en oluteco. Primero, los elementos discontinuos ocurren únicamente con los siguientes roles gramaticales: paciente de transitivos, único argumento de intransitivos y al tema de ditransitivos y bivalentes no agentivos. Es decir, no hay discontinuidad de elementos nominales que refieran al agente o al benefactivo. Segundo, todas las ocurrencias de elementos discontinuos siguen un patrón $i$-V-i. Es decir, no hay discontinuidad 
cuando los dos elementos correferenciales preceden, $i-\mathrm{X}-i \mathrm{-V}$, o siguen, $\mathrm{V}-i-\mathrm{X}-i$, al verbo. ${ }^{15}$

\section{LOS TRES CUENTOS}

\section{EL CONEJO QUE QUERÍA CRECER}

1. tankapxam ya?aj, tu?k kwe:ntona?k

$$
\text { tan=kapx-am ya?aj tuk kwe:nto-nak }
$$

$A 1(E R G)=h a b l a r-I R R I$ este uno cuento-DIM

1. Voy a decir este cuentito.

2. jaj ya?aj kwe:nto 3. nümpaxü?k koya

jaj ya?aj kwe:nto nüm-pa $=\mathrm{xü}=\mathrm{k}$ koya

ese este cuento decir-INCI.I=EV=AN conejo

2. Este es el cuento. 3. Dice el conejo:

4. tachu:chuchikna? seme 5. nümpaxü?k koya ta $=$ chu:chu-chik-na? seme nüm-pa $=x \ddot{u}=k$ koya

$\mathrm{B} 1(\mathrm{ABS})=$ chico-DIM-ADVZR muy decir-INCI.I $=\mathrm{EV}=\mathrm{AN}$ conejo

4. -Soy muy cbiquitito-, 5. dizque dice el conejo.

6. pero tükxa:mak xnüme? je?k je:fe pero ta $=$ nükx-am $=$ ak tax $=$ nüm-a?-eje $?=\mathrm{k}$ jefe

pero $\quad B 1(A B S)=i r-I R R I=A N \quad C 1(E R G)=$ decir-APL2-INCD ese $=$ AN jefe 6. -Pero voy a decirle a ese jefe (a Dios),

7. ?e:pena 8. ta tayakye:küpak ma:s

2e:p-pe=na ta ta=yak-ye:k-ü-pa $=k \quad$ ma:s

ver -INCI.T=todavía COND B1(ABS)=CAUS-crecer-INV-INCI.I=AN más

7. vamos a ver (a ver que dice), 8. si me bace más grande-,

9. nümpaxü ja? 10. je? ?u:raxükü ?ipeti nüm-pa $=x \ddot{u} \quad$ ja? je? ?u:ra $=x \ddot{u}=\mathrm{k} \quad$ ? $\mathrm{i}=$ pet- $\mathrm{i}$

decir-INCL. $=\mathrm{EV}$ él ese hora $=\mathrm{EV}=\mathrm{AN} \quad \mathrm{A} 3(\mathrm{ABS})=$ subir-COMD

9. dizque dice él. 10. A esa hora dizque se subió.

15 Uso la vocal $i$ en cursivas para referirme al elemento correferencial dentro de las expresiones nominales. El símbolo X refiere a cualquier otro constituyente de la oración que hipotéticamente pudiera intervenir entre los elementos correferenciales de las expresiones nominales. 

11. ?ijamatixui?k yukpi
?i $=$ jamat $-\mathrm{i}=\mathrm{xu}=\mathrm{k}$
yuk-pi
11. Dizque llegó arriba. 12. Dizque dice:
13. tawampe
14. ti?k ta?nü?k
tan=wa:n?-pe
ti?k ta?nük
$\mathrm{Al}(\mathrm{ERG})=$ querer-INCI.T
CFCT grande
13. -Quiero 14. ser grande.
15. taxyakye:ka?ne?k
tax=yak-ye:k-a?n-ek
$\mathrm{C} 1$ (LOCAL) $=$ CAUS-crecer-IRRD-INV.LOCAL
15. -Quiero que tú me bagas crecer

12. nümpaxükü

nüm-pa $=\mathrm{xü}=\mathrm{k}$

$\mathrm{A} 3(\mathrm{ABS})=$ llegar- $\mathrm{COMD}=\mathrm{EV}=\mathrm{AN}$ arriba- $\mathrm{LOC} \cdot$ decir $-\mathrm{INCI} \mathrm{I}=\mathrm{EV}=\mathrm{AN}$

16. porke tachu:chu seme $\quad$ 17. jama:k ma:s ta?jitikajü porke ta $=$ chu:chu seme jamaj $=\mathrm{k}$ ma:s ta?jitik-2aj porque $\mathrm{B} 1(\mathrm{ABS})=$ chico muy aquel=AN más grandes-RLTVZR

16. porque soy muy chiquito. 17. Los más grandes

18. tamü:piyü?küpa?

$\mathrm{ta}=$ mü:-piyü?k-ü-pa $=\mathrm{a}$ ?

$\mathrm{B} 1(\mathrm{ABS})=\mathrm{APL} 3$-correr-INV-INCL. $\mathrm{I}=\mathrm{AN}$

18. me corretean.

19. takoxi?o:küpa?

$\mathrm{ta}=\mathrm{kox}-\mathrm{i}-\mathrm{Po}: \mathrm{k}-\mathrm{u}-\mathrm{pa}=\mathrm{a}$ ?

$\mathrm{B1}(\mathrm{ABS})=$ golpear_con_puño-NMZR-DESID-INV-INCI.I=AN

19. Me quieren golpear

20. porke chu:chu ta?e:pej

porke chu:chu $\tan =$ ?e:p-e-j

porque chico $\quad \mathrm{Al}(\mathrm{ABS})=$ ver-INCD-INVD.I

20. porque me ven chiquito.

21. je?k ta?jitikajü tayakpiyü?küxüpa?

je?=k ta?jitik-2aj ta=yak-piyü?k-küx-ü-pa $=a ?$

ese $=\mathrm{AN}$ grandes-RLTVZR $\mathrm{B1}(\mathrm{ABS})=$ CAUS-correr-PL3-INV-INCI.I $=\mathrm{AN}$

21. Los más grandes me corretean.

22. tej pe? taxakye:kama?k ya?jü

te?ej pe? tax=yak-ye:k-am-ak ya?aj

ahora de verdad C1(LOCAL) $=$ CAUS-crecer-IRRI-INV.LOCAL éste

22. ¿Y abora, qué no me vas a bacer crecer? 

23. porke tachu:chu seme …24. tanüme?xü?k je:fe porke $t a=c h u: c h u$ seme $t a=n u ̈ m-a ?-i=x \ddot{u}=k \quad$ je:fe porque $\mathrm{B} 1(\mathrm{ABS})=$ chico muy $\quad \mathrm{C} 3(\mathrm{ERG})=$ decir $-\mathrm{APL} 2-\mathrm{INCD}=\mathrm{EV}=\mathrm{AN}$ jefe 23. Porque soy muy cbico-, 24. dizque le dice al jefe (a Dios).

\begin{tabular}{|c|}
\hline 25. nümpaxü?k je:fe \\
\hline nüm-pa $=x \ddot{u}=k$ \\
\hline decir-INCI.I $=\mathrm{EV}=\mathrm{AN}$ \\
\hline
\end{tabular}

25. Dizque dice el jefe (Dios): 26. -Está bien, 27. está bien.

28. pero nomaskoj ja?k tawampe ka:ja?w mü:tak tu:ru mü:tak ?uxpi?n pero nomas $=\mathrm{koj}$ ja? =k $\tan =$ wa:n?-pe ka:jaw pero nomás-nomás $\mathrm{DEF}=\mathrm{AN} \mathrm{A} 1(\mathrm{ERG})=$ querer-INCI.T tigre mü:t=ak tu:ru mü:t=ak Puxpin $\mathrm{y}=\mathrm{AN}$ toro $\mathrm{y}=\mathrm{AN}$ lagarto

28. Pero solamente quiero nomás (que me traigas) tigre, toro y cocodrilo.

29. Pal mixmü:mi?nanak je?ej

Pal mix=mü:-mi:n?-a?n=ak je?ej

a) $\mathrm{C} 2(\mathrm{ERG})=\mathrm{APL} 3$-venir-IRRD $=$ ANese

29. En cuanto traigas eso,

30. Pento:nses taxyakye:kam

Pento:nses tax=yak-ye:k-am

entonces $\quad \mathrm{C} 1(\mathrm{LOCAL})=\mathrm{CAUS}$-crecer-IRRI

30. entonces te voy a bacer crecer.

31. ta?nü?k mijata?n

ta?nük min=jat-a?n

grande $\mathrm{A} 2(\mathrm{ABS})=$ volverse-IRRD

31. Vas a ser grande-

32. je? ?u:raxü?k ?ika?i je?k koya

je? $2 \mathrm{u}: \mathrm{ra}=\mathrm{xü}=\mathrm{k} \quad \mathrm{i}=\mathrm{ka} ? \mathrm{i} \quad \mathrm{je} \quad \mathrm{e}=\mathrm{k}$ koya

ese hora $=\mathrm{EV}=\mathrm{AN} \quad \mathrm{A} 3(\mathrm{ABS})=$ bajar-COMD ese $=\mathrm{AN}$ conejo

32. A esa bora dizque bajó (del cielo) ese conejo

33. mü:t je? ?u:rak Piyaknüma?xiy

mü:t je? ?u:ra=k ?i=yak-nüm-a?x-i-y

y ese hora=AN A3(ABS)=PASV-decir-APL2-COMD-INVD.C

33. y a esa bora se le dijo 
34. ?imi?nanak tapüki je?k ka:ja?w mü:tak tu:ru mü:tak ?uxpi?n

$$
\begin{aligned}
& \text { ?i=mi:n?-a?n=ak ta=pük-i je?=k ka:jaw } \\
& \mathrm{A} 3(\mathrm{ABS})=\text { venir-IRRD }=\mathrm{AN} \quad \mathrm{C3}(\mathrm{ERG})=\text { agarrar }-\mathrm{INCD} \text { ese }=\mathrm{AN} \text { tigre } \\
& \text { mü:t=ak tu:ru mü:t=ak Puxpin } \\
& \mathrm{y}=\mathrm{AN} \text { toro } \mathrm{y}=\mathrm{AN} \text { lagarto } \\
& \text { 34. que viniera a traer a ese tigre y a el toro y a el cocodrilo. }
\end{aligned}
$$

35. mü:t ?al ?ika?ikü de wep yukpi

$$
\begin{aligned}
& \text { mü:t ?al } \quad \text { i }=k a ?-i=k \quad \text { de wew-pi yuk-pi } \\
& \text { y al } A 3(A B S)=\text { bajar-COMD }=A N \text { de allá-LOC arriba-LOC } \\
& \text { 35. Y en cuanto bajó de allá arriba, }
\end{aligned}
$$

36. 2al 2ika?ne:kü

Pal $2 \mathrm{i}=\mathrm{ka} 2-\mathrm{nü}-\mathrm{i}=\mathrm{k}$

al $\mathrm{A} 3(\mathrm{ABS})=$ bajar-ya $-\mathrm{COMD}=\mathrm{AN}$

36. en cuanto bajó,

37. ?iwitixükoj pi:na?k

$$
\mathrm{Pi}=\text { wit-i=xü=koj pi:nak }
$$

$\mathrm{A} 3(\mathrm{ABS})=$ andar $-\mathrm{COMD}=\mathrm{EV}=$ nomás poco

37. dizque que en cuanto caminó un poquito nomás,

38. tapa:tixük ja?xükü ka:ja?w

$$
\mathrm{ta}=\mathrm{pa}: \mathrm{t}-\mathrm{i}=\mathrm{xu}=\mathrm{k} \quad \mathrm{ja} \mathrm{a}=\mathrm{xu}=\mathrm{k} \quad \text { ka:jaw }
$$

$\mathrm{C} 3(\mathrm{ERG})=$ encontrar $-\mathrm{COMD}=\mathrm{EV}=\mathrm{AN} \quad \mathrm{DEF}=\mathrm{EV}=\mathrm{AN}$ tigre

38. dizque encontró a el tigre.

39. semexükokü komo jüyta?ki?o:kpaxü ja? seme $=x \ddot{u}=k o j=k$ komo jüy+ta:k?-i-Po:k-pa=xü ja? muy $=\mathrm{EV}=$ nomás $=\mathrm{AN}$ como jugar-NMZR-DESID-INCLII=EV él 39. Dizque (el tigre) nomás como que quiere jugar mucho.

40. jemxü?k ?ijanechipti? $j e m=x \ddot{u}=k \quad$ ?i $=$ jane-chip-ti:y? $-\mathrm{i}$

alli $=\mathrm{EV}=\mathrm{AN} \quad \mathrm{A} 3(\mathrm{ABS})=$ simulando-arunar-PUNTL-INCD

40. Abi anda aruñando de a mentiritas.

41. ?ijanewopti? je?k ka:ja?w

$$
\begin{aligned}
& 2 \mathrm{i}=\mathrm{jane}-\text {-wop-ti:y?-i je } \mathrm{i}=\mathrm{k} \quad \text { ka:jaw } \\
& \mathrm{A} 3(\mathrm{ABS})=\text { simulando-golpear-PUNTL-INCD ese }=\mathrm{AN} \text { tigre } \\
& \text { 41. Ese tigre andaba rascando de a mentiritas }
\end{aligned}
$$


42. ta?e:pi? koyana?k

ta=?e:p-?i:y?-i koya-nak

$\mathrm{C} 3(\mathrm{ERG})=$ ver-DIR:adentro-COMDconejo-DIM

42. cuando el conejito lo vió.

43. nümpaxü?k koya

nüm-pa $=\mathrm{xü}=\mathrm{k}$ koya

decir-INCII $\mathrm{I}=\mathrm{EV}=\mathrm{AN}$ conejo

43. Dizque dice el conejo:

44. ka?jüyta?kü 45. taka?jüyta?ki?o:kpa

$k a:=j u ̈ y+t a: k ?-\ddot{u} \quad t a=k a:=j u ̈ y+t a: k 2-i-2 o: k-p a$

NEG $=$ jugar-IMPR $\quad B 1(\mathrm{ABS})=\mathrm{NEG}=$ jugar-NMZR-DESID-INCI.I

44. -iNo estés jugueteando! 45. No quiero jugar.

46. minukoje? xnüme?

mi:n?-u=koj=je? tax=nüm-a?-e

venir-COMI=nomás=ese $\mathrm{C} 1(\mathrm{LOCAL})=$ decir-APL2-INCD

46. Vine nomás a decirte eso:

47. japomü tiwi?k ?inaxa?n ja:mu

japo:m tüw-ik $\quad$ i $=$ nax-a?n ja:mu

mañana apretar-PCP $\mathrm{A} 3(\mathrm{ABS})=$ pasar-IRRD viento

47. "Mañana, va a pasar fuerte el viento.

48. tiwi?k ?inaxa?n ja:mu japom

tüw-ik $\quad$ i $=$ nax-a?n ja:mu japo:m

apretar-PCP $\mathrm{A} 3(\mathrm{ABS})=$ pasar-IRRD viento mañana

48. Va a pasar el viento fuerte mañana,

49. mü:t minükxam

mü:t mi=nükx-am

y $\quad$ B2(ABS) $=\mathrm{ir}-\mathrm{IRRI}$

49. $y$ te vas a ir (con el viento).

50. mimü:nükxa:nüp ya?k ja:mu

mi=mü:-nükx-an-ü-pa $\quad$ ya2aj=ak ja:mu

$B 2(A B S)=A P L 3-I r-I R R-I N V-I N C I I$ éste $=A N \quad$ viento

50. Te va a llevar el viento

51. wep minükxa?n ko:taspetpa? kuyjem

wew-pi min=nükx-a?n ko:+tatz-pet-pa-? kuy-jem

allá-LOC A2(ABS)=ir-IRRD golpearse-DIR:arriba-INCII.NMZR palo-LOC

51. Allá te vas a ir golpeándote en el palo, 
52. mü: jem miro:ka?n

mü:t jem $\min =20: k-a ? n$

y allí $\mathrm{A} 2(\mathrm{ABS})=$ morir-lRRD

52. $y$ alli te vas a morir

53. porke jamaj ja:mu tiwi?k Pinaxa?n

porke jamaj ja:mu tüw-ik $\quad 2 \mathrm{i}=$ nax-a?n

porque aquel viento apretar-PCP $\mathrm{A} 3(\mathrm{ABS})=$ pasar-IRRD

53. porque ese viento va a pasar fuerte."

54. nümpaxü koya $\quad 55$. so:lo taxtzuma?n

nüm-pa=xü koya so:lo tax=tzum-a?n

decir-INCI.I=EV conejo sólo $\mathrm{C1}(\mathrm{LOCAL})=$ amarrar-IRRD

54. Dizque dice el conejo: 55. -Sólamente que te amarre yo,

56. ja?mej mika?mü:nükxüp ja:mu

ja?mej mi=ka:=mü:-nükx-ü-pa ja:mu

así $\quad \mathrm{B} 2(\mathrm{ABS})=\mathrm{NEG}=\mathrm{APL} 3$-ir-INV-INCLI $I$ viento

56. de esa manera no te lleva el viento-.

57. nümpaxükü je?k ka:ja?w

nüm-pa $=\mathrm{xü}=\mathrm{k} \quad$ je? $=\mathrm{k} \quad$ ka:jaw

decir- $-\mathrm{NCCI} \mathrm{I}=\mathrm{EV}=\mathrm{AN}$ ese $=\mathrm{AN}$ tigre

57. Dizque dice el tigre:

58. jo:ne tzumü?kna 59. nümpaxü ja?

jo:ne tzum-ü-k=na nüm-pa $=x \ddot{u}$ ja?

pues amarrar-IMPR-INV.LOCAL=todavía decir-INCI.I=EV él

58. -iPues amárrame pues!-, 59. dizque dice él.

60. mü:tak je? koya Pipüki?atpe tüpxi

mü:t=ak je? koya $\mathrm{i}=$ =ük-i-?at-pe tüpx-i

$\mathrm{y}=\mathrm{AN}$ ese conejo $\mathrm{A} 3(\mathrm{ERG})=$ agarrar-NMZR-considerar-INCLIT torcer-NMZR

60. Yese conejo traía reata.

61. nümpaxükü koya 62. taxtzuma:m

nüm-pa $=x \ddot{u}=\mathrm{k}$ koya tax=tzum-am

decir-INCI. $\mathrm{I}=\mathrm{EV}=\mathrm{AN}$ conejo $\mathrm{Cl}(\mathrm{LOCAL})=$ amarrar-IRRI

61. Dizque dice el conejo: 62. - Te voy a amarrar-.

63. je? ?u:raxü?k tatzumi

je? ?u:ra $=x \ddot{u}=k \quad t a=t z u m-i$

ese hora $=\mathrm{EV}=\mathrm{AN} \quad \mathrm{C} 3(\mathrm{ERG})=$ amarrar $-\mathrm{COMD}$

63. Dizque a esa bora lo amarró. 


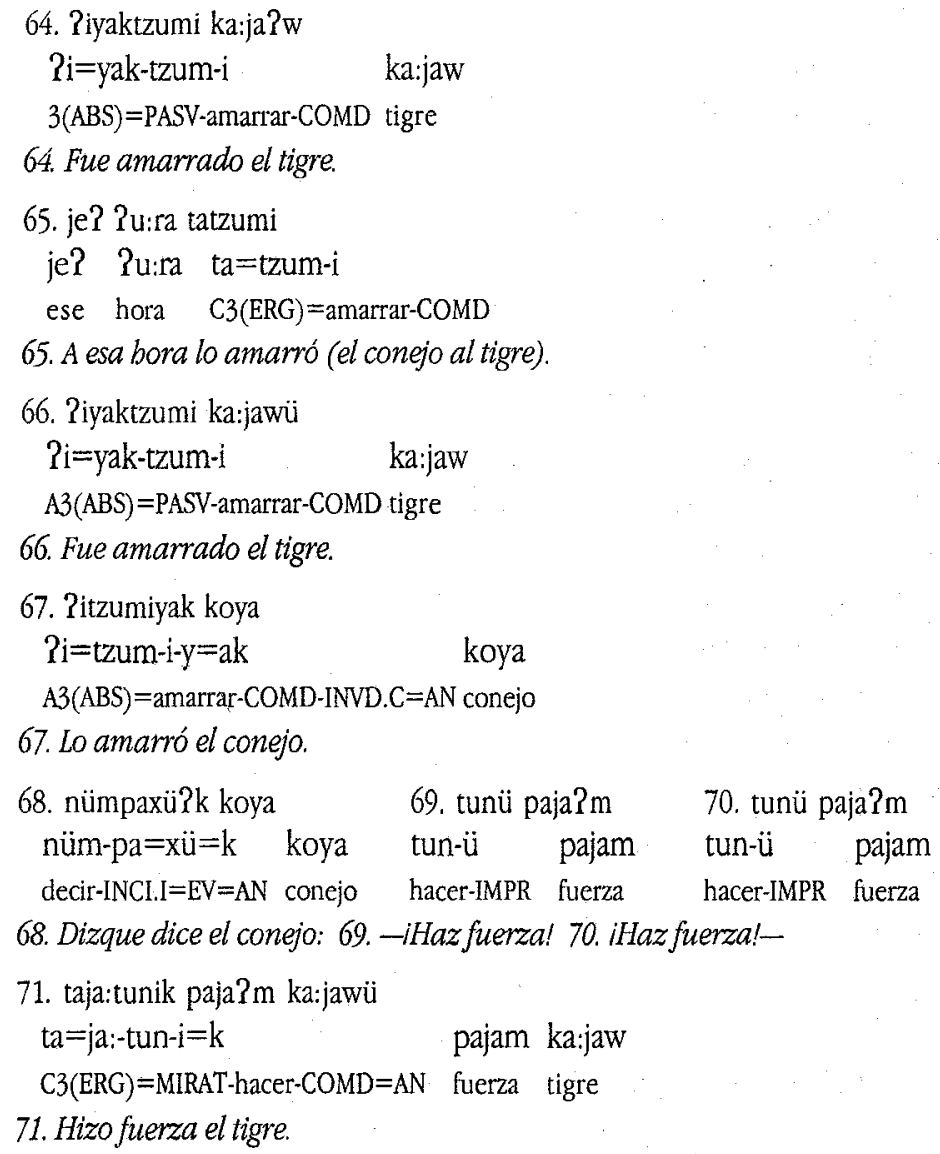


78. tunü ta?na 79. Re:pena

tun-ü ta?na?e:p-pe=na

hacer-IMPR muchover-INCI.T=todavía

78. -iHaz mucha (fuerza)! 79. Vamos a ver

80. ta miyakpotpe ya?aj tüpxi

$\begin{array}{ll}\text { ta } \min =y a k-p o t-p e \quad \text { ya aj } & \text { tüpx-i } \\ \text { COND A2(ERG)=CAUS-reventar-INCI.T este } & \text { torcer-NMZR }\end{array}$

80. si revientas esta reata,-

81. nümpaxük koya

nüm-pa $=\mathrm{xü}=\mathrm{k}$ koya

decir-INCLII $=\mathrm{EV}=\mathrm{AN}$ conejo

81. dizque dice el conejo.

82. taja:tunixü?k paja?m jama:k ka:ja?w

$\mathrm{ta}=\mathrm{ja}$-tun- $\mathrm{i}=\mathrm{xu}=\mathrm{k} \quad$ pajam jamaj=k ka:jaw

$\mathrm{C} 3(\mathrm{ERG})=\mathrm{MIRAT}$-hacer $-\mathrm{NNCD}=\mathrm{EV}=\mathrm{AN}$ fuerza aquel=AN tigre

82. Dizque bacía fuerza aquel tigre.

83. 2ika?yakpotuk tüpxi

$\mathrm{i} \mathrm{i}=\mathrm{ka}:=$ yak-pot- $\mathrm{u}=\mathrm{k} \quad$ tüpx-i

$\mathrm{A} 3(\mathrm{ERG})=\mathrm{NEG}=\mathrm{CAUS}-$ reventar-COMI$=\mathrm{AN}$ torcer-NMZR

83. No reventó la reata.

84. je? ?u:rak koya tyakpitzümi ?ikuchi:nu

je? ?u:ra=k koya ta=yak-pitzüm-i $\quad$ i=kuchi:nu

ese hora $=\mathrm{AN}$ conejo $\mathrm{C} 3(\mathrm{ERG})=\mathrm{CAUS}$-salir-COMD $\mathrm{A} 3(\mathrm{POSD})=$ cuchillo

84. A esa bora el conejo sacó su cucbillo,

85. tani:yo:ki ja?

$\mathrm{ta}=\mathrm{ni}:-\mathrm{yo}: \mathrm{k}-\mathrm{i} \quad \mathrm{ja}$ ?

C3(ERG) =SUPERF-descuartizar-COMD él

85. y lo peló (le quitó la piel al tigre).

86. jemxük ?itiji ka:ja?w sin ?akü

jem $=\mathrm{xü}=\mathrm{k}$ ?i=tij-i ka:jaw sin $2 \mathrm{ak}$

alli $=\mathrm{EV}=\mathrm{AN} \quad \mathrm{A} 3(\mathrm{ABS})=$ quedar $-\mathrm{COMD}$ tigre sin piel

86. Abi dizque quedó el tigre sin cuero.

87. ka:ja?itüpak Pi?akü

$\mathrm{ka}:=\mathrm{ja}:=$ ?it-ü-pa $=\mathrm{k} \quad \mathrm{i} \quad$ = $\mathrm{ak}$

NEG $=$ MIRAT $=$ existir-INV-INCI I $=A N \quad A 3(P O S D)=$ piel

87. Ya no tiene su cuero (el tigre). 
88. je? ?u:ra nümpaxü?k koya

je? ?u:ra nüm-pa $=x u ̈=k k o y a$

ese hora decir-INCIII=EV=AN conejo

88. A esa bora dizque dice el conejo:

89. tajo:yüpak ja:mesko

$\mathrm{ta}=$ jo:y-ü-pa $=\mathrm{k} \quad$ ja:-metzko

$\mathrm{B} 1(\mathrm{ABS})=$ faltar-INV-INCI.I$=\mathrm{AN}$ otro-dos

89. Me faltan otros dos-.

90. nümpaxü ja? 91. ja:tu:ru tajo:yüp

nüm-pa=xü ja? jaj tu:ru ta=jo:y-ü-pa

decir-INCIII=EV él ese toro B1(ABS)=faltar-INV-INCI.I

90. Dizque dice él: 91 . -Me falta el toro-

92. Pinükxixü?k ?iwiti je?k koya

$$
\mathrm{i} i=\text { nükx } \mathrm{i}=\mathrm{x} \ddot{\mathrm{u}}=\mathrm{k} \quad \mathrm{i}=\text { wit-i } \quad \mathrm{je}=\mathrm{k} \text { koya }
$$

$\mathrm{A} 3(\mathrm{ABS})=\mathrm{ir}-\mathrm{INCD}=\mathrm{EV}=\mathrm{AN} \quad \mathrm{A} 3(\mathrm{ABS})=$ andar $-\mathrm{INCD}$ ese $=\mathrm{AN} \quad$ conejo

92. Dizque iba caminando el conejo

93. jemxï?k tapa:ti tu:ru

jem $=x \ddot{u}=k \quad t a=p a: t-i$

tu:ru

alli $=\mathrm{EV}=\mathrm{AN} \quad \mathrm{C} 3(\mathrm{ERG})=$ encontrar $-\mathrm{COMD}$ toro

93. y dizque abi encontró al toro.

94. semexükoj Riwo:ke seme $=x \ddot{u}=$ koj $\quad ? i=$ wo:k-e

muy $=\mathrm{EV}=$ nomás $\quad \mathrm{A} 3(\mathrm{ABS})=$ rascar $-\mathrm{INCD}$

94. Estaba nada más dizque rascando mucbo (el toro)

95. tawo:ke pu?ü

$\mathrm{ta}=$ wo:k-e pu?

$\mathrm{C} 3(\mathrm{ERG})=$ rascar-INCD arena

95. Estaba rascando la arena.

96. 2asta yukpixü?k ?ijamate pu?ü, pu?ü

Pasta yuk-pi $=x \ddot{u}=k \quad$ i $=$ jamat-e pu? pu?

hasta $\quad$ arriba- $\mathrm{LOC}=\mathrm{EV}=\mathrm{AN} \quad \mathrm{A} 3(\mathrm{ABS})=1$ legar- $\mathrm{INCD}$ arena arena

96. Hasta arriba dizque llegaba la arena, la arena.

97. Pasta yukpixü?k ?ijamate pu?ü

Pasta yuk-pi $=x \ddot{u}=k \quad \mathrm{i}=$ jamat-e $\quad$ pu?

hasta arriba- $\mathrm{LOC}=\mathrm{EV}=\mathrm{AN} \quad \mathrm{A} 3(\mathrm{ABS})=$ llegar- $\mathrm{N} \mathrm{NCD}$ arena

97. Hasta arriba dizque llegaba la arena. 

98. tana:we jama:k tu:ru 99. Piwo:ke
$\mathrm{ta}=$ na:w-e $\quad$ jamaj=k tu:ru $\mathrm{Pi}=$ wo:k-e
$\mathrm{C} 3(\mathrm{ERG})=$ botar $-\mathrm{INCD}$ aquel $=\mathrm{AN}$ toro $\quad \mathrm{A} 3(\mathrm{ABS})=$ rascar-INCD
98. La estaba aventando aquel toro. 99. Estaba rascando.
100. wo:kpa tu:ru 101. Pasta yukpi Tijamate pu?ü
wo:k-pa tu:ru Pasta yuk-pi $\mathrm{i}$ i=jamat-e pu?
rascar-INCII toro hasta arriba-LOC $\mathrm{A} 3(\mathrm{ABS})=$ llegar-INCD arena
100. Estaba rascando el toro. 101. Hasta arriba llegaba la arena.
102. nümpaxü koya
nüm-pa $=$ xü koya
decir-INCI.I=EV conejo
102. Dizque dice el conejo:
103. ?ü: japomü tiwi?k ja:mu Pinaxa?n
?ü: japo:m tüw-ik ja:mu $2 \mathrm{i}=$ nax-a?n
mmh mañana apretar- $\mathrm{PCP}$ viento $\mathrm{A} 3(\mathrm{ABS})=$ pasar-IRRD
103. -Mmb, mañana el viento va a pasar fuerte-.
104. je? Pu:raxüPk Pinümi tu:ru
je? $P u: r a=x u ̈=k \quad P i=n u ̈ m-i \quad$ tu:ru
ese hora $=\mathrm{EV}=\mathrm{AN} \quad \mathrm{A} 3(\mathrm{ABS})=$ decir-COMD toro
104. Dizque a esa bora dijo el toro:

105. jumej japom ?inaxa?n ja:mu

jumej japo:m $\mathrm{i} i=n a x-a ? n \quad$ ja:mu

cómo mañana $\mathrm{A} 3(\mathrm{ABS})=$ pasar- $\mathrm{IRRD}$ viento

105. ¿Cómo que va a pasar mañana el viento?-

106. ta taxka?tzumpa

ta $\operatorname{tax}=\mathrm{ka}:=\mathrm{tzum}-\mathrm{pa}$

COND $\mathrm{C} 1(\mathrm{LOCAL})=\mathrm{NEG}=$ amarrar-INCI.I

106. - Si no te amarro

107. mimü:nükxa:nüp ja:mu

mi=mü:-nükx-an-ü-pa ja:mu

$\mathrm{B} 2(\mathrm{ABS})=\mathrm{APL} 3$-ir-IRR-INV-INCI.I viento

107. te va a llevar el viento.

108. pero ta taxtzumpa

pero ta tax=tzum-pa

pero COND C1(LOCAL)=amarrar-INCI.I

108. Pero si te amarro 
109. mika?mü:nükxa:nüp ja:mu

$\mathrm{mi}=\mathrm{ka}:=$ üü:-nükx-an-ü-pa ja:mu

$\mathrm{B} 2(\mathrm{ABS})=\mathrm{NEG}=\mathrm{APL} 3-\mathrm{ir}-\mathrm{IRR}-\mathrm{INV}-\mathrm{INCI}$. I viento

109. no te va a llevar el viento,-

110. nümpaxü?k koya $\quad$ 111. nümpaxü?k tu:ru

nüm-pa $=x \ddot{u}=k$ koya nüm-pa $=x \ddot{u}=k$ tu:ru

decir-INCII $=\mathrm{EV}=\mathrm{AN}$ conejo decir-INCII $\mathrm{l}=\mathrm{EV}=\mathrm{AN}$ toro

110. dizque dice el conejo. 111. Dizque dice el toro:

112. tzumü?k pe?

tzum-ü-k pe?

amarrar-IMPR-INV.LOCAL de_verdad

112. -iAmárrame pues!-

113. je? ?u:rak ?ixi?k ?iyaktzumi tu:ru

je? $\quad$ u $: r a=k \quad$ Pix + ?i:y?-i=k $\quad$ Pi=yak-tzum-i tu:ru

ese hora $=\mathrm{AN}$ empezar-COMD $=\mathrm{AN} \quad \mathrm{A} 3(\mathrm{ABS})=\mathrm{PASV}$-amarrar-INCD toro

113. A esa bora el toro empezó a ser amarrado.

114. Titzumij koya

$\mathrm{P} i=$ tzum $-\mathrm{i}-\mathrm{j} \quad$ koya

$\mathrm{A} 3(\mathrm{ABS})=$ amarrar-INCD-INVD.I conejo

114. Lo estaba amarrando el conejo.

115. je? ?u:rak ?itzumiyü

je? $\quad$ u $: r a=k \quad$ i $=$ tzum-i-y

ese hora $=\mathrm{AN} \quad \mathrm{A} 3(\mathrm{ABS})=$ amarrar-COMD-INVD.C

115. A esa bora lo amarró (el conejo al toro).

116. nümpaxü?k 117. tunü paja?m

nüm-pa $=x \ddot{u}=\mathrm{k}$ tun-ü pajam

decir-INCIII $=\mathrm{EV}=\mathrm{AN}$ hacer-IMPR fuerza

116. Dizque dice (el conejo): 117. -iHaz fuerza!-
118. nümpaxü?k koya
119. tunü paja?m
120. Pe:pena
nüm-pa $=\mathrm{x} \ddot{u}=\mathrm{k}$ koya
tun-ü pajam
Pe:p-pe $=$ na
decir-INCI.I $=\mathrm{EV}=\mathrm{AN}$ conejo hacer-IMPR fuerza ver-INCI,T=todavía
118. Dizque dice el conejo: 119. --iHaz fuerza! 120. Vamos a ver
121. ta mipotpa
122. ta miwüjpa
ta $\mathrm{mi}=$ pot-pa
ta mi=wüj-pa
COND B2(ABS)=reventar-INCLII COND B2(ABS)=desatar-INCI.I
121. si revientas, 122 . si te desatas. 


123. Pe:pena 124. ta miwüjpa
Pe:p-pe=na ta mi=wüj-pa
ver-INCI.T=todavía COND B2(ABS)=desatar-INCI.I
123. Vamos a ver 124. si te desatas.

125. tunü paja?m 126. jama:k tu:ru tatuni pajamü tun-ü pajam jamaj $=\mathrm{k}$ tu:ru $\mathrm{ta}=$ tun- $\mathrm{i}$ pajam hacer-IMPR fuerza aquel=ANtoro $\mathrm{C3}(\mathrm{ERG})=$ hacer-COMD fuerza 125. -iHaz fuerza!-126. Aquel toro bizo fuerza.

$\begin{array}{ll}\text { 127. ka?wüju ja? } & \text { 128. jemak Pi?iti tzumi } \\ \text { ka:=wüj-u ja? } & \text { jem=ak } P \mathrm{i}=\text { it-i } \quad \text { alli=AN } \quad \text { A3(ABS)=existir-INCDamarrar-NMZR } \\ \text { NEG=desatar-COMI él } & \end{array}$

129. je? ?u:rak tyakpitzümi je? kuchi:nu je?k koya

je? ?u:ra=k ta=yak-pitzüm-i je? kuchi:nu je?=k koya
ese hora=AN C3(ERG)=CAUS-salir-COMD ese cuchillo ese=AN conejo
129. A esa bora sacó ese cucbillo ese conejo.

130. ?ixi?k tayo:ke

?ix $+2 \mathrm{i}: \mathrm{y} \mathrm{i}-\mathrm{i}=\mathrm{k} . \quad \mathrm{ta}=\mathrm{yo} \mathrm{k}-\mathrm{e}$

empezar-COMD $=\mathrm{AN} \quad \mathrm{C} 3(\mathrm{ERG})=$ descuartizar-INCD

130. Lo empezó a descuartizar (al toro).

131. ja:tu:runa?awak ?iyo:kpe jaj=tu:ru-na?aw=ak $\mathrm{i}=$ =o:k-pe ese=toro-AUMENT $=\mathrm{AN} \quad \mathrm{A} 3(\mathrm{ERG})=$ descuartizar-INCI.T

131. Al torote lo estaba descuartizando.

132. tyake:kayik PiPakü

$$
\begin{array}{ll}
\mathrm{ta}=\mathrm{yak}-\mathrm{ke}: \mathrm{k} 2-\mathrm{ay}-\mathrm{i}=\mathrm{k} & \mathrm{i} \mathrm{i}=\mathrm{Pak} \\
\mathrm{C} 3(\mathrm{ERG})=\mathrm{CAUS} \text {-quitar-APL2-COMD }=\mathrm{AN} & \mathrm{A} 3(\mathrm{POSD})=\text { piel } \\
\text { 132. En cuanto le quitó el cuero } &
\end{array}
$$

133. jemak tyaktiji

jem=ak ta $=$ yak-tij-i

allí=AN $\quad \mathrm{C} 3(\mathrm{ERG})=\mathrm{CAUS} \cdot$ quedar $-\mathrm{COMD}$

133. abi lo dejó.

134. jama:k koya ?ipuki?atnüpak ka:jawü mü:tak tu:ru jamaj=k koya $\mathrm{P} i=$ pük-i-Pat-nü-pe $=a \mathrm{k}$ aquel $=\mathrm{AN}$ conejo $\mathrm{A} 3(\mathrm{ERG})=$ agarrar-NMZR-considerar-ya-INCI. $\mathrm{T}=\mathrm{AN}$ 
ka:jaw mü:t=ak tu:ru

tigre $\mathrm{y}=\mathrm{AN}$ toro

134. Aquel conejo ya los traía al tigre y al toro.

135. Piwitikokü

?i=wit-i $=k 0 j=k$

$\mathrm{A} 3(\mathrm{ABS})=$ andar $-\mathrm{INCD}=$ nomás $=\mathrm{AN}$

135. Nomás caminaba

136. Piwitik jama:k koya

$\mathrm{i} i=$ wit- $\mathrm{i}=\mathrm{k} \quad$ jamaj $=\mathrm{k}$ koya

$\mathrm{A} 3(\mathrm{ABS})=$ andar $-\mathrm{INCD}=\mathrm{AN}$ aquel $=\mathrm{AN}$ conejo

136. y caminaba aquel conejo,

137. tapa:tik jama:k ?uxpi?n

$\begin{array}{ll}\mathrm{ta}=\mathrm{pa}: \mathrm{t}-\mathrm{i}=\mathrm{k} \quad \text { jamaj=k } & \text { Puxpin } \\ \mathrm{C} 3(\mathrm{ERG})=\text { encontrar } \mathrm{COMD}=\mathrm{AN} \text { aquel=AN } & \text { lagarto }\end{array}$

137. cuando encontró a aquel cocodrilo.

138. jemxük Pi?iti ?uxpi?n

$\begin{array}{lll}\mathrm{jem}=\mathrm{x} \ddot{\mathrm{u}}=\mathrm{k} & \mathrm{P} \mathrm{i}=\mathrm{Pit}-\mathrm{i} & \text { Puxpin } \\ \text { alli }=\mathrm{EV}=\mathrm{AN} & \mathrm{A} 3(\mathrm{ABS})=\text { existir-INCD } & \text { lagarto } \\ \text { 38. Abi dizque estaba el cocodrilo. } & \end{array}$

138. Ahí dizque estaba el cocodrilo.

139. semexükok jane ?ikawü:ti?

$$
\text { seme }=x u ̈=k o j=k \text { jan-e } \quad \text { i }=\text { kawüw-ti:y?-i }
$$

muy=EV=nomás=AN mentir-NMZR $\mathrm{A} 3(\mathrm{ABS})=$ cabecear-PUNTL-INCD

139. Dizque jugueteaba de a mentiras mucho con la cabeza $y$

140. jane 2i?o:yti?

jan-e $\quad$ im $=$ ?o:y-ti:y?-i

mentir-NMZR $\quad \mathrm{A} 3(\mathrm{ABS})=$ torcer-PUNTL-INCD

140. se estaba retorciendo de a mentiras.

141. nümpaxükü koya $\quad$ 142. taka:jüyta?ki?o:kpa

nüm-pa $=x \ddot{u}=k \quad$ koya $\quad$ ta $=k a:=j u ̈ y+t a: k 2 \cdot j-10: k-p a$

decir-INCII $=\mathrm{EV}=\mathrm{AN} \quad$ conejo $\mathrm{B} 1(\mathrm{ABS})=\mathrm{NEG}=$ jugar-NMZR-DESID-INCI.I

141. Dizque dice el conejo: 142. -No quiero jugar-,

143. nümpaxü ja? 144. nümpaxü?k ?uxpi?n

nüm-pa $=x \ddot{u} \quad$ ja? nüm-pa $=x \ddot{u}=k$ ?uxpin

decir-INCII $=\mathrm{EV}$ él decir-INCII $=\mathrm{EV}=\mathrm{AN}$ lagarto

143. dizque dice él. 144. Dizque dice el cocodrilo: 
$\begin{array}{ll}\text { 145. jüyta2kina } & \text { 146. nümpaxü ja? } \\ \text { jüy+ta:k2-i=na } & \text { nüm-pa=xü ja? } \\ \text { jugar-INCD=todavía } & \text { decir-INCII }=\text { EV él } \\ \text { 145. - Vamos a jugar- } & \text { 146. Dizque dice él (el cocodrilo): }\end{array}$

147. jüyta?kina pelo:ta

jüy+ta:k?-i=na pelo:ta

jugar-INCD =todavía pelota

147. - Vamos a jugar a la pelota-.

148. nümpaxü ja? ?uxpi?n 149 . ?oya 150 . nümpaxü?k koya nüm-pa $=x \ddot{u}$ ja? ?uxpin ?oya nüm-pa $=x \ddot{u}=k$ koya decir-INCI.I=EV DEF lagarto bueno decir-INCI. $=\mathrm{EV}=\mathrm{AN}$ conejo

148. Dice el cocodrilo: 149. -Está bien-150. Dizque dice el conejo:

151. ta miwampe 152. pos tajüyta?kama:t

ta $\min =w a: n ?-p e$ pos ta=jüy+ta:k?-am-a:t

COND A2(ERG) $=$ querer-INCI.T pues $B 1(\mathrm{ABS})=$ jugar-IRRI-PL.PAH

151. - Si quieres 152. pues vamos a jugar-.

153. nümpaxü ja?

nüm-pa=xü ja?

decir-INCI.I=EV él

153. Dizque dice él (el conejo).

154. ?ixi?ixü?k ?ijüyta:küxi

Pix +?i:y?-j=xü=k $\quad$ ?i=jüy+ta:k?-küx-i

empezar $-\mathrm{COMD}=\mathrm{EV}=\mathrm{AN} \quad \mathrm{A} 3(\mathrm{ABS})=$ jugar- $\mathrm{PL} 3-\mathrm{INCD}$

154. Dizque a esa bora empezaron a jugar.

155. nümpaxükü koya $\quad$ 156. jumü ya?aj ?i?iti nüm-pa $=\mathrm{x} \ddot{u}=\mathrm{k}$ koya jumü ya?aj $\mathrm{i}=$ = $\mathrm{it}-\mathrm{i}$

decir-INCII $=\mathrm{EV}=\mathrm{AN}$ conejo donde éste $\mathrm{A} 3(\mathrm{ABS})=$ existir-INCD

155. Dizque dice el conejo: 156. -iDónde está esto?

157. ta taxwopa

ta tax=wop-pa

algo $\mathrm{Cl}(\mathrm{LOCAL})=$ golpear-INCI.I

157. La parte que al golpearte

158. jumü ya?aj ta taxwopa jumü ya?aj tatax=wop-pa

donde éste algo $\mathrm{C1}(\mathrm{LOCAL})=$ golpear-INCI.I

158. ¿Dónde está esto que al golpearte 
159. jumü tike? mi?o:kanü

jumü ti?k=je? min=?o:k-a?n

donde $\quad$ CFCT=ese $A 2(A B S)=$ morir-IRRD

159. es donde te vas a morir?-

160. ta jumü ta?o:kanü

ta jumü $\tan =$ ?o:k-a?n

algo dondeA1 $(\mathrm{ABS})=$ morir- $\mathrm{RR} \mathrm{R}$

160. - iAlgo donde me muero

161. ta taxwopa?k

ta $\quad$ tax $=$ wop-pa-k

COND C1(LOCAL) $=$ golpear-INCI.I-INV.LOCAL

161. si tú me pegas?-

162. nümpaxü?k ?uxpi?n 163. jo: nümpaxü?k koya

nüm-pa $=x \ddot{u}=\mathrm{k}$ ?uxpin jo: nüm- $\mathrm{pa}=\mathrm{x} \ddot{\mathrm{u}}=\mathrm{k}$ koya

decir-INCI $\mathrm{I}=\mathrm{EV}=\mathrm{AN}$ lagarto sí decir-INCI.I=EV=AN conejo

162. dizque dice el cocodrilo. 163. -Sí-, dizque dice el conejo.

$\begin{array}{ll}\text { 164. je? tawampe } & \text { 165. xwini?a?n } \\ \text { je? } \tan =\text { wa:n2-pe } & \text { tax=wini:y?-a?n } \\ \text { ese A1(ERG)=querer-INCI.T } & \text { C1(ERG)=saber-IRR } \\ \text { 164. -Eso quiero 165. saber. } & \\ \text { 166. ta taxtzakaypa ya?aj pelo:ta } & \\ \text { ta tax=tzak-ay-pa } & \text { ya?aj pelo:ta } \\ \text { COND C1(LOCAL)=tirar-APL2-INCI.I este pelota } \\ \text { 166. Si te tiro esta pelota }\end{array}$

167. mü:t jem jumü mi?o:kanü

mü:t jem jumü $\min =$ ?o:k-a?n

y allí donde $\mathrm{A} 2(\mathrm{ABS})=$ morir-IRRD

167. $y$ abi donde te vas a morir-.

168. pos tanka?wini:pe 169. nümpaxü ja?

pos $\tan =k a$ :=wini:y?-pe nüm-pa=xü ja?

pues $\mathrm{A} 1(\mathrm{ERG})=\mathrm{NEG}=$ saber-INCI $\mathrm{T}$ decir-INCI. $\mathrm{T}=\mathrm{EV}$ él

168. -Pues no sé,-169. dizque dice él (el cocodrilo).

170. nümpaxü?k koya 171. yam tantu?stajüpü

nüm-pa $=\mathrm{xü}=\mathrm{k} \quad$ koya ya?-mü tan=tu?tz+ta-jüp

decir-INCI.I=EV=AN conejo éste-LOC $\mathrm{A} 1(\mathrm{POSD})=$ cola-nariz

170. Dizque dice el conejo: 171. -Aquí en la punta de mi cola, 
172. jeme?, ta tayakmoypa tu?k wope jem

jem=je? ta ta=yak-mo:y?-pa tuk wop-e jem

alli=ese COND B1(ABS)=PASV-dar-INCI.I uno golpear-NMZR allí

172. esa es la parte, donde si me dan un golpe alli,

$\begin{array}{clll}\text { 173. ta?o:kpa jem } & & \text { 174. nümpaxüPk koya } & \text { 175. Poya } \\ \text { ta=?o:k-pa jem } & \text { nüm-pa }=\mathrm{xü}=\mathrm{k} \text { koya } & \text { ?oya } \\ \mathrm{B} 1(\mathrm{ABS})=\text { morir-INCL.I alli } & \text { decir-INCI.I=EV=AN conejo bueno }\end{array}$

173. me muero abi-. 174. Dizque dice el conejo:175. -Está bien-.

176. nümpaxü ja? nüm-pa $=$ xü ja?

decir-INCI.I=EV él

176. Dizque dice él (el conejo):

177. taxka:tzaka?am mintu?sta?p

$$
\begin{aligned}
& \text { tax }=\mathrm{ka}:=\mathrm{tzak}-\mathrm{a} 2-\mathrm{am} \quad \min =\mathrm{tu} ? \mathrm{tz}+\mathrm{ta}-\mathrm{pi} \\
& \mathrm{C} 1(\mathrm{LOCAL})=\mathrm{NEG}=\mathrm{tirar}-\mathrm{APL} 2 \mathrm{-IRRL} 2(\mathrm{POSD})=\mathrm{cola}-\mathrm{LOC} \\
& \text { 177. }- \text { No te voy a tirar en tu cola }
\end{aligned}
$$

$\begin{array}{ll}\text { 178. jumü miPitij 179. jumü mi?o:ka?n } & \end{array}$ jumü $\min =$ ?it-i-j jumü $\min =? \mathrm{o}: \mathrm{k}-\mathrm{a}$ ?n

donde $\quad A 2(A B S)=$ existir-INCD-INVD.I donde $\quad A 2(A B S)=$ morir-IRRD

178. donde tienes 179. (la parte) donde te vas a morir-.

180. je? ?u:raxü?k koya ?ijüyta:küxi mü:tak ?uxpi?n

je? $P u: r a=x u ̈=k$ koya $? i=j u ̈ y+t a: k 2-k u ̈ x-i \quad m u ̈: t=a k$ Puxpin

ese hora $=\mathrm{EV}=\mathrm{AN}$ conejo $\mathrm{A} 3(\mathrm{ABS})=j u g a r-\mathrm{PL} 3 \cdot \mathrm{INCD}$ con $=\mathrm{AN}$ lagarto

180. Dizque a esa bora el conejo y el cocodrilo están jugando.

181. jama:k ?uxpi?n jüyta:kpa ja?

jamaj=k ?uxpin jüy+ta:k?-pa ja?

aquel=AN lagarto jugar-INCI.I él

181. Aquel cocodrilo está jugando,

182. mü: nümpaxü?k koya

mü:t nüm-pa $=x \ddot{u}=\mathrm{k} \quad$ koya

y decir-INCI. $\mathrm{I}=\mathrm{EV}=\mathrm{AN}$ conejo

182. y dizque dice el conejo:

183. ?oyamentok ya?aj taxak?o:ka?n

Poyamento $=\mathrm{k}$ ya?aj tax=yak $-? 0: \mathrm{k}-\mathrm{a} \mathrm{n}$

ahorita $=\mathrm{AN}$ éste $\mathrm{C1}(\mathrm{ERG})=$ CAUS-morir-IRRD

183. -Aborita lo voy a matar a éste-. 
184. je? ?u:raxü?k tatzakayi

.je? ?u:ra $=x u ̈=k$ ta $=$ tzak-ay-i

ese $h o r a=\mathrm{EV}=\mathrm{AN} \quad \mathrm{C} 3(\mathrm{ERG})=$ tirar-APL2-COMD

184. Dizque a esa bora le tiró (a la parte)

185. jumü Pi?o:ke je?k ?uxpi?n jumü $? \mathrm{i}=$ ?o:k-e je?=k ?uxpin

donde $\mathrm{A} 3(\mathrm{ABS})=$ morir- $\mathrm{INCD}$ ese $=\mathrm{AN}$ lagarto

185. donde muere el cocodrilo,

186. jumük ?i?o:ka?n 187. nümpaxükü

jumü $=k \quad P i=? o: k-a ? n \quad$ nüm-pa $=x \ddot{u}=k$

donde $=\mathrm{ANA}(\mathrm{ABS})=$ morir-IRRD decir-INCL. $\mathrm{I}=\mathrm{EV}=\mathrm{AN}$

186. donde se va a morir. 187. Dizque dice.

188. ?ixi? ?ijüyta:küxi

Pix+?i:y?-i $\quad$ i $i=j u ̈ y+t a: k 2-k u ̈ x-i$

empezar-COMD $\mathrm{A} 3(\mathrm{ABS})=$ jugar-PL3-INCD

188. Comenzaron a jugar.

189. ?ixi?k Pitzake

?ix + ?i:y?-i=k $\quad$ i $i=$ tzak $-\mathrm{e}$

empezar- $\mathrm{COMD}=\mathrm{AN} \mathrm{A} 3(\mathrm{ABS})=$ tirar $-\mathrm{INCD}$

189. Empezó a tirar.

190. ?initzaküxa?xej

?i=ni-tzak-küx-a?x-e-j

$\mathrm{A} 3(\mathrm{ABS})=$ RECIP-tirar-PL3-APL2-INCD-INVD.1

190. Se la tiraban,

191. Pinitzaküxa?xej jamaj pelo:ta

Pi=ni-tzak-küx-a?x-c-j jamaj pelo:ta

$\mathrm{A} 3(\mathrm{ABS})=$ RECIP-tirar-PL3-APL.2-INCD-INVD.I aquel pelota

191. y se la tiraban aquella pelota.

192. je? ?u:raxükü je?k koya tatzakayixü?k tiwi?k

je? $2 \mathrm{u}: \mathrm{ra}=\mathrm{xü}=\mathrm{k}$ je? $=\mathrm{k}$ koya ta $=$ tzak-ay- $\mathrm{i}=\mathrm{x} \ddot{\mathrm{u}}=\mathrm{k}$

ese hor $=\mathrm{EV}=\mathrm{AN}$ ese $=\mathrm{AN}$ conejo $\mathrm{C} 3(\mathrm{ERG})=$ tirar $-\mathrm{APL} 2-\mathrm{COMD}=\mathrm{EV}=\mathrm{AN}$

tüw-ik

apretar-PCP

192. A esa bora dizque el conejo le tiró duro, 
193. me:roxü?k ?itu?sjem ?itu?sjüpmü jem

$$
\text { me:ro=xü=k } \quad \mathrm{i} i=t u ? t z-j e m \quad i \mathrm{i}=\mathrm{tu} \text { ?tz-jüp-mü jem }
$$

mero $=\mathrm{EV}=\mathrm{AN} \quad \mathrm{A} 3(\mathrm{POSD})=$ cola $-\mathrm{LOC} \quad \mathrm{A} 3(\mathrm{POSD})=$ cola-nariz $\mathrm{LOC}$ alli

193. abi merito dizque en su cola, abí en la punta de la cola.

194. je? ?u:raxü?k tamoyi mü:t je? pelo:ta

$$
\text { je? } ? \mathrm{u}: \mathrm{ra}=\mathrm{xü}=\mathrm{k} \text { ta }=\text { mo:y? } \mathrm{i} \quad \text { mü:t je? pelo:ta }
$$

ese hora $=\mathrm{EV}=\mathrm{AN} \quad \mathrm{C} 3(\mathrm{ERG})=\mathrm{dar}-\mathrm{COMD}$ con ese pelota

194. A esa bora dizque le dió con esa pelota.

195. tatojwopi

$$
\begin{aligned}
& \mathrm{ta}=\text { toj-wop- } \mathrm{i} \\
& \mathrm{C} 3(\mathrm{ERG})=\mathrm{APL} 4 \text {-golpear-COMD }
\end{aligned}
$$

195. Lo golpeó.

196. je? ?u:raxü?k ?i?o:ki je?k ?uxpi?n

$$
\begin{aligned}
& \text { je? } 2 \mathrm{u}: \mathrm{ra}=\mathrm{xü}=\mathrm{k} \quad \mathrm{i}=20: \mathrm{k}-\mathrm{i} \quad \mathrm{je}=\mathrm{k} \quad \text { Puxpin } \\
& \text { ese hora }=\mathrm{EV}=\mathrm{AN} \quad \mathrm{A}(\mathrm{ABS})=\text { morir-COMD ese }=\mathrm{AN} \text { lagarto } \\
& \text { 196. A esa bora dizque se murió ese cocodrilo. }
\end{aligned}
$$

197. 2o:kuxü?k jem

2o:k-u $=x u ̈=k \quad j e m$

morir- $\mathrm{COMI}=\mathrm{EV}=\mathrm{AN}$ allí

197. Dizque se murió ahí.

198. je?k Puxpi?n jem jumü Pipüjni?

je?=k ?uxpin jem jumü ?i=püj-ni:y?-i

ese $=$ AN lagarto allí donde $\mathrm{A} 3(\mathrm{ABS})=$ reventar-DIR:sin.mov-COMD

198. Ese cocodrilo abi donde quedó tiradote con la panza para arriba.

199. je? ?u:raxü?k koya tani:yo:ki ?uxpi?n

$$
\text { je? ?u:ra }=x \ddot{u}=\mathrm{k} \text { koya ta=ni:-yo:k-i 2.. Tuxpin }
$$

ese hora $=\mathrm{EV}=\mathrm{AN}$ conejo $\mathrm{C} 3(\mathrm{ERG})=$ SUPERF-descuartizar-COMD lagarto

199. Dizque a esa bora el conejo descuartizó al cocodrilo.

200. tyakpitzümayik ?i?akü

$$
\begin{array}{ll}
\text { ta }=\text { yak-pitzüm-ay- } \mathrm{i}=\mathrm{k} & \mathrm{i}=\mathrm{Pak} \\
\mathrm{C} 3 \text { (ERG) }=\text { CAUS-salir-APL2-COMD=AN } & \mathrm{A} 3(\mathrm{POSD})=\text { piel }
\end{array}
$$

200. Le quitó el cuero.

201. tuwük ka:jawakü mü:tak tu:ru mü:tak ?uxpi?n tuwük ka:jaw=ak mü:t=ak tu:ru mü:t=ak ?uxpin tres tigre $=\mathrm{AN}$ con $=\mathrm{AN}$ toro $\mathrm{con}=\mathrm{AN}$ lagarto 201. Son tres junto con el tigre, el toro y el cocodrilo. 
202. tuwükak tamü:nükxno

tuwük=ak ta=mü:-nükx-nü-e

tres $=\mathrm{AN} \quad \mathrm{C} 3(\mathrm{ERG})=\mathrm{APL} 3-\mathrm{ir}-\mathrm{ya}-\mathrm{INCD}$

202. Ya llevaba a los tres.

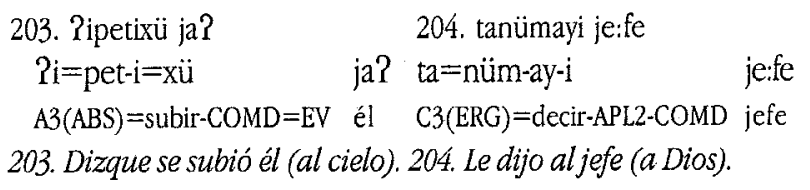

205. ?iyaj taxmü:minayu ja?

?iyaj tax=mü:-mi:n?-ay-u ja?

aquí $\quad \mathrm{C} 1($ LOCAL) $=\mathrm{APL} 3$-venir-APL2-COMI él

205. -Aqui te los traje.

206. tamü:minküxu ja?

207. Poya

$\tan =$ mü:-mi:n?-küx-u

ja? ?oya

$\mathrm{A} 1(\mathrm{ERG})=\mathrm{APL} 3$-venir-PL3-COMI él bueno

206. Los traje- 207 -Está bien-,

208. nümpaxü?k je:fe 209. nümpaxü?k

nüm-pa $=x \ddot{u}=k \quad$ je:fe nüm-pa $=x u ̈=k$

decir-INCI.I $=\mathrm{EV}=\mathrm{AN} \quad$ jefe $\quad$ decir-INCI. $\mathrm{I}=\mathrm{EV}=\mathrm{AN}$

208. dizque dice el jefe (Dios), 209. Dizque dice:

210. michu:chuchikna? seme

$\mathrm{mi}=\mathrm{chu}$ :chu-chik-na? seme

$\mathrm{B} 2(\mathrm{ABS})=$ chico-DIM-ADVZR muy

210. - Eres muy cbiquitito.

211. je? ti?k ta?nü?k mi?itanü

je? ti?k ta?nük min=?it-a?n

ese CFCT grande $A 2(A B S)=$ existir-IRRD

211. Eso si fueras grande,

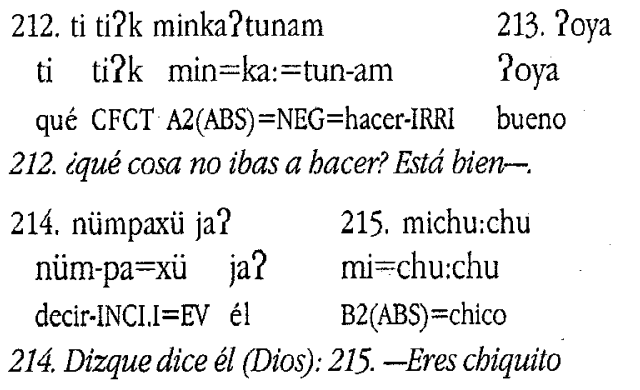




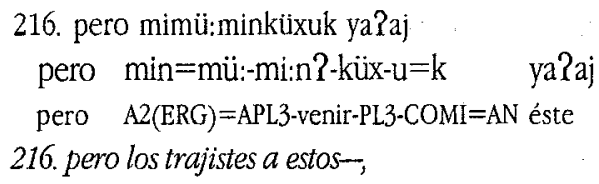

217. nümpaxü ja? 218, ma:sak ti?k ta?nü?k mi?itanü nüm-pa=xü ja? ma:s=ak ti?k ta?nük min=?it-a?n decir-INCIII $=\mathrm{EV}$ él más=ANCFCT grande $\mathrm{A} 2(\mathrm{ABS})=$ existir-IRRD

217. dizque dice él (Dios). 218. -Si fueras más grande

219. ti ti?k minka?tunam

$$
\begin{array}{lll}
\text { ti } & \text { tîk } & \min =\mathrm{ka}:=\text { tun-am } \\
\text { qué } & \mathrm{CFCT} & \mathrm{A} 2(\mathrm{ABS})=\mathrm{NEG}=\text { hacer-IRRI }
\end{array}
$$

219. ¿qué cosa no ibas a bacer?-

220. je? ?u:raxü?k ?iyakchipkota?xiy ja?xü Pita:tzükü

$$
\begin{aligned}
& \text { je? } \quad \text { ?u:ra }=x u ̈=k \quad \text { ? } i=y a k-c h i p-k o t-a ? x-i-y \\
& \text { ese hora }=\mathrm{EV}=\mathrm{AN} \quad \mathrm{A} 3(\mathrm{ABS})=\mathrm{PASV} \text {-aruñar-juntar-APL2-COMD-INVD.C } \\
& \text { ja? }=\text { xü } \mathrm{Q} i=\text { ta:tzük } \\
& \text { él=EV A3(POSD) }=\text { oreja }
\end{aligned}
$$

221. ?ixi?ixük ?ija:yakwititij mü:t ?ita:tzü?k

$$
\begin{array}{ll}
2 \mathrm{ix}+2 \mathrm{i}: \mathrm{y} 2-\mathrm{i}=\mathrm{x} \ddot{\mathrm{u}}=\mathrm{k} & \mathrm{i} \mathrm{i}=\mathrm{ja}:=\text { ak }- \text { witit- } \mathrm{i}-\mathrm{j} \\
\text { empezar-COMD }=\mathrm{EV}=\mathrm{AN} & \mathrm{A} 3(\mathrm{ABS})=\text { MIRAT=CAUS-girar-INCD-INVD. } \mathrm{I}
\end{array}
$$$$
\text { mü:t } \quad \text { i }=\text { ta:tzük }
$$

con $\mathrm{A} 3(\mathrm{POSD})=$ oreja

221. (Dios) le empieza a darle vueltas (al conejo) agarrándolo de su oreja.

222. je? ?u:raxüik koya ?asta wepxü?k ?ika?i

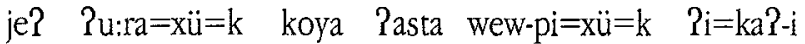

$$
\begin{aligned}
& \text { ese hora }=\mathrm{EV}=\mathrm{AN} \text { conejo hasta allá-LOC }=\mathrm{EV}=\mathrm{AN} \quad \mathrm{A} 3(\mathrm{ABS})=\text { bajar }-\mathrm{COMD} \\
& \text { 222. Dizque a esa bora el conejo dizque basta allá cayó. }
\end{aligned}
$$

223. je? ?u:raxüip ?iye:ki ma:s ?ita:tzü?k

$$
\begin{aligned}
& \text { je? } P u: r a=x u ̈=k \text { Pil=ye:k-i ma:s } P i=t a: t z u ̈ k \\
& \text { ese hora }=\mathrm{EV}=\mathrm{AN} \quad \mathrm{A} 3(\mathrm{ABS})=\text { crecer }-\mathrm{COMD} \text { más } \quad \mathrm{A} 3(\mathrm{POSD})=\text { oreja }
\end{aligned}
$$

223. Dizque a esa bora le creció más su oreja.

224. ta:tzüPk koyana?aw ye:kuk ma:s ?ita:tzüik

$$
\begin{aligned}
& \text { ta: tzük koya-na?aw ye:k-u=k ma:s } \mathrm{P}=\mathrm{i}=\mathrm{ta}: \mathrm{tzük} \\
& \text { oreja conejo-AUMENT crecer-COMI=AN más } \mathrm{A} 3(\mathrm{POSD})=0 \text { reja } \\
& \text { 224. Al conejo orejón le creció más su oreja. }
\end{aligned}
$$


1. ?ituxü tu?k luga?r

?it-u=xü tuk lugar

existir-COMI=EV uno lugar

1. Dizque bubo un lugar.

2. ja?xü Pamü jatpa ?en tukxü xa:patu

ja?=xü ?am jat-pa ?en tuk=xü xa:patu

$\mathrm{DEF}=\mathrm{EV}$ huapango suceder-INCI.I en uno $=\mathrm{EV}$ sábado

2. Dizque babia buapango en un sábado.

3. jamaxü ju?kupakü ni?tik xa:patu mayexü?k tatunküxi ?amü jamaj=xü ju?kopak ni?tik xa:patu maye $=x \ddot{u}=k$

aquel $=\mathrm{EV}$ pueblo todo sábado TEMP $=\mathrm{EV}=\mathrm{AN}$

ta $=$ tun-küx-i ?am

$\mathrm{C} 3(\mathrm{ERG})=$ hacer-PL $3 \cdot \mathrm{INCD}$ huapango

3. Dizque en aquel pueblo todos los sábados hacian buapango.

4. xa:patu mayexü?k tatunküxi ?amü

xa:patu maye $=x \ddot{u}=k$ ta $=$ tun-küx-i?am

sábado $\quad \mathrm{TEMP}=\mathrm{EV}=\mathrm{AN} \quad \mathrm{C} 3(\mathrm{ERG})=$ hacer-PL3-INCD huapango

4. Dizque todos los sábados bacian buapango

5. mü:takü jamaj jaykakü ?i?e:pküxpexü?k

mü:t=ak jamaj jaykak ?i=?e:p-küx-pe $=x u ̈=k$

$\mathrm{y}=\mathrm{AN}$ aquel gente $\mathrm{A} 3(\mathrm{ERG})=\mathrm{ver} \cdot \mathrm{PL} 3-\mathrm{INCI}, \mathrm{T}=\mathrm{EV}=\mathrm{AN}$

5. y aquella gente dizque miraba

6. ?ijamate tu?k

?i=jamat-e tuk

$\mathrm{A} 3(\mathrm{ABS})=1$ legar-INCD uno

6. que llegaba uno,

7. xa:patu mayexü?k ?ijamate Pespa?

xa:patu maye $=x \ddot{u}=\mathrm{k} \quad$ ?i=jamate $\quad$ Retz-pa-?

sábado $\quad T E M P=E V=A N \quad A 3(A B S)=$ llegar-INCD bailar-INCI.I-NMZR

7. que todos los sábados llegaba a bailar.

8. na:xe:xü ?ijate ?amü

na?kxej=xü ?i=jat-e ?am

cuando $=\mathrm{EV} \quad \mathrm{A} 3(\mathrm{ABS})=$ suceder $-\mathrm{INCD}$ huapango

8. Dizque cuando bacian buapango 
9. ka?faya:ttumpaxü ja?

ka:=faya:r-tun-pa $=x \ddot{u} \quad$ ja?

$\mathrm{NEG}=$ fallar-hacer-INCI.I=EV él

9. no fallaba.

10. sye:mpre na:xe:xü ?i?iti ?amü

sye:mpre na?kxej=xü $2 \mathrm{i}=$ ?it-i $\quad$ ?am

siempre cuando $=\mathrm{EV} \quad \mathrm{A} 3(\mathrm{ABS})=$ existir-INCD huapango

10. Siempre dizque cuando habia buapango,

11. jemxü?k ?ijamate tukxük yojwa

jem $=x \ddot{u}=k \quad$ ?i=jamate $\quad$ tuk $=x \ddot{u}=k$ yo? jwa

allí $=\mathrm{EV}=\mathrm{AN} \quad \mathrm{A} 3(\mathrm{ABS})=$ llegar- $\mathrm{INCD} \quad$ uno $=\mathrm{EV}=\mathrm{AN}$ hombre

11. dizque abi llegaba un bombre.

12. pero jamaj jaykakü Pika?ixkape ja?

pero jamaj jaykak $\mathrm{P} i=\mathrm{ka}=$ : $\mathrm{ix}+\mathrm{kap}$-pe $\quad \mathrm{ja}$ ?

pero aquel gente $\mathrm{A} 3(\mathrm{ERG})=\mathrm{NEG}=$ conocer-INCI. $T$ él

12. Pero aquella gente no sabía

13. püna:je? 14. mü:tü Pasta Piküxixü ?amü

pün-?aj=je? mü:t Rasta $2 \mathrm{i}=$ küx-i=xü $\quad$ Pam

quién-RLTVZR=ese $y \quad$ hasta $A 3(\mathrm{ABS})=$ terminar-INCD $=E V$ huapango

13. quién era ese. 14. Y dizque basta que terminaba el buapango

15. ?inükxnoxü ja?

?i=nükx-nü-i=xü ja?

$\mathrm{A} 3(\mathrm{ABS})=\mathrm{ir}-\mathrm{ya}-\mathrm{INCD}=\mathrm{EV}$ él

15. entonces ya dizque se iba él.

16. Pasta ?iküxnoxü ?amü

Pasta $P \mathrm{i}=$ küx-nü-i=xü Pam

hasta $\mathrm{A} 3(\mathrm{ABS})=$ terminar-ya- $\mathrm{INCD}=\mathrm{EV}$ huapango

16. Hasta que ya se acababa el buapango

17. Pinükxno ja?

?i=nükx-nü-e ja?

$A 3(A B S)=i r-y a-I N C D$ él

17. ya se iba él.

18. mü:t jayka?k ?ika?ixkape

mü:t jaykak $\mathrm{i} i=\mathrm{ka}:=\mathrm{ix}+\mathrm{kap}-\mathrm{pe}$

y gente $\mathrm{A} 3(\mathrm{ERG})=\mathrm{NEG}=$ conocer-INCI $\mathrm{T}$

18. Y la gente no lo conocía. 
19. mü:t jamaj jayka?k ?ika?ixkape

mü:t jamaj jaykak $\mathrm{R} i=\mathrm{ka}=\mathrm{Pix}+\mathrm{kap}$-pe

y aquel gente $\mathrm{A} 3(\mathrm{ERG})=\mathrm{NEG}=$ conocer-INCI.T

19. Y aquella gente no lo conocia,

20. pero ?ijamatixü $? \mathrm{u}:$ :ra

pero $2 \mathrm{i}=$ jamat- $\mathrm{i}=\mathrm{xü} \quad$ Pu:ra

pero $\mathrm{A} 3(\mathrm{ABS})=$ llegar $-\mathrm{COMD}=\mathrm{EV}$ hora

20. pero dizque llegó la bora

21. ?ixi?ixü?k ?inünküxi ?entre je?tü?k

Pix+?i:y?-i=xü=k ?i=nüm-küx-i $\quad$ Rentre je?-tük

empezar-COMD $=\mathrm{EV}=\mathrm{AN} \quad \mathrm{A} 3(\mathrm{ABS})=$ decir-PL3-INCD entre ese-PL

21. en que comenzaron a decirse entre ellos

22. pün ya?jü 23. tanka?ixkape:ta?

pün ya?aj $\tan =k a:=? i x+k a p-p e-: t=a ?$

quién éste $\mathrm{A}(\mathrm{ERG})=\mathrm{NEG}=$ conocer-INCI.T.PL.PAH $=\mathrm{AN}$

22. ¿Quién es éste? 23. No lo conocemos.

24. pün?atam

pün-?at-am

quién-considerar-IRRI

24. ¿Quién será?

25. porke ka:da we?s ?ijate ?amü

porke ka:da wes $2 \mathrm{i}=$ jat-e

Pam

porque cada vez $A 3(\mathrm{ABS})=$ suceder-INCD huapango

25. Porque cada vez que bay buapango

26. mimpa ja?

mi:n?-pa ja?

venir-INCI.I él

26. viene él.

27. tej pe? ti: tantuname:t

te?ej pe? ti: tan=tun-am-e:t

ahora de_verdad qué Al(ERG)=hacer-IRRI-PL.PAH

27. Yabora iqué vamos a hacer?-

28. ?ikapxküxi ?entre je?tü?k

?i=kapx-küx-i Pentre je?-tük

$A 3(A B S)=h a b l a r-P L 3-I N C D$ entre ese-PL

28. Se estaban bablando entre ellos, 
29. Piyakni?ampiwküxij

Pi=yak-ni-Pampiw-küx-i-j

$\mathrm{A} 3(\mathrm{ABS})=\mathrm{CAUS}-\mathrm{RECIP}-$ platicar-PL3-INCD-INVD.I

29. Se estaban platicando.

30. posü nümpaxï ja:tukaj

pos nüm-pa=xü ja:-tuk-?aj

pues decir-INCI.I =EV otro-uno-RLIVZR

30. Pues dizque dice el otro:

31. tajuyame:t tu?k warri?l Pampanü:jü

$\tan =$ juy-am-e:t tuk warril Ran+pa-nü:

$\mathrm{A} 1$ (ERG) $=$ comprar-IRRI-PL.PAH uno barril ardiente-agua

31. -Vamos a comprar un barril de aguardiente

32. mü:tak xyak?u:ka?ne:t

mü:t=ak tax=yak-?u:k-a?n-e:t

$y=A N \quad C 1(E R G)=$ CAUS-tomar-IRRD-PL.PAH

32. y le vamos a dar de tomar-.

33. Roya nümpaxi ja? 34. ?ijamatixü xa:patu

?oya nüm-pa=xü ja? $\mathrm{i} i=j a m a t-i=x \ddot{u} \quad$ xa:patu

bueno decir-INCl.I=EV él $\mathrm{A} 3(\mathrm{ABS})=$ llegar-COMD $=\mathrm{EV}$ sábado

33. - Está bien-, dizque dice él. 34. Dizque llegó el sábado

35. Ri?ixiyixü Pamü

$\mathrm{Pi}=2 \mathrm{ix}+\mathrm{Pi}: \mathrm{y}$ - $\mathrm{i}=\mathrm{x} \ddot{\mathrm{i}} \quad$ ?am

$\mathrm{A} 3(\mathrm{ABS})=$ empezar $-\mathrm{COMD}=\mathrm{EV}$ huapango

35. y dizque comenzó el buapango,

36. Pixiyixü Pi?etze jayka?k

Pix+?i:y2-i=xü $\quad 2 \mathrm{i}=$ ?etz-e jaykak

empezar-COMD $=E V \quad A 3(A B S)=$ bailar-INCD gente

36. y dizque empezó a bailar la gente,

37. mü:tak jama:k yojwa jamatpa?kojü

mü:t=ak jamaj=k yo?jwa jamat-pa-a?=koj

$\mathrm{y}=\mathrm{AN} \quad$ aquel=AN hombre llegar-INCI.I-RLTVZR=nomás

37. y aquel bombre, el que llegaba nomás,

38. Pespaxü?k 39. PiPetze

?etz-pa $=x \ddot{u}=k \quad$ i $=$ Petz-e

bailar- $\mathrm{INCI}, \mathrm{I}=\mathrm{EV}=\mathrm{AN} \quad \mathrm{A} 3(\mathrm{ABS})=$ bailar $-\mathrm{INCD}$

38. dizque baile, 39. ybaile. 
40. je? jamatpak Respa? ?amjem

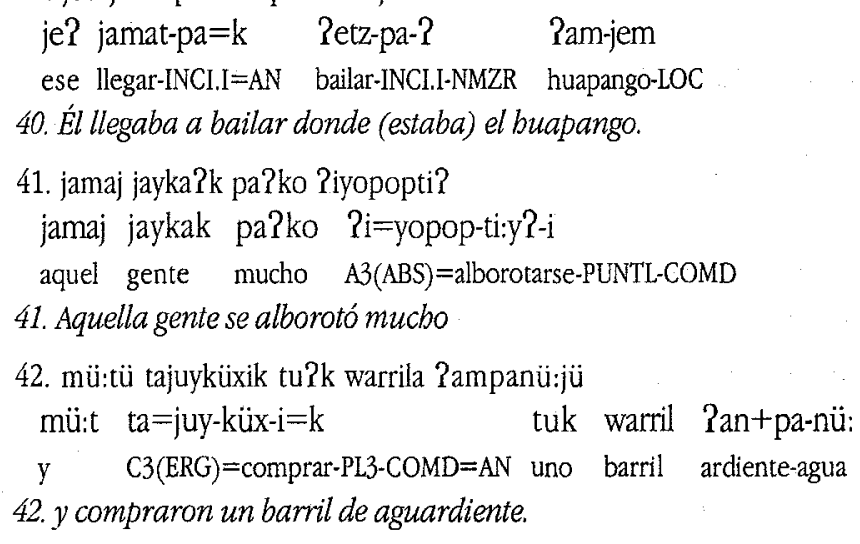

42. mü:tü tajuyküxik tu?k warrila ?ampanü:jü

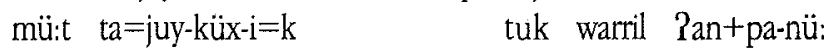

48. ta juyküxi tu?k warri?l ?ampanü:jü ta=juy-küx-i tuk warril ?an+pa-nü: $\mathrm{C} 3(\mathrm{ERG})=$ comprar-PL3-COMD uno barril ardiente-agua 48. Compraron un barril de aguardiente. 
49. jemxü?k tachikxküxno tu?k warri?! ?ampanü:jü jem $=x u ̈=k$ ta $=$ chikx-küx $-n \ddot{n}-\mathrm{i} \quad$ tuk warril alli $=\mathrm{EV}=\mathrm{AN} \quad \mathrm{C3}(\mathrm{ERG})=$ tener_bajo_cuidado-PL3-ya-INCD uno barril ?an+pa-nü:

ardiente-agua

49. Abi ya tenian ellos un barril de aguardiente.

50. 2al ?ijamatikü

Ral $2 \mathrm{i}=$ jamat-i $=\mathrm{k}$

al $\mathrm{A} 3(\mathrm{ABS})=$ llegar $-\mathrm{COMD}=\mathrm{AN}$

50. Cuando llegó (el diablo)

51. 2ipetik jem na?kajem

? $\mathrm{i}=$ pet $\mathrm{i}=\mathrm{k} \quad \mathrm{jem}$ na?ka-jem

$\mathrm{A} 3(\mathrm{ABS})=$ subir- $\mathrm{COMD}=\mathrm{AN}$ allí tabia- $\mathrm{L} O \mathrm{C}$

51. se subió abí en la tarima.

52. jata ?ipetixü ja?

jata $\quad$ i $i=$ pet- $\mathrm{i}=\mathrm{xü} \quad \mathrm{ja}$ ?

enseguida $\mathrm{A} 3(\mathrm{ABS})=$ subir- $\mathrm{COMD}=\mathrm{EV}$ él

52. Enseguida dizque se subió,

\section{Pixi?ixü?k Pi?etze 54. Pi?etze \\ Pix + Pi:y?-i=xü=k $\quad$ i $=$ ?etz-e $\quad$ ?i=?etz-e}

empezar $-\mathrm{COMD}=\mathrm{EV}=\mathrm{AN} \mathrm{A} 3(\mathrm{ABS})=$ bailar $-\mathrm{INCD} \mathrm{A} 3(\mathrm{ABS})=$ bailar $-\mathrm{INCD}$

53. y empezó a bailar 54. y a bailar,

55. jama:kü Rimo?a:me?e Pampanü:ji

$$
\text { jamaj=ak } \quad \mathrm{i}=\text { =mo:yi-am-e? } \quad \text { Pan+pa-nü: }
$$

aquel $=\mathrm{AN} \quad \mathrm{A} 3(\mathrm{ERG})=\mathrm{dar}-\mathrm{IRR}-\mathrm{RLTVZR}$ ardiente-agua

55. aquel al que le van a dar aguardiente.

56. jemak tachikxno ja?xü tukxü chima

$$
\text { jem }=\text { ak ta }=\text { chikx-nü-e ja? }=x u ̈ \text { tuk=xü chima }
$$

alli $=\mathrm{AN} \quad \mathrm{C} 3(\mathrm{ERG})=$ tener_bajo_cuidado-ya-INCD él $=\mathrm{EV} \quad$ uno $=\mathrm{EV}$ jicara

56. Abi él dizque ya tenía una jicara.

57. je? ?u:taxükü taje:pi je? ?ampanü:jü
je? $\quad$ iu: $r a=x u ̈=k$ ta $=j e: p-i$
je? ?an+pa-nü:
ese hora $=\mathrm{EV}=\mathrm{AN} \quad \mathrm{C}(\mathrm{ERG})=$ pescar $-\mathrm{COMD}$ ese ardiente-agua

57. A esa bora le sirvieron (con un recipiente bueco) ese aguardiente. 
58. tamoyixü ja?

ta $=$ mo:y?-i=xü $\quad j a ?$

$\mathrm{C} 3(\mathrm{ERG})=\mathrm{dar}-\mathrm{COMD}=\mathrm{EV}$ él

58. Ellos dizque se lo dieron.

59. je? ?u:raxü?k ta?u:ki je?k ko?yaj je? ?ampanü:jü

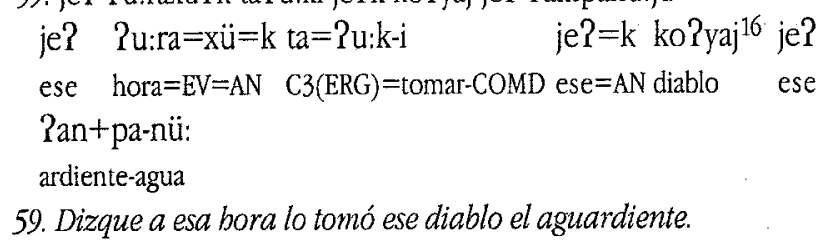

59. Dizque a esa bora lo tomó ese diablo el aguardiente.

60. pero tu?k chima, ja?mexükoj ka:da rratuxü?k tamoyküxi pero tuk chima ja?mej=xü=koj ka:da $\quad$ ratu $=x \ddot{u}=k$

pero uno jícara asi $=\mathrm{EV}=$ nomás cada rato $=\mathrm{EV}=\mathrm{AN}$

ta $=$ mo:y?-küx-i

$\mathrm{C} 3(\mathrm{ERG})=\mathrm{dar}-\mathrm{PL} 3-\mathrm{INCD}$

60. Pero una jicara, dizque asi nomás cada rato le daban.

61. ka:da rratuxü?k jamaxü?ü warrilü ka?nüpxü?

ka:da rratu $=x \ddot{u}=k$ jamaj $=x u ̈$ warril ka?-nü-pa $=x \ddot{~}$

cada rato $=\mathrm{EV}=\mathrm{AN}$ aquel $=\mathrm{EV}$ barril bajar-ya-INCI.I=EV

61. Dizque a cada rato, dizque aquel barril dizque ya estaba bajando.

62. Pixküjpu:txü Pinükxi del warri?l

$$
\begin{array}{ll}
\text { Pixküj+pu:t=xü } & \text { Pi=nükx-i delwarril } \\
\text { mitad=EV } & A 3(A B S)=i r-C O M D \text { del barril }
\end{array}
$$

62. Dizque la mitad del barril se terminó.

63. jama:k yojwa, ko?yajü, tiwikak ?i?etze

$$
\begin{array}{llll}
\text { jamaj=k yo?jwa } & \text { ko?yaj } & \text { tüw- } \mathrm{ik}=\mathrm{ak} & \mathrm{i}=\text { ?etz-e } \\
\text { aquel=AN hombre } & \text { diablo } & \text { apretar- } \mathrm{PCP}=\mathrm{AN} & \mathrm{A} 3(\mathrm{ABS})=\text { bailar }-\mathrm{INCD}
\end{array}
$$

63. Y aquel bombre, el diablo, bailaba duro.

$\begin{array}{ll}\text { 64. Petzpa ja? } & \text { 65. Pipojti? } \\ \text { Petz-pa ja? } & \text { Pi=poj-ti:y?-i } \\ \text { bailar-INCI.I él } & \text { A3(ABS)=patear-PUNTL-INCD }\end{array}$

64. Bailaba, 65. zapateaba.

\footnotetext{
${ }^{16}$ La combinación de morfemas que dio lugar al sustantivo ko?yaj 'diablo' es la siguiente: ka:'negación', Poya 'bueno', - $2 a j$ 'relativizador'.
} 
66. ja?mexükoj ja?mexükoj tamoyküxi

$$
\begin{aligned}
& \text { ja?mej=xü=koj ja?mej=xü=koj ta=mo:y?-küx-i } \\
& \text { asi }=\mathrm{EV}=\text { nomás } \quad \text { asi }=\mathrm{EV}=\text { nomás } \quad \mathrm{C} 3(\mathrm{ERG})=\text { dar-PL3-INCD }
\end{aligned}
$$

66. Dizque así nomás, dizque así nomás le daban.

67. pero por chima ?u:kpa ja?

$$
\text { pero por chima ?u:k-pa ja? }
$$$$
\text { pero por jícara tomar-INCI.I él }
$$

\section{Pero tomaba a jicarazos.}

68. me:roxü Piküxno me:ro=xü Pi $=$ küx-nü-e mero=EV $\mathrm{A} 3(\mathrm{ABS})=$ terminar-ya-INCD

68. Dizque ya mero se estaba acabando.

69. je? ?u:raxü?k ?ipoyi

$$
\begin{array}{lll}
\text { je? } & \text { Pu:ra }=x \ddot{u}=k & 2 i=\text { po: } y 2-i \\
\text { ese } & \text { hora }=E V=A N & A 3(A B S)=h u i r-C O M D
\end{array}
$$

69. Dizque a esa bora se buyó.

70. pero li:sto jamaj ?i?itno ni?ti?k küpi

$$
\begin{array}{lll}
\text { pero li:sto jamaj } & \text { Pi=?it-nü-e } & \text { ni?tik küpi } \\
\text { pero listo aquel } & A 3(A B S)=\text { existir-ya-INCD } & \text { todo leña }
\end{array}
$$

70. Pero ya estaba lista toda esa leña.
71. küpi li:sto Pi?itno 72. ka?tze?k
küpi li:sto ?i=?it-nü-e ka?tz-ek

leña listo $\mathrm{A} 3(\mathrm{ABS})=$ existir-ya-INCD cortar-PCP

71. La leña ya estaba lista. 72. Estaba cortada en trozos (los cuales iban a servir)

73. para tyaktoyküxa?nxü ja?

$$
\text { para ta=yak-toy-küx-a?n=xü ja? }
$$

para C3(ERG) $=$ CAUS-quemarse-PL3-IRRD $=E V$ él

73. para que dizque lo quemaran a él (al diablo).

74. mü:tii je? ?u:rak ?iyü?knejü

mü:t je? ?u:ra=k ?i=yü?k-nü-i

Y ese hora $=\mathrm{AN} \quad \mathrm{A} 3(\mathrm{ABS})=$ salir-ya-COMD

74. Ya esa bora (el diablo) ya se salió (del baile),

75. mu:künüpa? 76. mu:künüpa? mu:k-nü-ü-pa $=\mathrm{a} ? \quad$ mu:k-nü-ü-pa $=\mathrm{a}$ ? chupar-ya-INV-INCI.I=AN chupar-ya-INV-INCI.I=AN

75. ya borracbo, 76. ya borracho. 
77. je? ?u:rak ?ipeti ?ikawa:yu?p

$$
\begin{aligned}
& \text { je? } \text { ?u } r a=k \text { ?i=pet-i } \quad \text { i } i=k a w a: y u-p i \\
& \text { ese hora }=\mathrm{AN} \quad \mathrm{A} 3(\mathrm{ABS})=\text { subir }-\mathrm{COMD} \quad \mathrm{A} 3(\mathrm{POSD})=\text { caballo- } \mathrm{LOC}
\end{aligned}
$$

78. pero jamaj jaykakü tu?awpu:t ?i?itno

$$
\begin{aligned}
& \text { pero jamaj jaykak tu?aw-pu:t } \text { i }=\text { ?it-nü-e } \\
& \text { pero aquel } \text { gente camino-mitad } \mathrm{A} 3(\mathrm{ABS})=\text { existir-ya-INCD } \\
& \text { 78. pero aquella gente ya estaba en medio del camino. }
\end{aligned}
$$

$$
\begin{array}{cl}
\text { 79. ni?ti?k ta?a:wixküxnok } & \text { 80. Pinaxa?n } \\
\text { ni?tik ta }=\text { ?a:wix-küx-nü-i=k } & \text { ?i=nax-a?n }
\end{array}
$$$$
\text { todo } \mathrm{C} 3(\mathrm{ERG})=\text { esperar-PL3-ya-INCD }=\mathrm{AN} A 3(\mathrm{ABS})=\text { pasar }-\mathrm{IRRD}
$$

79. Todos ya lo estaban esperando 80. a que pasara.

81. je? ?u:raxü?k ?inaxexü?k

$$
\begin{array}{lll}
\text { je? } & \text { Pu:ra }=\mathrm{xu}=\mathrm{k} & 2 \mathrm{i}=\text { nax-e }=\mathrm{xu}=\mathrm{k} \\
\text { ese } & \text { hora }=\mathrm{EV}=\mathrm{AN} & \mathrm{A} 3(\mathrm{ABS})=\text { pasar }-\mathrm{INCD}=\mathrm{EV}=\mathrm{AN}
\end{array}
$$

81. Dizque a esa bora dizque iba pasando.

82. mikitmikitna? kawa:yu?mak ?imi:no

$$
\text { mikit-mikit-na? kawa:yu-mü=ak } \quad \text { ie=mi:n?-nü-i }
$$

a_tropel-a $\_$tropel-ADVZR caballo-LOC $=\mathrm{AN} \quad \mathrm{A} 3(\mathrm{ABS})=$ venir-ya-INCD

82. Ya venía corriendo a tropel arriba del caballo.

83. mü:t jamaj jaykakü ko:chikxnüpa?aj tu?awjemü

$$
\text { mü:t jamaj jaykak ko:+chikx-nü-pa-Paj tu?aw-jem }
$$

y aquel gente cuidar-ya-INCII-RLTVZR camino-IOC

83. Y aquella gente ya estaba en espera de él en el camino

84. je? ?u:rak Pinaxi je? na?awa:kü

$$
\begin{aligned}
& \text { je? } \quad \mathrm{u}: \mathrm{ra}=\mathrm{k} \text { ? } \mathrm{i}=\text { nax }-\mathrm{i} \quad \mathrm{je} \text { na?aw }=\mathrm{ak} \\
& \text { ese hora }=A N \quad A 3(A B S)=\text { pasar-COMD ese anciano=AN }
\end{aligned}
$$

84. A esa bora pasó ese grandote (el diablo).

85. je? ?u:rak ?ixi?k ?ipiyü?küxi ?ijayjü?na de je?k ko?yaj

$$
\text { je? ?u:ra }=\mathrm{k} \text { ?ix }+ \text { ?i:y?-i=k } \quad \text { i } \mathrm{i}=\text { piyü?k-küx-i }
$$$$
\text { ese hora }=\mathrm{AN} \text { empezar }-\mathrm{COMD}=\mathrm{AN} \quad \mathrm{A} 3(\mathrm{ABS})=\text { correr }-\mathrm{PL} 3-\mathrm{COMD}
$$$$
\mathrm{i}=\mathrm{jay}-\mathrm{jü} \text { ?na de } \mathrm{je} \mathrm{i}=\mathrm{k} \text { ko?yaj }
$$$$
\mathrm{A} 3(\mathrm{POSD})=\text { ajeno-atrás de ese }=\mathrm{AN} \text { diablo }
$$

85. A esa bora empezaron a correr por atrás de ese diablo. 
86. jayka?k 2imi?n piyü?kpa ?ijü?na?p

jaykak $2 \mathrm{i}=$ mi:n?-i $\quad$ piyü?k-pa $\mathrm{i} i=$ jü?na-pi

gente $\mathrm{A} 3(\mathrm{ABS})=$ venir-INCD correr-INCI.I $\quad \mathrm{A} 3(\mathrm{ABS})=$ atrás-LOC

86. Esa gente venía corriendo atrás de él.

87. ?al ?ijamatikü Pen tukxü lugar?unakü

$$
\begin{aligned}
& \text { Pal } 2 \mathrm{i}=\text { jamat-i }=\mathrm{k} \quad \text { Ren tuk=xü lugar-Punak } \\
& \text { al } \mathrm{A} 3(\mathrm{ABS})=\text { llegar- } \mathrm{COMD}=\mathrm{AN} \text { en uno }=\mathrm{EV} \text { lugar-DIM }
\end{aligned}
$$

\begin{tabular}{|c|c|}
\hline 38. jemxü?k ?ijo:yi & 89. jemxü?k ?ijo:yi \\
\hline $\mathrm{jem}=\mathrm{x} \ddot{\mathrm{u}}=\mathrm{k} \quad \mathrm{i} i=\mathrm{jo}: \mathrm{y}-\mathrm{i}$ & jem $=x \ddot{u}=k \quad$ Pi $=j o: y-i$ \\
\hline $\mathrm{A} 3(\mathrm{ABS})=$ perder $-\mathrm{COMD}$ & $\mathrm{A} 3(\mathrm{ABS})=$ perder $-\mathrm{COMD}$ \\
\hline
\end{tabular}

87. En cuanto llegó en un lugarcito,

90. je? ?u:ra ?ijamati jem je? jayka?k

$$
\begin{aligned}
& \text { je? 2u:ra ?i=jamat-i jem je? jaykak } \\
& \text { ese hora } \mathrm{A} 3(\mathrm{ABS})=\text { llegar-COMD alli ese gente }
\end{aligned}
$$

90. A esa bora llegó abí la gente,

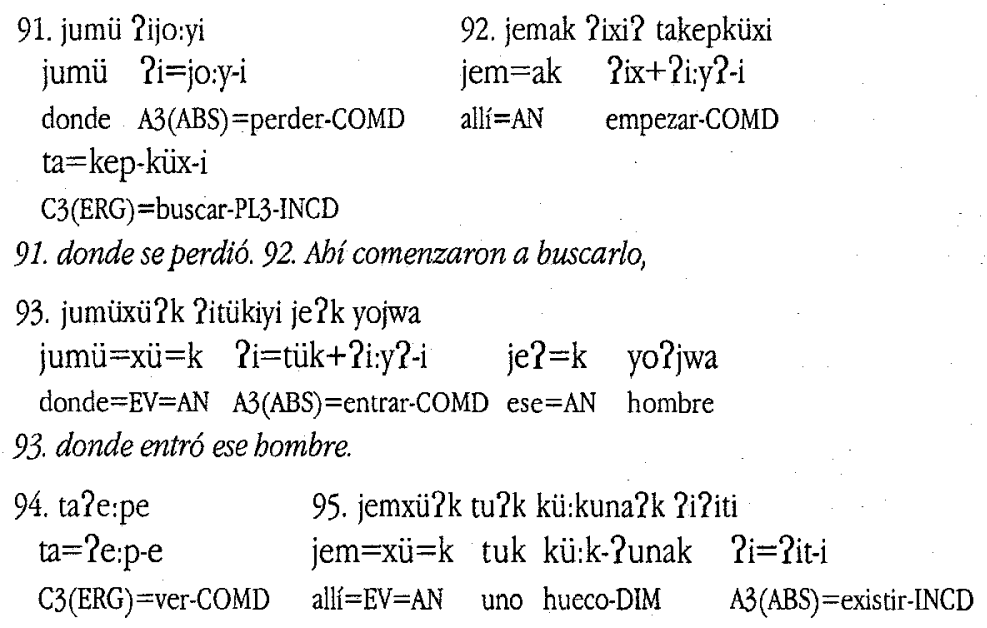

94. Cuando ven 95. que abi estaba dizque un buequito.

96. mü:xü?k ?inünküxi

mü: $t=x \ddot{u}=k \quad$ i $=$ nüm-küx-i

$\mathrm{y}=\mathrm{EV}=\mathrm{AN} \quad \mathrm{A} 3(\mathrm{ABS})=\mathrm{decir}-\mathrm{PL} 3-\mathrm{INCD}$

96. Y dizque ellos estaban diciendo: 


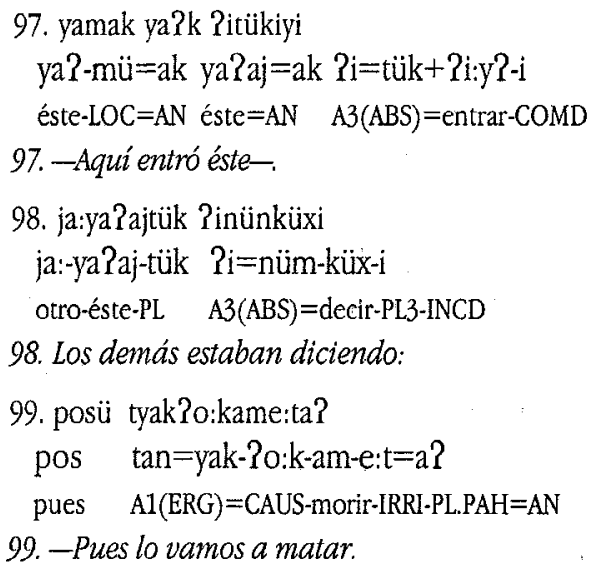

100. tyak?o:kame:ta? ya?aj

$\begin{array}{ll}\tan =y a k-P o: k-a m-e: t=a ? & \text { ya?aj } \\ \mathrm{A} 1(\mathrm{ERG})=\mathrm{CAUS} \text {-morir-IRRI-PL.PAH=AN } & \text { éste } \\ \text { 100. Vamos a matar a éste- } & \end{array}$

101. je? ?u:ra ?ixi?k tyaknaxküxi küpi

$$
\begin{aligned}
& \text { je? ?u:ra 2ix+?i:y?-i=k ta=yak-nax-küx-i küpi } \\
& \text { ese hora empezar-COMD }=A N \quad C 3(E R G)=C A U S-p a s a r-P L 3-I N C D \text { leña }
\end{aligned}
$$

101. A esa bora comenzaron a acarrear leña.
102. ta?naxü küpi $\quad 103$. komo tujtujko tare:a püne? ta?na=xü küpi komo tujtujko tare:a pün=je? mucho $=E V$ leña como seis tarea probalemente=ese

102. Era bastante leña. 103. Creo que eran como seis tareas.

104. ta?na seme tatu:tküxayik küpi ta?na seme ta=tu:t?-küx-ay-i=k küpi mucho muy $\mathrm{C} 3(\mathrm{ERG})=$ poner-PL3-APL2-COMD $=\mathrm{AN}$ leña 104. Le echaron mucha leña.

105. dejem tyakjüntzi:küxayixü ja? je?xü küpi de-jem ta=yak-jün-tzi:y?-küx-ay-i=xü ja? de-allí $\quad$ C3(ERG) $=$ CAUS-fuego-agarrar-PL3-APL2-COMD $=E V$ él je?=xü küpi ese $=E V \quad$ leña 105. De abi le prendieron esa leña. 
106. jemxü Pitoyek jamaxü küpi jem $=x \ddot{u} \quad \mathrm{i}=$ toy-e $=\mathrm{k} \quad$ jamaj $=\mathrm{xü}$ küpi alli $=\mathrm{EV} \quad \mathrm{A} 3(\mathrm{ABS})=$ quemarse $\cdot \mathrm{INCD}=\mathrm{AN}$ aquel $=\mathrm{EV} \quad$ leña 106. Abi dizque se estaba quemando esa leña.

107. ?itoy 108. ?itoyi

$\mathrm{i} i=$ toy- $\mathrm{i}$ $\mathrm{i} \mathrm{i}=$ toy- $\mathrm{i}$ $\mathrm{A} 3(\mathrm{ABS})=$ quemarse $-\mathrm{INCD} \quad \mathrm{A} 3(\mathrm{ABS})=$ quemarse $-\mathrm{INCD}$ 107. Se estaba quemando. 108. Se estaba quemando.

109. jemxü Pitoyika?i kü:kpi je?k ko?yaj jem=xü $\mathrm{i} i=$ toy-i-ka?-i küik-pi je?=k allí=EV $\mathrm{A} 3(\mathrm{ABS})=$ quemarse-NMZR-TERM-COMDhueco-LOC $\quad$ ese $=\mathrm{AN}$ ko?yaj diablo

109. Dizque allá en el bueco se acabó de quemar ese diablo.

110. despue?s tatunküxnok ?amü despues $\mathrm{ta}=$ tun-küx-nü- $\mathrm{i}=\mathrm{k} \quad$ Pam después $\quad \mathrm{C} 3$ (ERG) $=$ hacer-PL3-ya-INCD $=\mathrm{AN}$ huapango 110. Después ya bacian buapango.

111. ka:ja jamatu ja? $\mathrm{ka}:=\mathrm{ja}:=$ jamat-u $\quad$ ja? $\mathrm{NEG}=\mathrm{MIRAT}=$ llegar-COMI él 111. Ya no llegó él (el diablo),

112. porke je?k ?o:kikak ?i?itno porke je?=k $\quad$ o: $k$-ik $=\mathrm{ak} \quad$ ?i=?it-nü-e porque ese $=\mathrm{AN}$ morir $-\mathrm{PCP}=\mathrm{AN} \quad \mathrm{A} 3(\mathrm{ABS})=$ existir-ya- $\mathrm{INCD}$ 112. porque él ya estaba muerto.

113. ka:ja jamatu ja? $\mathrm{ka}:=\mathrm{ja}:=\mathrm{jamat}-\mathrm{u} \quad$ ja? $\mathrm{NEG}=\mathrm{MIRAT}=$ llegar-COMI él 113. Ya no llegó él. 
1. Pituk tukü kumpa:ne mü:tak je? ja:tukaj ?ikumpa:ne

?it-u=k tuk kumpa:ne mü:t=ak je? ja:-tuk-?aj

existir-COMI $=\mathrm{AN}$ uno compadre con $=\mathrm{AN}$ ese otro-uno-RLTVZR

?i=kumpa:ne

$\mathrm{A} 3(\mathrm{POSD})=$ compadre

1. Hubo un compadre con el otro su compadre.

2. tomekak tachikxi

3. nümpa ja?

tomek $=a k$ ta $=$ chikx-i nüm-paja?

cerca $=A N \quad$ C3 $(\mathrm{ERG})=$ tener_bajo_cuidado-INCD decir-INCIII él

2. (El primer compadre) tiene cerca (de su casa a su compadre). 3. Dice él.

4. tomekak tachikxi

tomek $=\mathrm{ak}$ ta $=$ chikx-i

cerca $=\mathrm{AN} \quad \mathrm{C} 3(\mathrm{ERG})=$ tener_bajo_cuidado-INCD

4. Lo tiene cerca (de su casa a su compadre).

5. mü: ?e:püpa? 6. Re:püp teja?

mü:t Pe:p-ü-pa $=a$ ? $\quad$ ?e:p-ü-pa te?ej=a?

y ver-INV-INCI.I $=\mathrm{AN}$ ver-INV-INCI.I ahora $=\mathrm{AN}$

5. Y (el compadre que vive cerca) lo está vigilando. 6. Lo está vigilando abora.

7. mü:tü ja? ?inükxikü takü?xtekepe ?ikumpa:ne

mü:t ja? $2 \mathrm{i}=$ nükx-i=k $\quad$ ta $=$ kü? $x+$ ta-kep-e

$y \quad$ él $\quad A 3(A B S)=i r-C O M D=A N \quad C 3(E R G)=$ pie-buscar- $-N C D$

?i=kumpa:ne

$\mathrm{A} 3(\mathrm{POSD})=$ compadre

7. Yél (el que vigila) se fue a preguntarle a su compadre (al que vive cerca).

8. nümpakü

nüm-pa $=k$

decir-INCLII $=\mathrm{AN}$

8. Dice:

9. kumpa:ne ti: mi:s mi ka: yoxtumpa

kumpa:ne ti:mi:tz mi=ka:=yox-e-tun-pa

compadre qué tú $\mathrm{B} 2(\mathrm{ABS})=\mathrm{NEG}=$ trabajar-NMZR-hacer-INCI.I

9. -Compadre, ique tú no trabajas?- 
10. tü:ya?mpikoj mi?iti

$$
\begin{array}{ll}
\begin{array}{l}
\text { tüi:y-an-pi }=\text { koj } \\
\text { colgar-NMZR_INSTR-LOC=nomás }
\end{array} & \min =\text { A2 }(\mathrm{ABS})=\text { existir-INCD }
\end{array}
$$

10. - Nomás estás en la bamaca-

11. ?ixoke

Pi $=$ xok-e

$\mathrm{A} 3(\mathrm{ABS})=$ acostarse $\cdot \mathrm{INCD}$

11. Él (el compadre que es observado) estaba acostado.

12. ?ü:sü tayoxetuni

Pü:tz $\tan =$ yox-e-tun-i

yo $\quad \mathrm{A} 1(\mathrm{ABS})=$ trabajar-NMZR-hacer-INCD

12. -Yo trabajo.

13. tayoxetuni kuyjotpi

$\tan =$ yox-e-tun-i kuy-jot-pi

$\mathrm{A} 1(\mathrm{ABS})=$ trabajar-NMZR-hacer-INCD palo-entraña-LOC

13. Yo trabajo en el monte.

14. taxtojka?tzi? kuyjotii

$$
\operatorname{tax}=\text { toj-ka?tz-Pi:y?-i }
$$$$
\text { kuy-jot }
$$

$\mathrm{Cl}(\mathrm{ERG})=\mathrm{APL}$ - - cortar-DIR:adentro-INCD palo-entraña

14. Macheteo en el monte.

15. tayu?ji yampa?k tzu?kxü?k 16. jajüp

$$
\tan =y u: j 2-i
$$
yampa?=

$\mathrm{A} 1(\mathrm{ABS})=$ rozar- $\mathrm{INCD}$ tanto $=\mathrm{AN}$

tzu?kx-ük

jaj-ü-pa

15. Rozo y está todo abuatozo. (16) Hace calor,

17. ?u:ji jotpi, tantojwintojwitij xujku?s

$$
\begin{aligned}
& \text { Pu:ji jot-pi tan=toj-wintoj-wit-i-j xujkutz } \\
& \text { garrapataentraña-LOC } \mathrm{Al}(\mathrm{ABS})=\mathrm{API} 4 \text {-cara-andar-INCD-INVD.I talzahuate } \\
& \text { 17. además bay garrapatas adentro (del monte), me caminan en la cara los }
\end{aligned}
$$

18. mü:t mi:sï yankoj x?e:pe

$$
\begin{array}{lll}
\text { mü:t mi:tz ya?-mü=koj tax=?e:p-e } & \\
\text { y tú éste-LOC=nomás } & \text { C1(LOCAL)=ver-INCD } \\
\text { 18. Mientras aqui yo nomás te veo a tí } & \\
\begin{array}{lll}
\text { 19. mi?iti tü:ya?mpi } & \text { tü:y-an-pi } & \text { minnoxoke } \\
\text { min=?it-i } & \text { colgar-NMZR INSTR-LOC } & \mathrm{A} 2(\mathrm{ABS})=\text { acostarse-INCD }
\end{array}
\end{array}
$$


19. que estás aquí en la bamaca 20. acostado.

$\begin{array}{lll}\text { 21. jumej mixtuni kumpa:ne } & \text { 22. ka: jumej ja?jü } \\ \text { jumej mix=tun-i } & \text { kumpa:ne } \quad \begin{array}{l}\text { ka:=jumej jaj } \\ \text { cómo C2(ERG)=hacer-INCD compadre } \quad \text { NEG=cómo ese }\end{array} \\ \text { 21. ¿Cómo le baces compadre? - 22. - No es nada eso, }\end{array}$

23. ta?itüp tu?k yoxena?k

$$
\begin{aligned}
& \text { ta }=\text { ?it-ü-pa tuk yox-e-nak } \\
& B 1(\mathrm{ABS})=\text { existir-INV-INCIII uno trabajar-NMZR-DIM }
\end{aligned}
$$

23. pues tengo un trabajito.

24. jaj tanto:kpe tü?ni

jaj $\tan =$ to:k-pe tü:n?-i

ese $\mathrm{A} 1(\mathrm{ERG})=$ vender-INCI.T cagar-NMZR

24. Vendo esa caca-.

25. mü:tak ?inümi ja:tukaj ?ikumpa:ne

$$
\begin{aligned}
& \text { mü: } t=a k \text { ?i=nüm-i ja:-tuk-2aj } \quad \text { i } i=k u m p a: n e \\
& y=A N \quad A 3(A B S)=\text { decir-INCD otro-uno-RLIVZR } \quad A 3(P O S D)=\text { compadre } \\
& \text { 25. } Y \text { dice su otro compadre: }
\end{aligned}
$$

$$
\begin{aligned}
& \text { 26. posü ?oya 27. nümpaxü ja? } \\
& \text { pos Roya nüm-pa }=x \ddot{u} \text { ja? } \\
& \text { pues bueno decir-INCI.I=EV él } \\
& \text { 26. -Pues bueno-, 27. dizque dice él. }
\end{aligned}
$$

28. jama:k ja:tukaj ?ikumpa:ne nükxwa? taküixtekepe

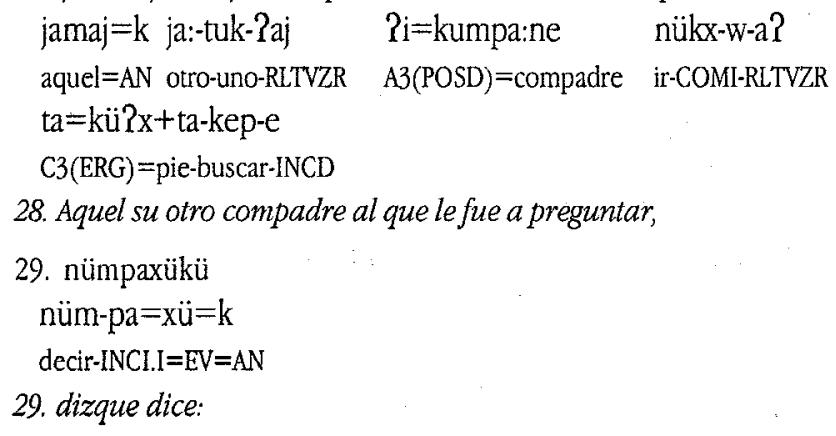

29. nümpaxükü nüm-pa $=\mathrm{xü}=\mathrm{k}$

29. dizque dice:

30. pos je? tanto:kpe kumpa:ne, tü?ni pos je? tan=to:k-pe kumpa:ne tü:n?-i

pues ese $\mathrm{A} 1(\mathrm{ERG})=$ =ender-INCI.T compadre cagar-NMZR

30. -Pues eso es lo que vendo compadre, caca-. 
31. Roya, 32. ?oya,

Poya ?oya

bueno bueno

31. - Está bien, 32. está bien-.

33. posü je? nükxam ?ipiwkote japom

pos je? nükx-am $2 \mathrm{i}=$ piw-kot-e japo:m

pues ese ir-IRRI $\quad \mathrm{A} 3(\mathrm{ABS})=$ recoger-juntar-INCD mañana

33. Pues él va a ir a recoger mañana.

34. Pawtü:spa tükxam xpiwkote

Pawtui:tzpa ta=nükx-am tax=piw-kot-e

temprano $\quad B 1(\mathrm{ABS})=$ ir-IRRI $\quad \mathrm{C} 1(\mathrm{ERG})=$ recoger-juntar-INCD

34. - Temprano voy a ir a recoger.

35. mü:t nükxa?nampok xto:ke 36. nümpaxü?k

mü:t nükx-a?n=?ampok tax=to:k-e nüm-pa=xü=k

y ir-IRRD=también $\quad \mathrm{Cl}(\mathrm{ERG})=$ vender $-\mathrm{INCD} \quad$ decir-INCIII $=\mathrm{EV}=\mathrm{AN}$

35. Y también voy a ir a vender-, 36. dizque dice él.

37. je?k kumpa:ne ?inuikxixük ?awtü:spa

je?=k kumpa:ne $2 \mathrm{i}=$ nükx-i=xü=k$\quad$ Pawtü:tzpa

ese $=\mathrm{AN}$ compadre $\quad \mathrm{A} 3(\mathrm{ERG})=\mathrm{ir}-\mathrm{COMD}=\mathrm{EV}=\mathrm{AN}$ temprano

37. Ese compadre dizque se fue temprano.

38. ?awtü:spaxük ?inükxi je? ja:tukaj kumpa:ne tapiwkote tü?ni

Pawtü:tzpa $=x \ddot{u}=k$ Pi=nükx-i je? ja:-tuk-?aj kumpa:ne temprano $=\mathrm{EV}=\mathrm{AN} \quad \mathrm{A} 3(\mathrm{ERG})=\mathrm{ir}-\mathrm{COMD}$ ese otro-uno-RLTVZR compadre ta $=$ piw-kot-e tü:n?-i

C3(ERG) $=$ recoger-juntar-INCD cagar-NMZR

38. Temprano se fue ese otro compadre a recoger caca.

39. mesko warrilxü tapiwkoti

metzko warril=xü ta=piw-kot-i

dos barril $=\mathrm{EV} \quad \mathrm{C} 3(\mathrm{ERG})=$ recoger-juntar $-\mathrm{COMD}$

39. Dizque recogió dos barriles.

40. mü:xükü ?inümi

mü:t $=x \ddot{u}=k \quad i \mathrm{i}=$ nüm- $\mathrm{i}$

$\mathrm{y}=\mathrm{EV}=\mathrm{AN} \quad \mathrm{A} 3(\mathrm{ABS})=\operatorname{decir}-\mathrm{INCD}$

40. $Y$ dizque dicen 
41. mesko warrilak tapiwkoti ?en je? japoy ?itü

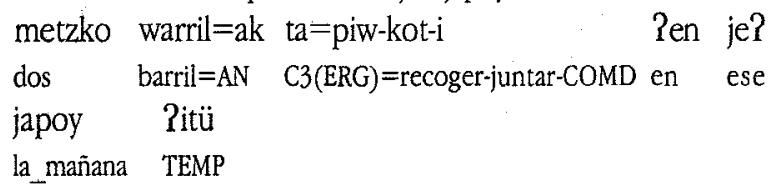

42. mü:t ja:tukaj ko:xo ?inükxixü ja? ?en je?xü ju?kupa?k tato:ke tü?ni mü:t ja:-tuk-?aj ko:xo $2 \mathrm{i}=$ nükx-i=xü ja? ?en y otro-uno-RLTVZR día $A 3(A B S)=i r-C O M D=E V$ él en je? $=x \ddot{u}$ ju?kopak ta=to:k-e tü:n?-i ese $=\mathrm{EV}$ pueblo $\mathrm{C} 3(\mathrm{ERG})=$ vender-INCD cagar-NMZR

42. Al otro día dizque se fue él, dizque en ese pueblo a vender caca

43. porke nüma?xüwa?kok ?ikumpa:ne porke nüm-a?x-ü-w-?a?=koj=k $\mathrm{i} i=k u m p a: n e$ porque decir-APL2-INV-COMI-PERF=nomás=AN A3(POSD)=compadre 43. Porque ya le babía dicho su compadre a él.

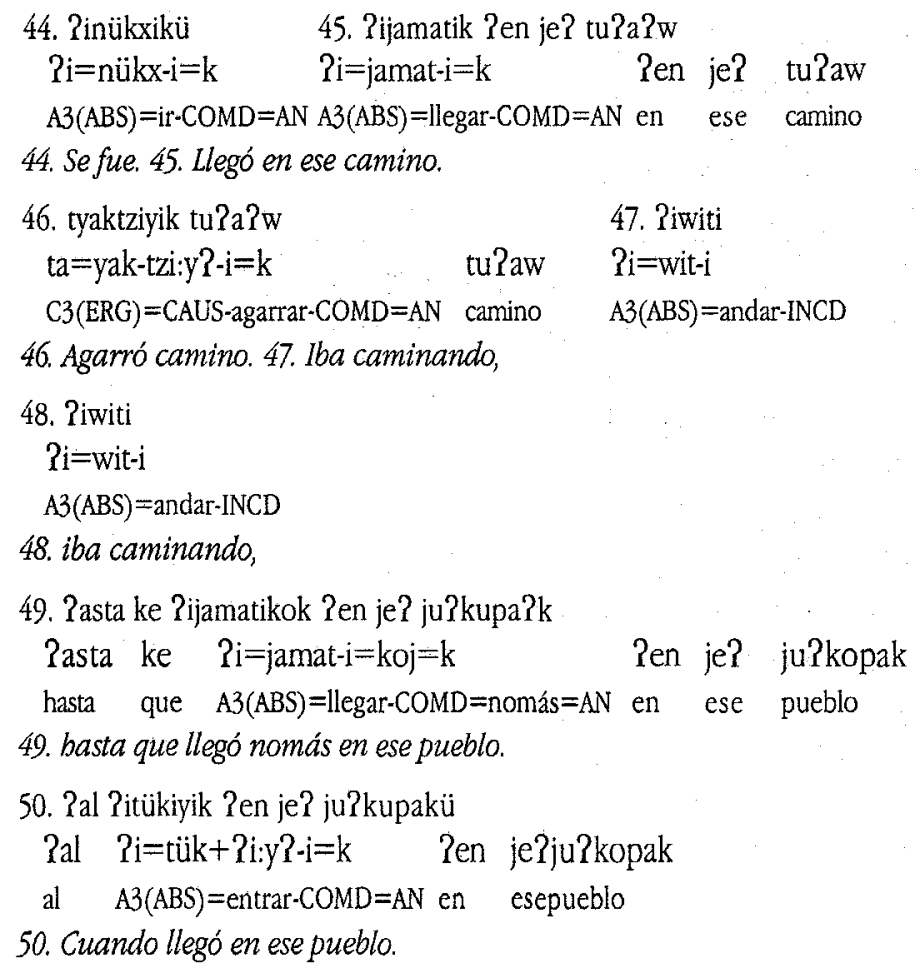


51. ?ixiyixük ?iya?xi

?ix $+2 \mathrm{i}: y \mathrm{y}-\mathrm{i}=\mathrm{xu}=\mathrm{u}=\mathrm{i} \quad \mathrm{i}=\mathrm{ya}: \mathrm{x}$ ?-i

empezar $-\mathrm{COMD}=\mathrm{EV}=\mathrm{AN} \quad \mathrm{A} 3(\mathrm{ABS})=$ gritar $-\mathrm{INCD}$

51. Dizque empezó a gritar:

52. 2iyaj tamü:minu tü?ni

Riyaj tan=mü:-mi:n?-u tü:n?-i

aquí $\mathrm{A1}(\mathrm{ERG})=\mathrm{APL} 3$-venir-COMI cagar-NMZR

52. -Aquí traje caca.

53. tanto:kpe, 54. tanto:kpe tü?ni

$\tan =$ to:k-pe tan=to:k-pe tü:n?-i

$\mathrm{A} 1(\mathrm{ERG})=$ vender-INCI.T $\mathrm{A} 1(\mathrm{ERG})=$ vender-INCI.T $\quad$ cagar-NMZR

53. Vendo, 54. vendo caca-.

55. Riya?xi 56. Riya?xixü ja?

?i=ya:x?-i $\quad$ i $i=y a: x ?-i=x u ̈ \quad$ ja?

$\mathrm{A} 3(\mathrm{ABS})=$ gritar $-\mathrm{INCD} \quad \mathrm{A} 3(\mathrm{ABS})=$ gritar-INCD $=\mathrm{EV}$ él

55. Grilaba. 56. Élgritaba.

57. 2al tamotowik jama:k jayka?k

Pal $\mathrm{ta}=$ motow $-\mathrm{i}=\mathrm{k} \quad$ jamaj $=\mathrm{k}$ jaykak

al $\quad \mathrm{C}$ (ERG)=escuchar-COMD $=\mathrm{AN}$ aquel=AN gente

57. Cuando escucbó aquella gente

58. tato:ke?aj tü?ni

ta=to:k-e-Paj tü:n?-i

C3(ERG) $=$ vender-INCD-RLTVZR cagar-NMZR

58. que él vendía caca,

59. je? ?u:raxük ?imini polisyatük

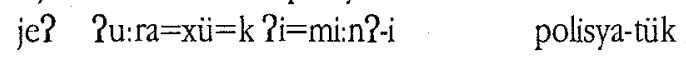

ese hora $=\mathrm{EV}=\mathrm{AN} \quad \mathrm{A} 3(\mathrm{ABS})=$ venir $-\mathrm{COMD}$ policia $\mathrm{PL}$

59. a esa bora llegaron los policías

60. ?imü:nükxküxuk wepxü?ü ju?kupaktükpi

$\mathrm{Pi}=$ mü:-nükx-küx-u=k wew-pi=xü ju?kopak-tük-pi

$\mathrm{A} 3(\mathrm{ERG})=\mathrm{APL} 3-\mathrm{ir}-\mathrm{PL} 3-\mathrm{COMI}=\mathrm{AN} \quad$ allá-LOC $=\mathrm{EV}$ pueblo-casa-LOC

60. Ellos se lo llevaron dizque allá al palacio municipal.

61. ?ijamatixü?k ?ikü?p ko?paktumpa?aj

$\mathrm{i} i=$ jamat $-\mathrm{i}=\mathrm{xü}=\mathrm{k} \quad \mathrm{i}=\mathrm{i}$ ü? $\mathrm{p} \mathrm{pi}$

$\mathrm{A} 3(\mathrm{ABS})=$ llegar $-\mathrm{COMD}=\mathrm{EV}=\mathrm{AN} \mathrm{A} 3(\mathrm{POSD})=$ mano- $\mathrm{LOC}$ 


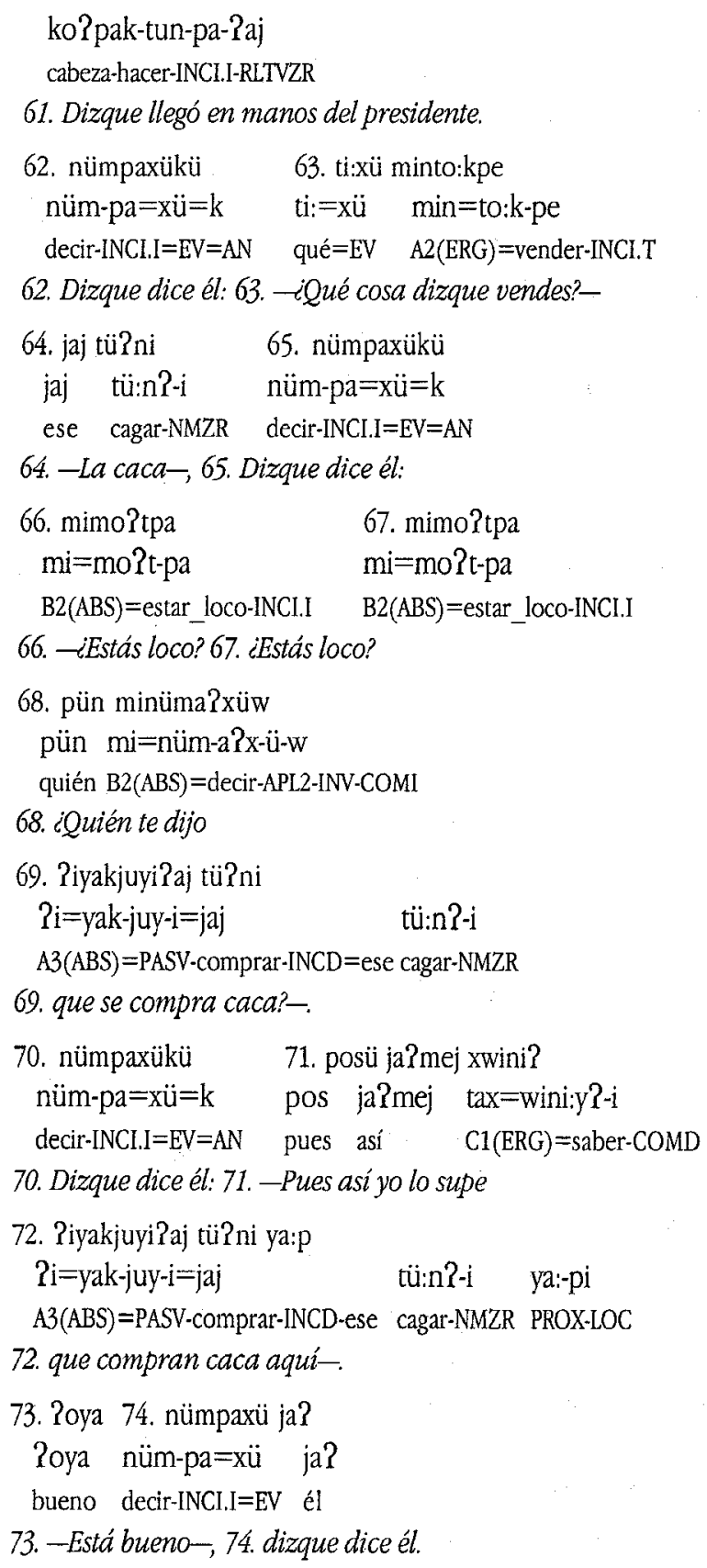

72. Piyakjuyi?aj tü?ni ya:p

?i=yak-juy-i=jaj tü:n?-i ya:-pi

73. Poya 74. nümpaxü ja?

2oya nüm-pa $=x \ddot{u} \quad$ ja?

bueno decir-INCI.I=EV él

73. -Está bueno-, 74. dizque dice él. 
75. posü je? ?u:raxüik ?iyaktze:ki

pos je? $2 \mathrm{u}: \mathrm{ra}=\mathrm{xu}=\mathrm{u}=\mathrm{k} \quad \mathrm{i}=$ yak-tze:k-i

pues ese hora $=\mathrm{EV}=\mathrm{AN} \quad \mathrm{A} 3(\mathrm{ABS})=\mathrm{PASV}$-regañar-COMD

75. Pues dizque a esa bora se le regañó,

76. nümpaxükü $\quad$ 77. Riyaktze:ki

nüm-pa $=x \ddot{u}=\mathrm{k} \quad$ ?i=yak-tze: $k-\mathrm{i}$

decir-INCLII $=\mathrm{EV}=\mathrm{AN} \quad \mathrm{A} 3(\mathrm{ABS})=\mathrm{PASV}$-regañar-COMD

76. dizque dicen. 77. Lo regañaron

78. ?itze:kiyi?akü ko?paktumpa? de je? ju?kupaktükü ja?k ko?paktumpa?aj

$$
\text { Pi=tze:k-Pi:y?-i-y=ak ko?pak-tun-pa-? }
$$

$\mathrm{A} 3(\mathrm{ABS})=$ regañar-DIR:adentro-COMD-INVD. $\mathrm{C}=\mathrm{AN}$ cabeza-hacer-INCLI.NMZR

de je? ju?kopak-tük ja?=k ko?pak-tun-pa-?aj

de ese pueblo-casa ćl=AN cabeza-hacer-INCII-RLTVZR

78. Lo regañó el jefe de ese palacio, el presidente.

79. nümpaxükü $\quad 80$. posü ?oyamento nükxü 81 nükxü

nüm-pa $=\mathrm{xü}=\mathrm{k}$ pos ?oyamento nükx-ü nükx-ü

decir-INCI. $=\mathrm{EV}=\mathrm{AN}$ pues ahorita ir-IMPR ir-IMPR

79. Dizque dice: 80. -iPues vete aborital-81. -1Vete

82. jumü mimini $\quad$ 83, mü:t ka:mija:mi?n

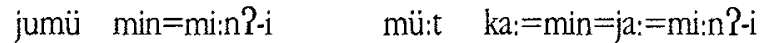

donde $\mathrm{A} 2(\mathrm{ABS})=$ venir-COMD y $\quad \mathrm{NEG}=\mathrm{A} 2(\mathrm{ABS})=\mathrm{MIRAT}=$ venir-INCD

82. bacia el lugar de donde tú veniste 83. y ya no vengas!-

84. ka:mija:mi?n $\quad$ 85. nümpaxü ja?

$\mathrm{ka}:=\min =\mathrm{ja}:=\min 2-\mathrm{i} \quad$ nüm-pa=xü ja?

$\mathrm{NEG}=\mathrm{A} 2(\mathrm{ABS})=\mathrm{MIRAT}=$ venir-INCD decirIINCI.I $=\mathrm{EV}$ él

84. -iYa no vengas!-, 85. dizque dice él.

86. je? ?u:raxükü Piyü?ki

je? $2 u: r a=x u ̈=k \quad$ Pi=yü?k-i

ese hora $=\mathrm{EV}=\mathrm{AN} \quad \mathrm{A} 3(\mathrm{ABS})=$ salir $-\mathrm{COMD}$

86. Dizque a esa bora se salió (del pueblo).

87. ?iyü?knexükü tu:ntu

Pi=yü?k-nü-i=xü=k tu:ntu

$\mathrm{A} 3(\mathrm{ABS})=\mathrm{salir}-\mathrm{ya}-\mathrm{COMD}=\mathrm{EV}=\mathrm{AN} \quad$ triste

87. Dizque ya se salió triste. 
88. porke yaktojtze:ki:wa?koj ja?

porke yak-toj-tze:k-2i:y?-w-?a?=koj ja?

porque PASV-APL4-regañar-DIR:adentro-COMI-PERF=nomás él

88. Porque ya lo regañaron nomás (con palabras).

89. tri:ste ?ijamatik ?ixküjpu:t tu?awü

tri:ste $2 \mathrm{i}=$ jamat-i=k $\quad$ Pixküj+pu:t tu?aw

triste $\mathrm{A} 3(\mathrm{ABS})=\mathrm{ll}$ egar-COMD $=\mathrm{AN}$ mitad camino

89. Llegó triste hacia la mitad del camino

90. 2ipitzümixük ko?yaj 91. nümpaxükü

$\mathrm{Pi}=$ pitzüm- $\mathrm{i}=\mathrm{xü}=\mathrm{k} \quad$ ko?yaj $\quad$ nüm-pa $=\mathrm{x} \ddot{u}=\mathrm{k}$

$\mathrm{A} 3(\mathrm{ABS})=$ salir-COMD $=\mathrm{EV}=\mathrm{AN}$ diablo decir-INCII $\mathrm{I}=\mathrm{EV}=\mathrm{AN}$

90. de donde dizque salió el diablo. 91. Dizque dice:

92. ti: mijatüp 93. nümpaxü?k ko?yaj

ti: mi=jat-ü-pa nüm-pa $=x u ̈=k \quad$ ko?yaj

qué $\quad B 2(A B S)=$ suceder-INV-INCI.I decir-INCII $I=E V=A N$ diablo

92. -Qué cosa tienes? - 93. Dizque dice el diablo.
94. yaj tajatüw 95. nümpaxü ja? ya?aj ta=jat-ü-w nüm-pa=xü ja?
éste $\quad B 1(\mathrm{ABS})=$ suceder-INV-COMI decir-INCI.I=EV él
94. -Esto es lo que me pasó- 95. Dizque dice él.

96. ?oya 97. nümpaxü ja? 98. pero mimü:nükxam tumi?n, ?oya nüm-pa $=x \ddot{u}$ ja? pero min=mü:-nükx-am tumin bueno decir-INCI.I=EV él pero $\mathrm{A2}(\mathrm{ERG})=\mathrm{APL} 3$-rir-IRRI dinero

96. -Está bien-. 97. Dizque dice él. 98. -Pero vas a llevarte dinero.

99. mimü:nükxam me:nyu $\quad 100$. jemü Pi?iti me:nyu min=mü:-nükx-am me:nyu jem ?i=?it-i me:nyu $\mathrm{A} 2(\mathrm{ERG})=\mathrm{API} 3$-ir-IRRI dinero allí $\mathrm{A}$ (ABS)=existir-INCD dinero 99. Vas a llevarte dinero. 100. Abi está el dinero.

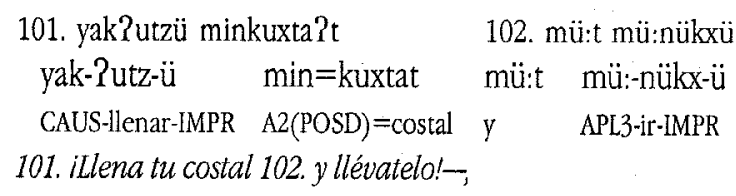

103. nümpaxü?k je? ko?yaj, nüm-pa $=$ xü $=k \quad j e ?$ ko?yaj decir-INCI. $=\mathrm{EV}=\mathrm{AN}$ ese diablo 103. dizque dice el diablo. 
104. tyak?utzixük maktaxko kuxtatü

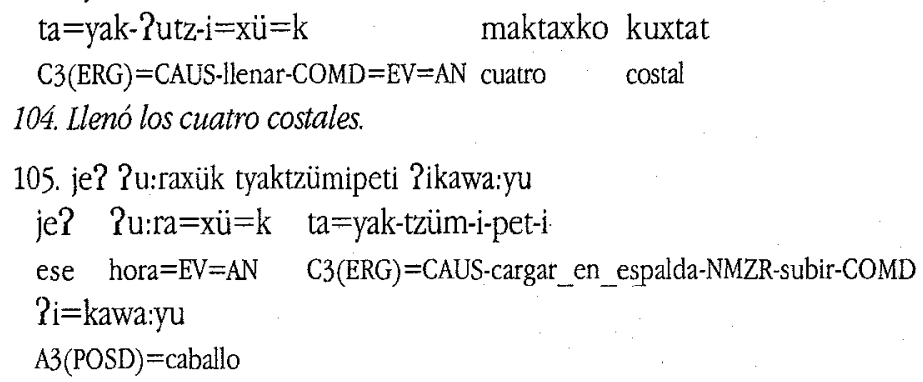

113. tamü:kapxe ?ikawa:yu

$$
\begin{array}{ll}
\mathrm{ta}=\mathrm{mü}:-\mathrm{kapx}-\mathrm{e} & \mathrm{P} \mathrm{i}=\mathrm{kawa}: \mathrm{yu} \\
\mathrm{C} 3(\mathrm{ERG})=\mathrm{APL} \text {-hablar-INCD } & \mathrm{A} 3(\mathrm{POSD})=\text { caballo }
\end{array}
$$


113. Le bablaba a su caballo.
114. tanaswopi?
115. Pikapxe tüwi?k
ta $=$ natz-wop-i: $y 2-i$
?i=kapx-e
tüw-ik
$\mathrm{C} 3$ (ERG) =atrás-golpear-DIR:adentro-INCD A3(ABS)=hablar-INCD apretar-PCP
114. Lo arreaba (a su caballo). 115. Hablaba recio

116. jumük Pi?iti ?ikumpa:ne

$$
\begin{array}{lll}
\text { jumü=k } & \mathrm{P}=\text { = } \mathrm{it}-\mathrm{i} & \mathrm{i} \mathrm{i}=\text { kumpa:ne } \\
\text { donde }=\mathrm{AN} & \mathrm{A} 3(\mathrm{ABS})=\text { existir-INCD } & \mathrm{A} 3(\mathrm{POSD})=\text { compadre }
\end{array}
$$

116. abi donde está su compadre,

117. tome?k de ?itükü

tomek de $\mathrm{i} \mathrm{i}=$ tük

cerca de $\mathrm{A} 3(\mathrm{POSD})=$ casa

117. cerca de su casa.

118. mü: jemxük ?ikumpa:ne ?i?iti, Pitükpi

mü:t jem=xü=k $\quad$ i $=$ kumpa:ne $\quad$ i $=$ ?it-i

y alli $=\mathrm{EV}=\mathrm{AN} \quad \mathrm{A} 3(\mathrm{POSD})=$ compadre $\quad \mathrm{A} 3(\mathrm{ABS})=$ existir $-\mathrm{N} \mathrm{NCD}$

ii =tïk-pi

$\mathrm{A} 3(\mathrm{POSD})=$ casa-LOC

118. Y abi estaba su compadre, estaba en su casa.

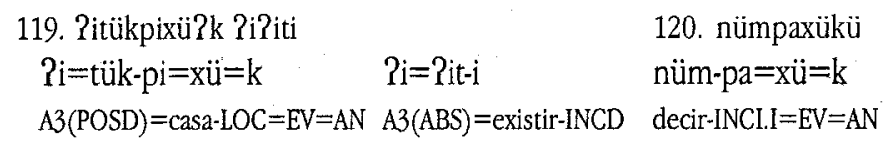

119. En su casa estaba. 120. Dizque dice él:

121. ?u:ta ya?twa? kumpa:ne

Ru:ta ya?t-w-?a? kumpa:ne

EXCLAM llegar_aquí-COMI-PERF compadre

121. -iUb!, ya llegó el compadre-,

122. nümpaxï ja? 123. je? ?u:raxü?k ta?e:pi

nüm-pa=xü ja? je? ?u:ra=xü=k ta=?e:p-i

decir-INCI.I $=\mathrm{EV}$ él ese hora $=\mathrm{EV}=\mathrm{AN} \quad \mathrm{C3}(\mathrm{ERG})=$ ver-COMD

122. dizque dice él. 123. Dizque a esa bora lo vió

124. mesko kuxtatxü me:nyo tamü:mi?n

metzko kuxtat=xü me:nyo ta=mü:-mi:n?-i

dos costal $=\mathrm{EV}$ dinero $\quad \mathrm{C} 3(\mathrm{ERG})=\mathrm{APL} 3$-venir $-\mathrm{N} \mathrm{NCD}$

124. que traía dos costales de dinero. 
125. mü:xük tanümayi ?imajaw

$$
\begin{array}{lll}
\text { mü: } t=x \ddot{u}=k & t a=n u ̈ m-a y-i & 2 \mathrm{i}=\text { majaw } \\
y=E V=A N & C 3(E R G)=\text { decir-APL2-COMD } & A 3(\text { POSD)=mujer }
\end{array}
$$

\section{Yle dijo a su mujer.}

126. me:jitwa? kumpa:ne

$$
\begin{aligned}
& \text { me:j?-Pit-w-Pa? kumpa:ne } \\
& \text { engañar-PAH-COMI-PERF compadre } \\
& \text { 126. -Ya nos cbingó el compadre }
\end{aligned}
$$

127. porke ta?nak me:nyu tamü:mi?n porke ta?na=k me:nyu ta=mü:-mi:n?-i

porque mucho $=\mathrm{AN}$ dinero $\mathrm{C} 3(\mathrm{ERG})=\mathrm{APL} 3$-venir-INCD

127. porque trae bastante dinero-,

128. nümpaxü ja?

nüm-pa $=x \ddot{i} \quad j a ?$

decir-INCI.I=EV él

128. dizque dice él.

129. ja:tukaj ko:xo ja:je?xük ?inükxi

$$
\text { ja:-tuk-?aj ko:xoja:-je?=xü=k } \quad 2 \mathrm{i}=\text { nükx-i }
$$

otro-uno-RLTVZR día otro-ese $=\mathrm{EV}=\mathrm{AN} \mathrm{A}$ ( $(\mathrm{ABS})=\mathrm{ir}-\mathrm{COMD}$

129. Al otro dia dizque se fue el otro (el compadre sin dinero..

130. kumpa:ne 131. ti: kumpa:ne 132. naxa

kumpa:ne ti: kumpa:ne nax-a

compadre qué compadre pasar-IMPR

130. -iCompadre!-131. -iQué compadre?-132. -iPasal-

133. nümpaxü ja? 134. jumej mi?oyi je:p

$$
\text { nüm-pa=xü ja? jumej min=?oy-i je:-pi }
$$

decir-INCIII=EV él cómo A2(ABS)=ir_(COM)-COMD DIST-LOC

133. Dizque dice él: 134 . -iCómo estuvo donde fuiste?-

135. ?oyu sye:mpre xto:ke tü?ni

$$
\begin{aligned}
& \text { Poy-u sye:mpre tax=to:k-e tü:n?-i } \\
& \text { ir_(COM)-COMI siempre } \quad \text { C1(ERG)=vender-INCD cagar-NMZR } \\
& \text { 135. -Fui siempre a vender caca-, }
\end{aligned}
$$

136. nümpaxü ja? 137. pe:ro yakjuypa seme

nüm-pa=xü ja? pe:ro yak-juy-pa seme

decir-INCIII $=\mathrm{EV}$ él pero PASV-comprar-INCI.I muy

136. dizque dice él. 137. -Pero compran mucba-, 


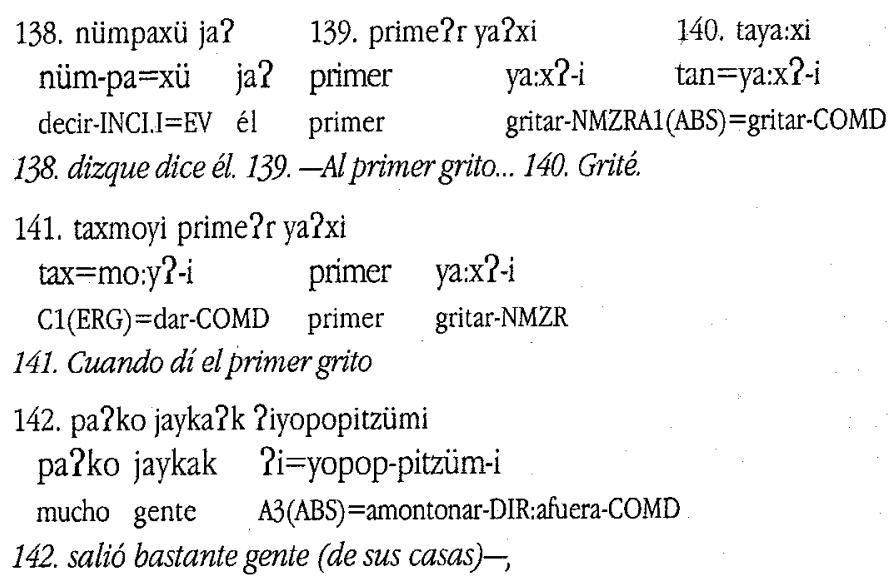

146. pos ka: naspa:tiyuk je? mesko warrilaj tü?ni

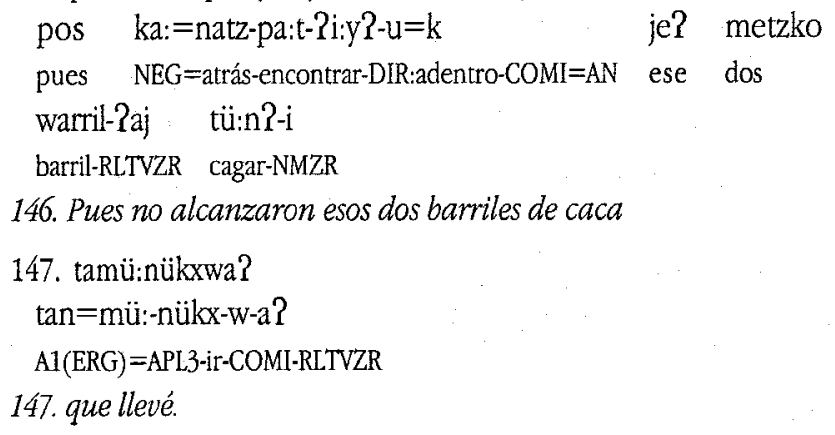

148. porke mü:t tanka:yaku? ti:k ta?na porke mü:t $\tan =\mathrm{ka}:=y a k-\mathrm{u}-\mathrm{P} \quad \mathrm{ti}:=\mathrm{k}$ ta?na porque y $\mathrm{Al}(\mathrm{ERG})=\mathrm{NEG}=\mathrm{dar}-\mathrm{COMI}-\mathrm{NMZR}$ qué $=\mathrm{AN}$ mucho 148. Y eso que no di mucbo. 
149. küjtu?k kucha:rawokoj por tu?k pe:xu küj-tuk kucha:ra-wok=koj por tuk pe:xu cada-uno cuchara-DIM=nomás por uno peso

149. De una cucbaradita por un peso.

150. küjtu?k kucha:rawokoj por tu?k pe:xu küj-tuk kucha:ra-wok=koj por tuk pe:xu cada-uno cuchara-DIM=nomás por uno peso 150. De una cucbaradita por un peso.

151. mü:t ka:naspa:tiyu

$$
\begin{array}{ll}
\text { mü:t } & \mathrm{ka}:=\text { natz-pa:t-Pi:y?-u } \\
\text { y } & \mathrm{NEG}=\text { atrás-encontrar-DIR:adentro-COMI }
\end{array}
$$

151. Y no alcanzó.

152. mü:t tijunaj pa?ko jayka?k

$\begin{array}{ll}\text { mü:t tij-u=na } & \text { pa?ko jaykak } \\ \text { y quedar-ComI=todavía } & \text { mucho gente } \\ \text { 152. Y quedó todavía mucha gente }\end{array}$

153. pün ?iwanu?a?

pün $2 \mathrm{i}=$ wa:n?-u-?a?

quién $\quad \mathrm{A} 3(\mathrm{ERG})=$ querer-COMI-RLTVZR

153. que queria-.

154. je?kok Pimotowuk ?ikumpa:ne

$$
\text { je? }=\text { koj }=\mathrm{k} \quad \mathrm{i}=\text { motow- } \mathrm{u}=\mathrm{k} \quad \mathrm{i}=\text { kumpa:ne }
$$

ese $=$ nomás $=\mathrm{AN} A 3(\mathrm{ERG})=$ escuchar $-\mathrm{COMI}=\mathrm{AN} \quad \mathrm{A} 3(\mathrm{POSD})=$ compadre

154. Eso nomás es lo que oyó su compadre.

155. ja:je?xük ?inükxi

$$
\begin{array}{ll}
\text { ja: }-j e ?=x \ddot{u}=k & 2 i=n \ddot{k} x-\mathrm{i} \\
\text { otro-ese }=E V=A N & A 3(A B S)=i r-C O M D
\end{array}
$$

155. El otro (el compadre sin dinero) dizque se fue

156. ja:tukaj ko:xo ?awtü:spa japoy ?itu

$$
\begin{array}{clll}
\text { ja:-tuk-?aj ko:xo?awtü:tzpa japoy } & \text { Ritü } \\
\text { otro-uno-RLTVZR día temprano la_mañana } & \text { TEMP } \\
\text { 156. al otro día tempranoporla mañana. } &
\end{array}
$$

157. nümpaxükü 158. ja? ใü:?tz tamü:nükxam

nüm-pa $=x \ddot{u}=k \quad$ ja? ?ü:tz $\tan =m u ̈$ :-nükx-am

decir-INCIII $=E V=A N \quad$ MIRAT $\quad$ yo $\quad \mathrm{Al}(\mathrm{ERG})=A P L 3-i r-I R R I$

157. Dizque dice él: 158. -Yo voy a llevarla (caca al otro pueblo)-, 
159. nümpaxü ja? nüm-pa $=x \ddot{u} \quad j a ?$ decir-INCI.I=EV él

159. dizque dice él.

160. tanümayixükü ?imajaw

$$
\begin{aligned}
& \mathrm{t} a=\text { nüm-ay }-\mathrm{i}=\mathrm{xu}=\mathrm{k} \quad \mathrm{i}=\text { majaw } \\
& \mathrm{C} 3(\mathrm{ERG})=\text { decir- } \mathrm{APL} 2 \mathrm{-} \mathrm{NCD}=\mathrm{EV}=\mathrm{AN} \quad \mathrm{A} 3(\mathrm{POSD})=\text { mujer } \\
& \text { 160. Le dijo a su mujer: }
\end{aligned}
$$

161. porke yakjuypa seme porke yak-juy-pa 162. nümpaxü ja? porque PASV-comprar-INCI.I muy decir-INCIII=EV él

\begin{tabular}{|c|c|}
\hline $\mathrm{P} i=$ jamat $-\mathrm{i}=\mathrm{x} \ddot{\mathrm{u}}=\mathrm{k}$ & ?en \\
\hline $\begin{array}{l}\mathrm{A} 3(\mathrm{ABS})=\text { legar } \mathrm{COMD}=\mathrm{EV}=\mathrm{AN} \\
\mathrm{ta}=\text { piw-kot-e }\end{array}$ & en ese \\
\hline $\mathrm{C3}$ (ERG) = recoger-juntar-INCD & cagar-NMZR \\
\hline
\end{tabular}
161. -Porque compran mucbo-, 162. dizque dice él.

163. ?ijamatixük ?en je? tapiwkote tü?ni

164. ja:tukaj ko:xo Pinükxixü?k tato:ke

$$
\begin{aligned}
& \text { ja:-tuk-?aj ko:xo } \quad \mathrm{i}=\text { nükx-i }=\mathrm{x} \ddot{\mathrm{u}}=\mathrm{k} \quad \text { ta=to:k-e } \\
& \text { otro-uno-RLTVZR día } \mathrm{A}(\mathrm{ABS})=\mathrm{ir}-\mathrm{COMD}=\mathrm{EV}=\mathrm{AN} \mathrm{C} 3(\mathrm{ERG})=\text { vender-1NCD } \\
& \text { 164. Al otro día se fue a venderla. }
\end{aligned}
$$

165. je? ?u:raxükü ?al Pitükiyik ju?kupakmü

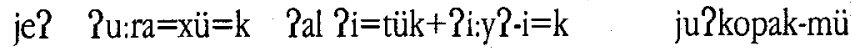
ese hora $=\mathrm{EV}=\mathrm{AN}$ al $\mathrm{A} 3(\mathrm{ABS})=$ entrar $-\mathrm{COMD}=\mathrm{AN}$ pueblo-LOC 165. Dizque a esa bora cuando entró al pueblo

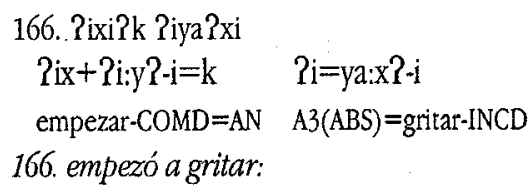

167. tanto:kpe tü?ni $\tan =$ to:k-pe tü:n?-i 168. tanto:kpe tü?ni $\mathrm{A} 1(\mathrm{ERG})=$ vender-INCI.T cagar-NMZR $\quad \mathrm{A} 1(\mathrm{ERG})=$ vender-INCI.T $\quad$ cagar-NMZR 167. -iVendo caca! 168. iVendo caca!- 
169. je? ?u:rakü tamotowküxpowikoj je?k ja:yajtük polisya

$$
\begin{aligned}
& \text { je? } P \mathrm{u}: \mathrm{ra}=\mathrm{k} \quad \mathrm{ta}=\text { motow-küx-pow- } \mathrm{i}=\mathrm{koj} \quad \mathrm{je} \mathrm{l}=\mathrm{k} \\
& \text { ese hora=AN } \mathrm{C}(\mathrm{ERG})=\text { escuchar-PL3-REPET-COMD=nomás ese=AN } \\
& \text { ja:=ya?aj-tük polisya } \\
& \text { otro=este-PL policía } \\
& \text { 169. A esa bora escucharon de vuelta los otros policías. }
\end{aligned}
$$
170. nümpaxükü 171. mimpowa?kok nüm-pa $=x \ddot{u}=k \quad$ mi:n?-pow-w-Pa? $=k o j=k$
172. je?k ?ito:kpe tü?ni 173. nümpaxü ja? je?=k $\mathrm{i} i=$ to:k-pe tü:n?-i nüm-pa=xü ja? ese $=\mathrm{AN} \quad \mathrm{A} 3(\mathrm{ERG})=$ vender-INCI. $\mathrm{T} \quad$ cagar-NMZR $\quad$ decir-INCI.I=EV él 172. ese que vende caca-, 173. dizque dicen.

174. mü:t tanümayuk mü:t $\tan =$ nüm-ay-u=k y $\quad \mathrm{A} 1(\mathrm{ERG})=$ decir-APL $2-\mathrm{COMI}=\mathrm{AN}$ 174. -Yle dije
175. ?ika:jami?na?na?k 176. nümpaxü ja? $\mathrm{Pi}=\mathrm{ka}:=\mathrm{ja}:=\mathrm{mi}: \mathrm{n} 2-\mathrm{a} \mathrm{n} n=\mathrm{ak} \quad$ nüm-pa=xü ja? $\mathrm{A} 3(\mathrm{ABS})=\mathrm{NEG}=\mathrm{MIRAT}=$ venir-IRRD $=\mathrm{AN}$ decir-INCI.I=EV él 175. que ya no viniera-, 176. dizque dice él.

177. te?ej pe?k nükxü pükta:t

$$
\begin{aligned}
& \text { te?ej pek nükx-ü pük-ta-:t } \\
& \text { ahora de_verdad ir-IMPR agarrar-IMPR-PL.PAH } \\
& \text { 177. -iY abora vayan a traérmelo! }
\end{aligned}
$$

178. nükxü pükta:t 179. nümpaxü ja?

$$
\begin{array}{lll}
\text { nükx-ü } & \text { pük-ta-:t } & \text { nüm-pa=xü ja? } \\
\text { ir-IMPR } & \text { agarrar-IMPR-PL.PAH } & \text { decir-INCl.I=EV él }
\end{array}
$$

178. Nayan a traérmelo!-, 179. dizque dice él.

$$
\begin{array}{ll}
\text { 180. je? ?u:ra nükxi Piyakpüki } & \text { 181. nümpaxükü } \\
\text { je? Pu:ra nükx-i } 2 \mathrm{i}=\text { =yak-pük-i } & \text { nüm-pa=xü=k } \\
\text { ese hora ir-COMDA3(ABS)=PASV-agarrar-INCD } & \text { decir-INCI.I=EV=AN } \\
\text { 180. A esa bora lo fueron a traer. 181. Dizque dice él. }
\end{array}
$$


182. taxnümayu:tü?s seme

tax=nüm-ay-u-:t-?ütz seme

$\mathrm{C1}(\mathrm{LOCAL})=$ decir-APL2-COMI-PL.PAH-EXCL muy

182, - Te dijimos demasiado

183. minka:jami?na?n mixto:ke

$\min =\mathrm{ka}:=\mathrm{ja}:=\operatorname{mi}: \mathrm{n}$-a?n $\quad \mathrm{mix}=\mathrm{to}: \mathrm{k}-\mathrm{e}$

$\mathrm{A} 2(\mathrm{ABS})=\mathrm{NEG}=\mathrm{MIRAT}=$ venir-IRRD C2(ERG) $=$ vender $-\mathrm{INCD}$

183. que no vinieras a vender-,

184. nümpaxü ja?

nüm-pa $=x \ddot{~} \quad$ ja?

decir-INCIII $=\mathrm{EV}$ él

184. dizque dice él.

185. ti: je? pün minjuya?xanüp tü?ni

ti: je? pün min=juy-a?x-an-ü-pa tü:n?-i

quée ese quién $\mathrm{A}$ 2(ABS)=comprar-APL2-IRR-INV-INCII cagar-NMZR

185. -iQuién es ese que te va a comprar caca?

186. xu:kpa 187. nümpaxü ja?

xu:k-pa nüm-pa $=x u ̈$ ja?

apestar-INCI.I decir-INCIII=EV él

186. Apesta-, 187. dizque dice él.

188. posü tej pe?k minükxam wep

pos te?ej pek mi=nükx-am wew-pi

pues ahora de_verdad $\mathrm{B} 2(\mathrm{ABS})=\mathrm{ir}-\mathrm{IRRI}$ allá-LOC

188. -Pues y abora te vas a ir de verdad para allá-,

189. nümpaxü ja? ju?kupaktükpi

nüm-pa=xü 'ja? ju?kopak-tük-pi

decir-INCIII $=\mathrm{EV}$ él pueblo-casa-LOC

189. dizque dice él, -al palacio municipal.

190. miyakapxüpak ko?paktumpa?

$\mathrm{mi}=$ yak-kapx-ü-pa $=\mathrm{k} \quad$ ko?pak-tun-pa-?

B2(ABS) $=$ CAUS-hablar-INV-INCIII=AN cabeza-hacer-INCI.I-NMZR

190. -Te llama el presidente-

191. je? ?u:ra Piyakmü:nükxi wotze

je? ?u:ra ?i=yak-mü:-nülkx-i wotz-e

ese hora A3(ABS)=PASV-APL3-ir-COMD jalar-NMZR

191. A esa hora lo llevaron a jalonazos. 
192. pu:ro wotze ?iyakmü:nükxi

pu:ro wotz-e $\mathrm{i} i=y a k-m u ̈:-n u ̈ k x-i$

puro jalar-NMZR A3(ABS)=PASV-APL3-ir-INCD

192. A puro jalonazos lo llevaban.

193. ?ijamatik jem ju?kupaktükpi

$\mathrm{P} i=$ jamat-i=k jem ju?kopak-tük-pi
$\mathrm{A} 3(\mathrm{ABS})=$ llegar-COMD $=\mathrm{AN}$ alli
pueblo-casa-LOC

193. Llegó abí al palacio, al palacio.

194. ju?kupaktükpi je:pak ?i?itno ja:tu?k ko?paktumpa?

ju?kopak-tük-pi je:-pi=ak $\quad$ ii=?it-nü-e

pueblo-casa-LOC DIST-LOC=AN A3(ABS)=existir-ya-INCD

ja:-tuk ko?pak-tun-pa-?

otro-uno cabeza-hacer-INCI.I-NMZR

194. En el palacio, alli estaba ya el otro jefe.

195. ju?kupaktükpi jamatu?a?koj ja? 196. nümpaxükü ju?kopak-tük-pi jamat-u-?a?=koj ja? nüm-pa $=x \ddot{u}=k$

pueblo-casa-LOC llegar-COMI-PERF=nomás él decir-INCI. $\mathrm{I}=\mathrm{EV}=\mathrm{AN}$

195. Al palacio ya nomás habia llegado. 196. Dizque dice:

197. taxnümayu:tü?s

tax=nüm-ay-u-:t-Pütz

$\mathrm{C} 1(\mathrm{LOCAL})=$ decir-APL2-COMI-PL.PAH-EXCL

197. - Te dijimos

198. minka:jami?na?n 199. taxnümayu

$\min =k a:=j a:=m i: n ?-a$ ?n $\quad$ tax $=$ nüm-ay-u

$\mathrm{A} 2(\mathrm{ABS})=\mathrm{NEG}=\mathrm{MIRAT}=$ venir-IRRD $\mathrm{C} 1(\mathrm{LOCAL})=$ decir-APL2-COMI

198. que ya no vinieras. 199. Te dije

200. minka:jami?na?n

$\min =k a:=j a:=\operatorname{mi}: n 2-a ? n$

$\mathrm{A} 2(\mathrm{ABS})=\mathrm{NEG}=\mathrm{MIRAT}=$ venir $-\mathrm{IRRD}$

200. que ya no vinieras.

201. porke ta miminpo:pakojü

porke ta $m i=m i: n 2-p o w-p a=k o j$

porque COND $\mathrm{B} 2(\mathrm{ABS})=$ venir-REPET-INCI.I=nomás

201. Porque si venías otra vez nomás 
202. taxyak?ixkejam

tax=yak-?üx + kej-am

$\mathrm{C} 1(\mathrm{LOCAL})=$ CAUS-encuerar-IRRI

202. te iba a encuerar

203. mü: xmo?a?n wope mintewajem

mü:t tax=mo:y?-a?n wop-e $\quad \min =$ tewa-jem

y $\quad$ C1(LOCAL) $=$ dar - IRRD $\quad$ golpear-NMZR $A 2($ ABS $)=$ nalga-LOC

203. y te iba a dar de golpes en tu nalga-,

204. nümpaxü je?kü ko?paktumpa?aj

nüm-pa $=$ xü je?=k ko?pak-tun-pa-?aj

decir-INCI.I $=\mathrm{EV} \quad$ ese $=\mathrm{AN}$ cabeza-hacer-INCII-I-RLTVZR

204. dizque dice ese, el presidente.

205. yak?ixkeja ya?aj 206. nümpaxü ja?

yak-?üx+kej-a ya?aj nüm-pa=xü ja?

CAUS-encuerar-IMPR éste decir-INCI.I=EV él

205. -iEncuérenlo a éste!-, 206. dizque dice él.

207. yake:ka?a:t ?ituku

yak-ke:k2-a2-a-t $\quad 2 \mathrm{i}=$ tuku

CAUS-quitar-APL2-IMPR-PL.PAH $\quad \mathrm{A} 3$ (POSD)=ropa

207. - Quitenle su ropa!-

208. je? ?u:raxükü ?ijayak?ixkejij

je? $\quad$ iu: $r a=x \ddot{u}=k \quad$ i $i=j a:=y a k-P u ̈ x+k e j-i-j$

ese hora $=\mathrm{EV}=\mathrm{AN} \quad \mathrm{A} 3(\mathrm{ABS})=\mathrm{MIRAT}=\mathrm{CAUS}$-encuerar-INCD-INVD.I

208. A esa bora lo encueraron.

209. je? ?u:raxükü je? ko?paktumpa?aj ?inümi

je? ?u:ra $=x u ̈=k$ je? ko?pak-tun-pa-Paj $\quad$ i $i=n u ̈ m-i$

ese hora $=\mathrm{EV}=\mathrm{AN}$ ese cabeza-hacer-INCI.I-RLTVZR $\mathrm{A3}(\mathrm{ABS})=$ decir-COMD

209. Dizque a esa bora el presidente dice:

210. para ya?aj ja:tu?k miwya:je?ajü mika:jami?na?n

para ya?aj ja:-tuk $\min =w y a: j e-2 a j$

para éste otro-uno $\mathrm{A} 2(\mathrm{POSD})=$ viaje-RLTVZR

$\min =\mathrm{ka}:=\mathrm{ja}:=\operatorname{mi}: \mathrm{n} 2 \mathrm{-a} \mathrm{n}$

$\mathrm{A} 2(\mathrm{ABS})=\mathrm{NEG}=\mathrm{MIRAT}=\mathrm{venir}-\mathrm{IRRD}$

210. -Para que ya no vengas otra vez-, 


$\begin{array}{lll}\text { 211. nümpaxü ja? } & \text { 212. taxnümayu demasya:do } \\ \text { nüm-pa=xü ja? } & \text { tax=nüm-ay-u } & \text { demasya:do } \\ \text { decir-INCI.1=Ev él } & \mathrm{C} 1 \text { (LOCAL)=decir-APL2-COMI } & \text { demasiado } \\ \text { 211. dizque dice él. } 212 . & - \text { Te dije demasiado } & \end{array}$

213. minka:jami?na?n porke si $\min =k a:=j a:=m i=n ?-a ? n \quad$ porke si $\mathrm{A} 2(\mathrm{ABS})=\mathrm{NEG}=\mathrm{MIRAT}=$ venir-IRRD porquesi 213. que no vinieras porque sino

214. miyaktïkiya?xanüp wope mi=yak-tük + li:y?-a?x-an-ü-pa wop-e B2(ABS) $=$ CAUS-entrar-APL2-IRR-INV-INCI.I golpear-NMZR

214. vas a recibir (te van a meter) tu pela (golpes)-,

215. nümpaxü ja? nüm-pa $=x \ddot{u} \quad$ ja? decir-INCI.I=EV él

215. dizque dice él.

216. mü:t jama:k kumpa:ne ?ixiyuk Piya?xi mü:t jamaj=k kumpa:ne $2 \mathrm{ix}+$ ?i:y?-u=k $\quad$ i $=y a: x ?-\mathrm{i}$

y aquel $=\mathrm{AN}$ compadre empezar-COMI $=\mathrm{AN} \mathrm{A} 3(\mathrm{ABS})=$ gritar- $\mathrm{INCD}$

216. Yaquel compadre empezó a gritar

217. Tiyakmo?e wope ?i=yak-mo:y?-e wop-e $\mathrm{A} 3(\mathrm{ABS})=\mathrm{PASV}-\mathrm{dar}-\mathrm{INCD}$ golpear-NMZR

217. cuando le estaban dando de golpes.

218. ju?kupaktükpi je:pak ?i?iti ju?kopak-tük-pi je:-pi=ak $\quad$ i $=$ ?it-i

pueblo-casa-LOC DIST-LOC=AN A3(ABS)=existir-INCD

218. Allá está en el palacio.

$\begin{array}{ll}\text { 219. Pijayak?ixkejij } & \text { 220. nümpaxükü } \\ \text { Pi=ja:=yak-Püx+kej-i-j } & \text { nüm- } \mathrm{pa}=\mathrm{xü}=\mathrm{k} \\ \mathrm{A} 3 \text { (ABS) }=\mathrm{MIRAT}=\mathrm{CAUS}-\mathrm{encuerar-}-\mathrm{NCD}-\mathrm{INVD} . \mathrm{I} & \text { decir-INCI.I=EV=AN } \\ \text { 219. Allá lo estaban encuerando. } 220 . \text { Dizque dice él: }\end{array}$

\begin{tabular}{|c|c|c|}
\hline 221. ?iyakwopi ?oyamej & 222. ?оyа & 223. nümpaxü ja? \\
\hline ?i=yak-wop-i $?$ & Poya & nüm-pa $=x \ddot{u} \quad$ ja? \\
\hline$A 3(A B S)=$ PASV-golpear-COMD bien & bueno & decir-INCI.I=EV él \\
\hline
\end{tabular}


224. je? ?u:raxük ?inümi je? ko?paktumpa?

$$
\begin{aligned}
& \text { je? } \quad \text { u }: r a=x u ̈=k \text { ?i=nüm-i je? ko?pak-tun-pa-? } \\
& \text { ese hora }=\mathrm{EV}=\mathrm{AN} \quad \mathrm{A} 3(\mathrm{ABS})=\text { decir-COMD ese cabeza-hacer-INCI.I-NMZR } \\
& \text { 224. Dizque a esa hora dijo el jefe del pueblo. }
\end{aligned}
$$

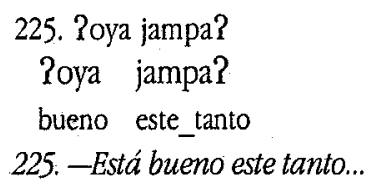

226. tani:wopkitawu?a?k ?ixutu

$$
\begin{aligned}
& \tan =\text { ni:-wop-kitaw- }-\mathrm{a} \mathrm{a}=\mathrm{k} \\
& \mathrm{A1}(\mathrm{ERG})=\text { SUPERF-golpear-revolcarse-COMI-PERF }=\mathrm{AN} \quad \mathrm{A} 3(\mathrm{POSD})=\text { culo } \\
& \text { 226. Ya lo golpé en su culo-, }
\end{aligned}
$$

227. nümpaxü ja? 228. tanwopwa?k seme

$$
\begin{array}{llll}
\text { nüm-pa }=x \ddot{~ j a} & \text { ja? } & \tan =\text { wop-u-Pa } 2=\mathrm{k} & \text { seme } \\
\text { decir-INCI.I }=E \mathrm{EV} & \text { él } & \mathrm{A} 1(\mathrm{ERG})=\text { golpear-COMI-PERF }=\mathrm{AN} \text { muy }
\end{array}
$$

228. dizque dice él. 228. Lo golpé demasiado,
229. machi?tü 230. mü:t ?inükxi 231. jumü ?imini machi?t-ü mü:t $2 i=n u ̈ k x-i \quad$ jumü $2 i=m i n n-i$
soltar-IMPR y $\mathrm{A} 3(\mathrm{ABS})=\mathrm{ir}-\mathrm{INCD}$ donde $\mathrm{A3}(\mathrm{ABS})=$ venir-COMD
229. -iSuéltalo! 230. y que se vaya 231. al lugar de donde vino-,

232. nümpaxü ja? 233. je? ?u:raxükü Tinümi

$$
\begin{aligned}
& \text { nüm-pa=xü ja? je? ?u:ra=xü=k ?i=nüm-i } \\
& \text { decir-INCI. } I=\mathrm{EV} \text { él ese hora }=\mathrm{EV}=\mathrm{AN} \quad \mathrm{A} 3(\mathrm{ABS})=\text { decir- } \mathrm{COMD}
\end{aligned}
$$

232. dizque dice él. 233. A esa bora dijo.

234. ?oya 235. nümpaxü ja?， 236. ?iyü?kne:k tri:ste

$$
\begin{aligned}
& \text { Poya nüm-pa }=x \ddot{u} \text { ja? } \mathrm{i} i=y \ddot{u} 2 \mathrm{k}-\mathrm{nü}-\mathrm{i}=\mathrm{k} \quad \text { tri:ste } \\
& \text { bueno decir-INCIII=EV él } \mathrm{A} 3(\mathrm{ABS})=\text { salir-ya-COMD }=\mathrm{AN} \text { triste }
\end{aligned}
$$

234. -Está bien-, 235. dizque dice él. 236. Ya se salió triste.

237. tristek ?iPiti

$$
\begin{aligned}
& \text { triiste }=\mathrm{k} \quad \mathrm{i}=\mathrm{it}-\mathrm{i} \\
& \text { triste }=\mathrm{AN} \quad \mathrm{A}(\mathrm{ABS})=\text { existir- } \mathrm{N} \mathrm{NCD} \\
& \text { 237. Estaba triste. }
\end{aligned}
$$

238. Riyüiknej tojwopi?i?k

$$
\text { ?i=yü?k-nü-i toj-wop-2i:y?-ik }
$$$$
\mathrm{A} 3(\mathrm{ABS})=\text { salir-ya-COMD APL4-golpear-DIR:adentro-PCP }
$$

238. Salió todo golpeado. 
239. nü:ki?k ?ixutu ?itewa

$$
\begin{array}{ccl}
\text { nü: } \mathrm{k}-\mathrm{ik} & \mathrm{i}=\mathrm{xutu} & \mathrm{P} \mathrm{i}=\text { tewa } \\
\text { mayugarse-PCP } & \mathrm{A} 3(\mathrm{POSD})=\text { culo } & \mathrm{A}(\mathrm{POSD})=\text { nalga } \\
\text { 239. Su nalga estaba mayugada. }
\end{array}
$$

240. jotoyaxük ?ipetnej Rikawa:yu?p

$$
\begin{array}{lll}
\text { jotoya }=\mathrm{xü}=\mathrm{k} & \mathrm{i}=\text { pet-nü- } & \mathrm{i}=\text { kawa:yu-pi } \\
\text { despacio }=\mathrm{EV}=\mathrm{AN} & \mathrm{A} 3(\mathrm{ABS})=\text { subir-ya-COMD } & \mathrm{A} 3(\mathrm{POSD})=\text { caballo-LOC }
\end{array}
$$

240. Ya se subió despacito a su caballo.

241. minu tu?a:p triste

$$
\begin{aligned}
& \text { mi:nP-utuPaw-pi triste } \\
& \text { venir-COMI camino-LOC triste }
\end{aligned}
$$

241. Se vino triste por el camino.

242. ?ijamatnexük Pitükmì

$$
\mathrm{P} i=\text { jamat-nü- } \mathrm{i}=\mathrm{xü}=\mathrm{k} \quad \mathrm{P} \mathrm{i}=\text { tük-mü }
$$$$
\mathrm{A} 3(\mathrm{ABS})=\text { llegar-ya }-\mathrm{COMD}=\mathrm{EV}=\mathrm{AN} \quad \mathrm{A} 3(\mathrm{POSD})=\mathrm{casa}-\mathrm{LOC}
$$

242. Ya cuando llegó en su casa.

$$
\begin{array}{cl}
\text { 243. nümpaxük Pimajaw } & \text { 244. minto:ku } \\
\text { nüm-pa }=x \ddot{u}=\mathrm{k} \quad \mathrm{P}=\text { majaw } & \mathrm{min}=\text { to:k-u } \\
\text { decir-INCI.I=EV=AN } \mathrm{A} 3(\mathrm{POSD})=\text { mujer } & \mathrm{A} 2(\mathrm{ERG})=\text { vender-COMI }
\end{array}
$$

243. Dizque dice su mujer: 244. - ila vendiste?-

$\begin{array}{ll}\text { 245. mas ke wope tyakmoyi } & \text { 246. nümpaxü ja? } \\ \text { mas ke wop-e tan=yak-mo:y2-i } & \text { nüm-pa=xü ja? } \\ \text { más que golpear-NMZR A1(ABS)=PASV-dar-COMD } & \text { decir-INCl.I=EV él } \\ \text { 245. Me dieron de puros golpes-, 246. dizque dice él. } & \end{array}$

247. ya?k kumpa:ne yak?a:wixnüp te:ke?

$$
\begin{array}{lll}
\text { ya?aj=ak kumpa:ne } & \text { yak-?a:wix-nü-pa } & \text { te } ?=\mathrm{k}=\mathrm{je} ? \\
\text { este=AN compadre } & \text { PASV-esperar-ya-INCI.I de_verdad=AN=ese }
\end{array}
$$

247. -Si ya lo estaban esperando a este compadre-,

248. nümpaxui ja? 249. posü Toya 250. nümpaxü ja? nüm-pa $=x u ̈$ ja? pos ?oya nüm-pa $=x \ddot{u}$ ja? decir-INCLII=EV él pues bueno decir-INCIII=EV él

248. dizque dice él. 249. -Pues está bueno-, 250. dizque dice él.

251. jemxü?ü Tikumpa:ne jem-xü ?i=kumpa:ne alli $\mathrm{EV} \quad \mathrm{A} 3(\mathrm{POSD})=$ compadre

251. Dizque abi su compadre, 
252. jamaj ja:tukaj ?ikumpa:ne ?iyakju:niyuk ta?na

$$
\text { jamaj ja:-tuk-?aj } \quad \text { ii=kumpa:ne }
$$

aquel otro-uno-RLTVZR $\mathrm{A} 3(\mathrm{POSD})=$ compadre

?i=yak-ju:n-ni:y?-u=k ta?na

$\mathrm{A} 3(\mathrm{ERG})=$ CAUS-sentar-DIR:sin_mov-COMI=AN mucho

252. el otro su compadre, ganó más

253. porke je? ko?yaj moyüwak me:nyu

porke je? ko?yaj mo:y?-ü-w=ak me:nyu

porque ese diablo dar-INV-COMI=AN dinero

253. porque el diablo le dió el dinero.

254. mü:tak ya?aj ?emwe:sak ?iyakju:ni?am

mü:t=ak ya?aj ?en-we:s=ak ?i=yak-ju:n-ni:y?-am

$\mathrm{y}=\mathrm{AN} \quad$ éste en-vez=ANA3(ERG) $=$ CAUS-sentar-DIR:sin_mov-IRRI

254. Yéste (el compadre que no recibió dinero) en vez de que gane

255. tato:ka?n tü?ni

$$
\text { ta }=\text { to:k-a?n tü:n?-i }
$$

$\mathrm{C} 3(\mathrm{ERG})=$ vender-IRRD $\quad$ cagar-NMZR

255. al vender caca,

256. mejorak ?iyakni:wopkitawnej

mejor=ak Pi=yak-ni:-wop-kitaw-nü-i-j

mejor $=\mathrm{AN} \quad \mathrm{A} 3(\mathrm{ABS})=$ PASV-SUPERF-golpear-revolcarse-ya-INCD-INVD.I

256. mejor le dieron de golpes.

257. nümpaxükü 258. Roya 259. nümpaxü ja?

nüm-pa $=x \ddot{u}=\mathrm{k} \quad$ ?oya nüm-pa $=x \ddot{u} \quad$ ja?

decir-INCLII $=\mathrm{EV}=\mathrm{AN}$ bueno decir-INCI.I=EV él

257. Dizque dice él: 258. -Está bueno-, 259. dizque dice él.

260. dejem ?itiji je? kumpa:ne

de-jem $\quad \mathrm{i}=$ tij-i $\quad$ je? kumpa:ne

de-allí $\quad A 3(A B S)=q u e d a r-C O M D$ ese compadre

260. De abi (asi) se quedó ese compadre,

261. xokwa?koj ?itü:ya?mpi

xok-w-a?=koj $\quad \mathrm{P} i=t u ̈: y-a n-p i$

acostarse-COMI-RLTVZR=nomás $\quad$ A3(POSD) $=$ colgar-NMZR_INSTR-LOC

261. el que nomás estaba acostado en su bamaca. 
Referencias

BAKER, Mark

1988 Incorporation. Chicago: Chicago University Press.

1996 The Polysynthesis Parameter. Oxford: Oxford University Press.

CALDERÓN, Eustorgio

1908 Estudios lingüísticos, vol 2: Las lenguas de Oluta, Sayula, Texistepec, en el Istmo de Tebuantepec en México. Guatemala.

CAMPBelL, Lyle, Terrence S. Kaufman, Y Thomas Smith-STARK

1986 "Meso-America as a Linguistic Area", Language 62: 530-570.

CAMPBell, Lyle y Martha C. MunTzel

1989 "The structural consequences of language death", en Investigating obsolescence. Studies in Language Contraction and Death, Nancy C. Dorian, editora, pp.181- 196. Cambridge: Cambridge University Press.

CLARK, Lawrence E.

1981 Diccionario popoluca de Oluta. México: Instituto Lingüístico de Verano.

1981 Sayula Popoluca Verb Derivation. Dallas: Summer Institute of Linguistics.

COMRIE, Bernard

1981 Language Universals and Linguistic Typology. Oxford: Basil Blackwell.

DeLANCEY, Scott

1981 "An interpretation of split ergativity and related patterns", Language 51.3:626-57.

1997 "Mirativity: The grammatical marking of unexpected information", Linguistic Typology 1:1:33-52.

DIXON, R. M. W.

1994 Ergativity. Cambridge: Cambridge University Press.

DRYER, Mathew

1986 "Primary objects, secondary objects, and antidative", Language 62:808845.

1991 "Subject and inverse in Kutenai", en Papers from the American Indian Languages Conference, UC Santa Cruz, Occasional Papers in Linguistics, J. Redden, editor. Vol. 16, pp.183-192. Carbondale, Ill.: University of Southern Illinois.

1994 "The discourse function of the Kutenai inverse", en Voice and Inversion, T. Givón, editor, pp.65-99. Amsterdam: John Benjamins.

1997 "On the six-way word order typology", Studies in Language 21:69-103. 
DuBors, John A.

1985 "The discourse basis of ergativity", Language 64:805-855.

FOLEY, William A. Y Robert D. VAN VALIN

1985 "Information packaging in the clause", en Language Typology and Syntactic Description I. Clause structure, T. Shopen, editor, pp.282-364. Cambridge: Cambridge University Press.

FoRIs, David

1993 A Grammar of Socbiapan Cbinantec. Tesis doctoral, University of Auckland, Nueva Zealandia.

GRINEVALD CRaIG, Colette

1997 "Language contact and language degeneration", en The Handbook of Sociolinguistics, F, Coulmas, editor, pp,257-270. Oxford: Blackweil Publishers.

HOCKETT, Charles

1966 "What Algonquian is really like", International Journal of American Linguistics 32:59-73.

Grón, Talmy

1994 "The pragmatics of de-transitive voice: Functional and typological aspects of inversion", en Voice and Inversion, T. Givón, editor, pp.3-45. Amsterdam: John Benjamins.

Grvón, Talmy, editor

1994 Voice and Inversion. Amsterdam: John Benjamins.

KaUfman, Terrence $S$.

1995 Mije-Sokean Comparative Grammar. Pittsburgh: University of Pittsburgh MS.

KLAIMAN, M. H.

1989 "Inverse voice and head-marking in Tanoan languages", en Chicago Linguistic Society 25/1, C. Wiltshire et al., editores, pp. 258-271. Chicago: Chicago Linguistic Society.

1992 "Inverse Languages" Lingua 88: 227-261.

1993 "The Relationship of Inverse Voice and Head-marking in Arizona Tewa and Other Tanoan Languages", Studies in Language 17.2:343-370.

LEHMANN, Walter

1920 Zentral-Amerika. Vol II. Berlin: Verlag Dietrich Reimer (Ernst Vohsen).

LEVY, Paulette

1994 How sbape becomes grammar. On the semantics of part morphemes in Totonac. Working Paper 29. Cognitive Anthropology Research Group, Max Planck Institute for Psycholinguistics. Nijmegen, Holanda.

MITHUN, Marianne

1984 "The evolution of noun incorporation", Language 60: 847-95. 
NicHols, Johanna

1986 "Head-marking and dependent-marking grammar", Language 62.1:56119.

PAYNE, Doris L.

1993 "Nonconfigurationality and discontinuous expressions in Panare", en Proceedings of the Nineteenth Annual Meeting of the Berkeley Linguistics Society. Special Session on Syntactic Issues in Native American Languages, David A. Peterson, editor, pp.121-138. Berkeley: Berkeley Linguistics Society.

1994 "The Tupí-Guaraní inverse", en Voice Form and Function, Barbara Fox y Paul Hopper, editores, pp.313-340. Amsterdam: John Benjamins.

PAYNE, Doris L., Hamaya, MITSUYO, Y Peter JACOBS

1994 "Active, Inverse and Passive in Masai", en Voice and Inversion, T. Givón, editor, pp.283-315. Amsterdam: John Benjamins.

THOMPSON, Chad

1994 "Passive and inverse constructions", en Voice and Inversion, T. Givón, editor, pp.47-63. Amsterdam: John Benjamins.

WICHMANN, Søren

1993 "Grammaticalization in Mixe-Zoquean languages", Spracblvpologie und Universalienforschung 46:45-60.

1995 The relationship among the Mixe-Zoquean Languages of Mexico. Salt Lake City: University of Utah Press.

WILKINS, David P.

1991 "The Semantics, Pragmatics and Diachronic Development of 'Associated Motion' in Mparntwe Arrernte", Buffalo Papers in Linguistics 1991, 1:207257.

ZAVALA, Roberto

1994 "Inverse alignment in Huastec", Función 15-16: $27-81$.

1998a "External Possessor in Oluta Popoluca (Mixean): Applicatives and Incorporation of Relational Terms", en External Possessor, Noun Incorporation and Related Constructions, D. Payne y L. Barshi, editores, pp. 339-372. Amsterdam: John Benjamins.

1998b "Oluta Popoluca Trilingual Dictionary". Nijmegen: Max Planck Institute MS. 
The three Olutec stories from Oluta, Veracruz, were narrated by Antonio Asistente Maldonado. Roberto Zavala presents a morpheme-by-morpheme analysis of the texts with a sketch of the major grammatical and typological features of this language. Olutec is spoken by three dozen speakers. The grammatical structure of this language has not been described before. The sketch contains information on verb and noun morphology, verb classes, clause types, inverse/direct patterns, grammaticalization processes, applicatives, incorporation, word order type, and discontinuous expressions. The stories presented here are the first Olutec texts ever published. The motifs of the stories are well known throughout Middle America. The story of "the Rabbit who wants to be big" explains why one of the main protagonists of Middle American folktales acquired long ears. The story of "the Devil who is inebriated by the people of a village" explains how the inhabitants of a village discover the true identity of a man who likes to dance buapango and decide to get rid of him. Finally the story of "the shit-sellers" presents two compadres, one who is lazy and the other one who works hard. The hard-worker asks the lazy compadre how he survives without working. The latter lies to to him that he sells shit in the neighboring village. The hard-working compadre decides to become a shit-seller and in the process realizes that the lazy compadre deceived him. However, he is lucky and meets with the Devil who offers him money in compensation for having been deceived. When the lazy compadre realizes that the hard-working compadre has become rich, he tries to do the same business but gets beaten in the process. 